\title{
AACR/SNMMI State-of-the-Art Molecular Imaging in Cancer Biology and Therapy: Abstracts
}

Exciting developments in the field of cancer imaging have led to important insights into the origins and biology of cancer, as well as novel techniques for early diagnosis, accurate staging, and monitoring of response to therapy for cancer patients. These new discoveries have great potential as tools for basic and translational research in cancer biology and for clinical use to improve patient outcomes.

The Society of Nuclear Medicine and Molecular Imaging (SNMMI) and the American Association for Cancer Research (AACR) have come together to cosponsor State-of-the-Art Molecular Imaging in Cancer Biology and Therapy, a conference to be held February 27 to March 2, 2013 , at the Manchester Grand Hyatt in San Diego, California. The focus of the conference will be to educate, promote, and support the application and use of molecular imaging in cancer biology. The conference will cover molecular imaging and therapy in cancer-related basic, translational, and clinical research and the integration and application of molecular imaging to the understanding of cancer biology. The goal of this joint AACRSNMMI conference is to bring imaging scientists together with basic, translational, and clinical cancer researchers in a focused, smaller meeting setting to discuss the latest developments in imaging genetic and molecular processes of cancer as they occur in cells, animal models, and patients.

The conference will feature several concurrent educational sessions on Wednesday, including "Imaging Therapeutic Response with PET," "Emerging Technologies," and "In Vivo Studies of Cancer Biology at Cellular Resolution." Wednesday evening will include the welcome reception and a special keynote address from Douglas Hanahan, PhD, Professor of Molecular Oncology and Merck Serono Oncology Chair at the École Polytechnique Fédérale de Lausanne and Director of the Swiss Institute for Experimental Cancer Research. Thursday and Friday will feature 8 plenary sessions focused on cancer stem cells; imaging of the tumor microenvironment, stroma, and metastatic niche; imaging and genomics; innovative therapies guided by imaging; and more.

This conference will also offer opportunities for participation from junior scientists, with presentations selected from the most highly rated abstracts. In addition to the didactic lectures, the meeting will also include over 90 poster presentations. The abstracts presented in this supplement issue to The Journal of Nuclear Medicine represent some of the most interesting ideas in molecular imaging research as applied to cancer biology and therapy. The abstracts are listed alphabetically by the last name of the presenting author, with the main abstracts followed by the invited speaker abstracts. Please note that the presenting author at the conference is not always the first author of the abstract. In addition, abstracts selected to receive Young Investigator Travel Awards (AACR-Aflac, Incorporated, Scholar-in-Training Awards and SNMMI/AACR Young Investigator Awards) are indicated by a footnote.

We hope that many of our members will be able to attend this exciting conference.

Carolyn J. Anderson, PhD Cochair, State-of-the-Art Molecular Imaging in Cancer Biology and Therapy; and Professor of Radiology and Director of the Molecular Imaging Laboratory,

University of Pittsburgh

David Piwnica-Worms, MD, PhD

Cochair, State-of-the-Art Molecular Imaging in Cancer Biology and Therapy; and Professor of Radiology, Professor of Cell Biology and Physiology,

Director of the Molecular Imaging Center, and Director of the BRIGHT Institute,

Washington University in St. Louis

\section{Abstract Reviewers}

David Piwnica-Worms, MD, PhD

Washington University School of Medicine, St. Louis, MO

Carolyn J. Anderson, $\mathrm{PhD}$

University of Pittsburgh Medical Center, Pittsburgh, PA

Christopher Contag, PhD

Stanford University, Stanford, CA

Kim Kelly, PhD

University of Virginia, Charlottesville, VA

Hisataka Kobayashi, MD, PhD

National Cancer Institute, Bethesda, MD
Steve Larson, MD, FACNM

Memorial Sloan-Kettering Cancer Center, New York, NY

Jason Lewis, $\mathrm{PhD}$

Memorial Sloan-Kettering Cancer Center, New York, NY

Martin Pomper, MD, PhD

Johns Hopkins Kimmel Comprehensive Cancer Center, Baltimore, $M D$

Zena Werb, PhD

University of California, San Francisco, CA

COPYRIGHT @ 2013 by the Society of Nuclear Medicine and Molecular Imaging, Inc.

DOI: $10.2967 /$ jnm542abs 


\section{MAIN ABSTRACTS}

1

Image-guided treatment of pancreatic cancer using cathepsin E-activatable prodrug. W.R. Abd-Elgaliel ${ }^{1}$, Z. Cruz-Monserrate ${ }^{2}, \mathrm{H}$. Wang $^{2}$, C.D. Logsdon ${ }^{2}$, C. Tung ${ }^{1} ;{ }^{1}$ The Methodist Hospital Research Institute-Weill Cornell Medical College, Houston, TX, ${ }^{2}$ The University of Texas MD Anderson Cancer Center, Houston, TX

Purpose: Upregulated cathepsin E (Cath E) protease has been found associated with pancreatic cancer. Here we assessed the competence of pancreatic cancer-associated Cath $\mathrm{E}$ as a drug activator for non-invasive imaging and treatment of pancreatic cancer in mice. Specific activation of the prodrug is expected to kill pancreatic cancer cells without harming normal pancreatic cells. Procedures: A Cath E-activatable prodrug and imaging probe was synthesized and evaluated for its enzyme susceptibility and selectivity. Fluorescence imaging of pancreatic cancer was validated using cells and animals with different expression of Cath $\mathrm{E}$ activity. Potential of laser treatment was assessed by detecting the intracellularly activated prodrug in Cath E-positive cancer cells (Mpanc96-CTSE). The conversion of the drugs was monitored by newly formed fluorescent signal and their phototoxicity was demonstrated by applying specific laser. Negative controls were assessed in parallel with low Cath E expressing cells. Data: Non-invasive imaging of pancreatic cancer was achieved using a Cath E-sensitive prodrug. Images illustrate the capability of detecting the fluorescence within Cath E-positive tumors but not within the normal pancreas. Treatment results indicate that prodrug was activated specifically within Cath E-positive tumors. No signs of prodrug activation in the normal pancreatic tissues were detected. When used in combination with light illumination, it shows selective therapeutic effect to the cancerous tissues, with minimal harm to the adjacent normal tissues. Conclusions: The novel Cath E-mediated prodrug activation approach allows the detection of pancreatic cancer cells accurately and specifically impairs their viability. Most importantly, it does not harm the adjacent normal pancreatic cells. The treatment experiments substantiate the usefulness of site-specific endogenous activation of Cath E-sensitive prodrug for pancreatic cancer treatments. The study demonstrates the potential of applying Cath E-activatable prodrug with laser in treating Cath E-rich pancreatic cancer. This treatment approach could result in fewer side effects than the nonspecific treatments currently in use.

\section{2}

Antibody-CpG conjugate for immunotherapy using NY-ESO-1 intracellular tumor-associated antigen target. D.S. Abou, T. Noguchi, G. Ritter, J.S. Lewis; Memorial Sloan-Kettering Cancer Center, New York, NY

NY-ESO-1 is an intracellular tumor antigen expressed in various tumor cells but absent of somatic cells except in testicular germ cells. Thus, NY-ESO-1 is an interesting target for cancer treatment. We have shown that the combination treatment of anti-NY-ESO-1 monoclonal mouse antibody $(\mathrm{mAb})$ such as clone E978 and chemotherapy such as 5-FU led to an anti-tumor effect (1). The immune complex of E978 and NY-ESO-1 was formed locally on tumor sites only when E978 was administered with 5-FU which accelerated the release of intracellular tumor antigen. The passively formed immune complex elicited augmented dendritic cells (DC) maturations and NY-ESO-1 specific CD8+ T cells. This therapeutic strategy efficiently inhibited tumor growth; however, it rarely achieved complete tumor regression. Recently, a synthetic single DNA strand CpG-ODN has been identified and clinically tested for cancer treatment. CpG-ODN is recognized by TLR-9 (Toll-Like Receptor 9) located on endosomes and cytosols. CpG-ODN recognition invokes cytotoxic immune responses against tumor cells. Since $\mathrm{CpG}$ is relatively toxic, it was required to intratumorally administer the agent in order to optimize its therapeutic effect (2). To specifically deliver CpGODN on tumor sites and synergistically enhance the anti-tumor effect of E978 and CpG, we focused on combining E978 antibody with CpG-ODN. An immuno construct E978-CpG was synthesized and the therapeutic effect of the novel conjugate was tested. $\mathrm{CpG}$ was conjugated to E978 using a

*AACR-Aflac, Incorporated, Scholar-in-Training Award winner. bifunctionalized isothiocyanate spacer and the construct was chemically characterized using size exclusion chromatography and electrophoresis. The immunoconjugate was biologically tested using ELISA test to verify the immuno affinity with NY-ESO-1. Furthermore, E978-CpG conjugate was in vitro and in vivo evaluated. After $\mathrm{CpG}$ modification, $\mathrm{E} 978-\mathrm{CpG}$ conjugate was allowed to be formed overnight incubated at room temperature. The construct was separated from unreacted DNA using size exclusion chromatography. The electrophoresis confirmed the conjugation efficiency, showing an additional heavier band (higher molecular weight) for the new construct as compared to E978. ELISA test demonstrated a minimum immunological action change of modified E978 as compared to E978. This construct responded to NY-ESO-1 test with a comparable affinity to that of E978. Furthermore, the in vitro evaluation on DC indicated a dramatical enhancement of cell maturation with CpG-E978, 70\% of DCs were observed maturated as compared to only $10 \%$ for E978. Furthermore, CpGE978 was comparatively evaluated on DC maturation with $\mathrm{CpG}$ alone in order to define the number of DNA strand per antibody. It was found that one antibody was conjugated to one $\mathrm{CpG}$. Finally, the modified antibody E978 was evaluated in vivo using tumor bearing mice (BALB/c mice xenografted with CT26, NY-ESO-1 expressing cells). A comparative study with 6 animal groups was conducted looking at the tumor growth over 25 days with: no treatment; 5-FU treatment; 5-FU + E978; 5-FU + E978-CpG; 5-FU + A33 as a control antibody, 5-FU + A33-CpG. As expected our positive control group, 5-FU + E978, showed significant inhibition of tumor growth as compared to 5-FU and the A33 control groups. However, no augmented tumor growth inhibition was seen when treating the mice with 5-FU + E978-CpG as compared to 5-FU + E978. E978-CpG was successfully synthesized and characterized, validating the conjugation strategy. The novel antibody construct showed high immune response to DC maturation. DC maturation was 7 times higher when treated with our construct as compared to control. The results were seen to be very promising in vitro, demonstrating the possible synergistic therapeutic and targeting effect of $\mathrm{CpG}$ associated with E978, however, the inhibition of tumor growth was limited in vivo. A possible difficulty may have risen regarding the amount of antibody construct delivered on the tumor site, due to possible different pharmacokinetics between E978 and E978-CpG. References: 1. Noguchi T et al., Cancer Research. 2012 Apr 1;72(7):1672-82. 2. Karbach J et al., Cancer Research. 2012 Sep 1;72(17):4304-10.

\section{3*}

PET tracking of ${ }^{89} \mathrm{Zr}$-radiolabeled immune cells. D.S. Abou, D.L.J. Thorek, K. Sevak, A.R. Slamowitz, J.S. Lewis; Memorial Sloan-Kettering Cancer Center, New York, NY

Non-invasive in vivo tracking of cells provides insight into the temporal dynamics of cell populations in the context of disease or inflammation. Cell tracking has been previously demonstrated with iron oxide nanoparticles for MRI, or ${ }^{111} \mathrm{In}$-oxine and ${ }^{99 \mathrm{~m}} \mathrm{Tc}$-colloid radiolabeled cells for sensitive Single-Photon Emission Computed Tomography (SPECT) detection. These approaches suffer from the limitations inherent in each modality; MRI has reduced sensitivity while SPECT tracer quantification is often difficult. The utilization of a Positron Emission Tomography (PET) approach would allow whole body imaging as well as precise and sensitive quantification of cell distribution. Attempts to date have been hindered by the mismatch of cell distribution kinetics and too-short radiotracer half-life (e.g. ${ }^{18} \mathrm{~F} \mathrm{t}_{1 / 2}=109.7$ $\mathrm{min}$ ). Towards this goal we have developed a novel macrophage (MP) and immune cell labeling methodology utilizing stable ${ }^{89} \mathrm{Zr}$-phosphate microparticles $\left({ }^{89} \mathrm{Zr} \mathrm{t}_{1 / 2}=78.4 \mathrm{~h}\right)$ for PET/CT imaging. Two approaches were investigated to radiolabel cells: 1) MP (RAW 264.7) were ex vivo radiolabeled using ${ }^{89} \mathrm{Zr}$-phosphate microcolloid and intravenously administered to mice, and 2) ${ }^{89} \mathrm{Zr}$-phosphate microcolloids were directly intravenously injected in mice to in vivo radiolabel the activated immune cell population. Cells were then imaged longitudinally in healthy mice (NCRNU-M; female) and mice bearing either inflammation or xenograft models. In the ex vivo radiolabeling approach, labeling resulted in high cell viability (90\%) and a labeling efficiency of $45 \%$ of initial dose (ID) with $120 \mu \mathrm{Ci}$ for $1 \times 10^{6}$ cells. $24 \mathrm{~h}$ post-cell harvesting, $85 \%$ of the ID remained associated with MPs. Since minimal uptake was observed at $4^{\circ} \mathrm{C}$, particle internalization was temperature dependent, demonstrating an energydependent uptake mechanism related to phagocytotic activity. ${ }^{89} \mathrm{Zr}$-labeled cells were injected in healthy mice and tracked longitudinally using PET. At 
1 and $4 \mathrm{~h}$ post-injection (p.i.) the majority of the cells accumulated in: lungs $\left(\% \mathrm{ID} / \mathrm{g}_{\max }: 38.6 \pm 2 ; 22.0 \pm 8\right)$, spleen $\left(\% \mathrm{ID} / \mathrm{g}_{\max }: 15.0 \pm 1.8 ; 20.9 \pm 1\right)$ and liver $\left(\% \mathrm{ID} / \mathrm{g}_{\max }: 36.0 \pm 1.7 ; 43.0 \pm 3\right)$. After $24 \mathrm{~h}$ the activity in lungs cleared $\left(<10 \% \mathrm{ID} / \mathrm{g}_{\text {max }}\right)$ while the activity in liver $(>50 \% \mathrm{ID} / \mathrm{g})$ and spleen $(>20 \% \mathrm{ID} / \mathrm{g})$ increased. Bone uptake was negligible $\left(<2 \% \mathrm{ID} / \mathrm{g}_{\max }\right)$, even at late time points. This demonstrates negligible leakage of ${ }^{89} \mathrm{Zr}$ from the MPs. Further investigations are underway to monitor ex vivo labeled cells in inflammation and tumor models. Direct administration of ${ }^{89} \mathrm{Zr}$-microcolloid was conducted in healthy mice. At 1 and $4 \mathrm{~h}$ p.i, the liver and spleen presented a characteristically high radioactive uptake (respectively $>30 \% \mathrm{ID} / \mathrm{g}$ and $\sim 20 \% \mathrm{ID} / \mathrm{g}$ ) associated with particle pharmacokinetics. The blood and bone radioactivity were observed to be similar $(2-3 \% \mathrm{ID} / \mathrm{g})$ and the harvested bone marrows presented $5-10 \%$ of the total bone radioactivity. This was seen to decrease over time, which is a contrary observation for a ${ }^{89} \mathrm{Zr}$-radiotracer (a bone-seeker). Further investigations are in progress to determine the quality of cells radiolabeled and whether this tool could be used to discriminate inflammation from tumor. We have successfully developed a novel technique to radiolabel MPs with ${ }^{89} \mathrm{Zr}$-phosphate microcolloids for longitudinal, quantitative and whole-body imaging. Ex vivo radiolabeled MPs show low bone uptake and active migration of radioactivity. Direct administration of ${ }^{89} \mathrm{Zr}$-microcolloids resulted in a pharmacodynamic profile similar to ${ }^{99 \mathrm{~m}} \mathrm{Tc}$-colloid (1). PET radiolabeled MP or immune cells are under further investigation to gain insight into MP recruitment and host response at sites of inflammation and tumors. Reference: 1. H.L. Atkins et al. J Nucl Med., 1968, (9), 163-164.

\section{4}

Whole-body diffusion MRI: a quantifiable technique to improve lesion detection, tumor staging, and assess response to therapy. $\mathrm{R}$. Attariwala, W. Picker; AIM Medical Imaging, Vancouver, BC, Canada

Technical advances in magnetic resonance imaging (MRI) have recently permitted the ability to scan the whole body in one sitting. These new developments permit the application of multiple sequences to interrogate tissue composition with both high spatial and contrast resolution, resulting in improved lesion detection and characterization. Until recently, body applications of MRI were limited to conventional morphologic techniques which are used to assess tissue composition, with intravenous gadolinium aiding in assessing increased tissue vascularity or permeability. Functional MRI techniques such as diffusion weighted imaging (DWI) have until recently been limited to assessment of the stationary central nervous system. Diffusion weighted imaging with background signal suppression (DWIBS) is a functional MRI technique based on water motion with subtraction of the fat signal contribution from body tissue. This technique is able to assess changes in the tissue microenvironment at a cellular level. With modern MRI hardware, this functional imaging technique can be applied to image the whole body with a free breathing technique. Lesions which demonstrate 1) an increase in intra or extracellular tissue packing density, and thus less water Brownian motion, or 2) fluid that has increased viscosity, yield an increase in signal which results in increased target signal relative to background tissue. The degree of diffusion or free water motion can be quantified as a machine independent parameter. DWIBS coupled with conventional whole body MRI sequences at 1.5 Tesla can been used to not only detect, but help with evaluation of systemic malignant conditions, such as lymphoma, bony metastases and multiple myeloma. It can aid in characterizing lesions in the chest, abdomen and pelvis. DWI also has the benefit of being able to separate out simple ascites from peritoneal carcinomatosis by utilizing the property of differences in viscosity between the two fluids. DWIBS has the ability to aid in assessment of response to therapy by quantifying the change in free bulk water movement at the cellular level; thus being an indirect measure of cell lysis. This technique when applied to the whole body can also be used to quantify changes in whole body tumor burden, without the need for intravenous gadolinium contrast administration. Advanced imaging techniques have shown that combining both anatomic and functional imaging techniques together yields a powerful imaging combination that results in improved lesion detection, characterization, and quantification. An overview of the theory and applications of DWIBS coregistered to conventional whole body MRI sequences will be presented.
Imaging sunitinib-induced vascular regression. Y. Kato ${ }^{1}$, W. Zhu ${ }^{1}$, D. Artemov $^{1}$, S.K. Sarkar ${ }^{2}$, M.V. Backer ${ }^{3}$, J.M. Backer ${ }^{3} ;{ }^{1}$ Johns Hopkins University, Baltimore, MD, ${ }^{2}$ Sanofi Oncology, Cambridge, MA, ${ }^{3}$ SibTech, Inc., Brookfield, CT

Vascular endothelial growth factor (VEGF) acting via corresponding receptors (VEGFR) promotes and supports tumor angiogenesis. VEGF/ VEGFR signaling is the major target for current approved and experimental anti-angiogenic drugs. As reported in preclinical and several clinical studies, at the beginning of the treatment these drugs induce transient vascular regression followed by vascular rebound. These effects are detectable by immunochemical analysis of endothelial markers and by molecular imaging of endothelial markers with targeted PET and SPECT tracers $(1,2)$. The underlying mechanisms of drug-induced transient vascular regression and ultimate rebound are not established. One possibility would be a shedding of drug-affected endothelial cells into the circulation. Alternatively, it is possible that drug-affected endothelial cells are "consumed" locally by tumorassociated macrophages. We tested these alternatives in orthotopic breast cancer model, using MDA231luc (a luciferase-expressing derivative of MDA-MB-231 cells) human breast carcinoma grown in immunodeficient mice. Prior to sunitinib treatment $(80 \mathrm{mg} / \mathrm{kg}$ per day for 4 days, orally) tumor endothelial cells were tagged with a near-infrared fluorescent tracer, $\mathrm{scVEGF/Cy}$. This tracer is a monomeric protein combining two receptorbinding 3-112aa fragments of VEGF site-specifically derivatized with Cy5.5 fluorescent dye via an $\mathrm{N}$-terminal cysteine-containing tag (3). In orthotopic MDA231luc model, scVEGF/Cy accumulates predominantly in tumor endothelial cells via VEGFR-mediated endocytosis and allows for a long-term in vivo near-infrared fluorescent imaging of the tumor vasculature. In the course of sunitinib therapy, mice were daily imaged, and intensities of the signal in the areas of interest in individual mice were determined for each time point. As a result of sunitinib therapy, the signal intensity in all control and all but one treated mice declined approximately 3 -fold. The kinetics of the signal decline were approximated as exponential and half-life of Cy5.5 in tumors was calculated for individual mice. There was no difference between control and treated cohorts, suggesting that the clearance of Cy5.5 from the vasculature of control and sunitinib-treated mice is determined by similar mechanism(s), and that there is no additional sunitinib-induced mechanism(s), such as physical loss of endothelial cells. In vivo imaging data are in sharp contrast with immunohistochemical analysis of tumor tissue obtained from those same mice: a dramatic decline in the prevalence of pan-endothelial marker CD31 and VEGFR-2 in treated mice indicates significant sunitinib-induced vascular regression. Similarly, imaging VEGFR-2 with targeted SPECT tracer indicates significantly lower tracer uptake after 5-day sunitinib treatment (2). Comparing imaging and immunohistochemical data, it is tempting to suggest that sunitinib-induced vascular regression is due to the rapid local "consumption" of endothelial cells together with internalized Cy 5.5 by tumor-associated macrophages, rather than to a physical loss of endothelial cells. Furthermore, it appears that pre-tagging endothelial cells with imaging tracers via VEGF receptors and imaging VEGF receptors in the course of therapy can provide different and complementary information on anti-angiogenic therapy responses. References: 1 . Blankenberg et al. Transl Oncol. 3(1):56-64, 2010. 2. Levashova et al. J Nucl Med. 51(6):959-66, 2010. 3. Backer et al. Nature Med. 13(4):504-9, 2007.

\section{6}

$\left[{ }^{89} \mathrm{Zr}\right]$ bevacizumab PET scan in NSCLC patients. I. Bahce, M.C. Huisman, E. Verwer, R.C. Schuit, D.J. Vugts, G.A.M.S. van Dongen, R. Boellaard, E.F. Smit; VU University Medical Center, Amsterdam, The Netherlands

Rationale: Bevacizumab, an antibody against vascular endothelial growth factor (VEGF), when added to carboplatin-paclitaxel in non-small cell lung cancer (NSCLC) patients, provides a survival advantage over chemotherapy alone. Imaging in vivo tumor bevacizumab pharmacokinetics using PET may be a predictive marker. To assess whether the uptake of radiolabeled bevacizumab, $\left[{ }^{89} \mathrm{Zr}\right]$ bevacizumab $\left(\left[{ }^{89} \mathrm{Zr}\right]\right.$ beva), could be visualized and quantified in NSCLC tumors using PET, we conducted a pilot study, the first in NSCLC patients. Methods: We included 8 patients with advanced NSCLC. All patients underwent an $\left[{ }^{18} \mathrm{~F}\right]$ FDG-PET/CT scan. Next, $36.4 \pm 0.9 \mathrm{MBq}\left[{ }^{89} \mathrm{Zr}\right]$ beva was injected intravenously, followed by $10-\mathrm{min}$ 
PET/CT scans after 4 and 7 days, respectively. For each PET/CT scan, volumes of interest (VOI) were drawn manually in tumor tissue, lymph node metastases, aorta and non-tumor tissue (muscle, healthy lung, fatty tissue) on low-dose CT scans. Subsequently, these VOI were projected onto the PET scans to calculate $\mathrm{SUV}_{\text {mean }}$ and $\mathrm{SUV}_{\text {max }}$ for each VOI. Therefore, SUV parameters refer to the anatomically defined whole of the tumor tissue Results: For all tumors, $\left[{ }^{89} \mathrm{Zr}\right]$ beva uptake was heterogeneously distributed within tumor tissue, showing highest uptake in the rims of the tumors (in 6 out of 8 patients) on visual assessment. Tumor SUV values were higher than non-tumor tissue on days 4 and $7(\mathrm{P}<0.01$ on days 4 and 7$)$. Tumor $\left[{ }^{89} \mathrm{Zr}\right]$ beva $\mathrm{SUV}_{\text {mean }}$ correlated significantly with $\left[{ }^{18} \mathrm{~F}\right] \mathrm{FDG} \mathrm{SUV}$ mean $(\mathrm{P}<0.01$ on days 4 and 7), whereas $\left[{ }^{89} \mathrm{Zr}\right]$ beva $\mathrm{SUV}_{\max }$ only correlated significantly with $\left[{ }^{18} \mathrm{~F}\right]$ FDG SUV ${ }_{\max }$ on day $7(\mathrm{P}=0.06$ and $\mathrm{P}=0.02$ on days 4 and 7 , respectively). Lymph node metastases showed no significant correlation with $\left[{ }^{18} \mathrm{~F}\right]$ FDG. Conclusion: $\left[{ }^{89} \mathrm{Zr}\right]$ beva shows high tumor-to-background ratios, allowing visualization and quantification of uptake in tumors using simplified parameters. Visually, tumor $\left[{ }^{89} \mathrm{Zr}\right]$ beva uptake appears heterogeneous with maximum uptake located in the rims of tumor tissue $\left[{ }^{89} \mathrm{Zr}\right]$ beva correlated with $\left[{ }^{18} \mathrm{~F}\right] \mathrm{FDG}$ values, which may indicate that $\left[{ }^{89} \mathrm{Zr}\right]$ beva has tumor specific binding, possibly to tumor VEGF. These results indicate that $\left[{ }^{89} \mathrm{Zr}\right]$ bevacizumab is a promising tracer for further development in clinical studies, aiming to identify patients who may benefit from bevacizumab therapy.

\section{7}

Investigating molecular imaging biomarkers as preliminary indicators of treatment response in a triple-negative model of breast cancer. S.L. Barnes, J.G. Whisenant, T.E. Yankeelov; Vanderbilt University, Nashville, TN

Introduction: The current standard method for assessing treatment response in cancer (RECIST) relies on monitoring changes in the physical size of the tumor. However, therapeutic approaches generally target specific molecular pathways, initializing events that occur at the cellular level in response to treatment prior to the manifestation of a change in the size of the tumor. For this reason, evaluation of the tumor size may not be the most sensitive metric for treatment response. Additionally, with the development and advancement of molecular imaging techniques that allow for monitoring of the tumor microenvironment, the potential for imaging biomarkers as early indicators of treatment response is eminent. The purpose of this work is to investigate the potential of the apparent diffusion coefficient (ADC) from DW-MRI, $K^{\text {trans }}$ from DCE-MRI, and the standardized uptake value (SUV) from ${ }^{18} \mathrm{~F}$-FDG PET as indicators of treatment response in a preclinical model of triple negative breast cancer (TNBC). Methods: Four to five-week old female nude mice were injected subcutaneously in the hind limb with approximately $1 \times 10^{6}$ MDA-MB-231 cells. Tumors were allowed to grow until they reached a volume of approximately $200-300 \mathrm{~mm}^{3}$, at which point the mice were entered into the study. The study consisted of three imaging sessions (baseline, day 2, and day 4) and two treatments (after imaging on baseline and day 3 ). The mice were divided into three groups: control which received i.p. saline injections, a "low" dose treatment group which received $15 \mathrm{mg} / \mathrm{kg}$ of Abraxane (a chemotherapy used clinically for treatment of TNBC), and a "high" dose treatment group which received a $25 \mathrm{mg} / \mathrm{kg}$ of Abraxane. At the time of submission, five mice were scanned for the control group, four for the low dose treatment group, and five for the high dose treatment group. MRI was performed on a Varian 7.0T scanner and included DW-MRI, DCE-MRI, and anatomical scans. PET imaging was performed on a microPET Focus 220 system and utilized ${ }^{18} \mathrm{~F}-\mathrm{FDG}$. Animals remained anesthetized throughout the entire imaging session and were maintained in position during transfer between imaging modalities by means of a specially-designed animal holder. The DW-MRI protocol employed a gated and navigated pulsed gradient spin echo sequence with b-values of 150,500 , and $800 \mathrm{~mm}^{2} / \mathrm{s}$; the ADC value was calculated for each voxel using standard methods. The DCE-MRI protocol employed a $T_{1}$-weighted, gradient echo sequence. A bolus injection of $120 \mu \mathrm{L}$ of 0.05 $\mathrm{mmol} / \mathrm{kg}$ Gd-DTPA was given after approximately 3 minutes of baseline collection, and data was collected for 20 minutes after injection. The data was then fit on a voxel-wise basis to the standard Kety-Tofts equation to extract $K^{\text {trans }}$. The standard uptake value (SUV) was calculated from the PET data for each voxel. For each of the three parameters (ADC, $K^{\text {trans }}$, SUV), the median value over the entire tumor volume was calculated for each imaging time point. The median values for each time point were then normalized by the baseline value, and the average value was calculated for each of the three treatment groups. Results: While no parameter was found to be significantly different between groups, this is likely due to the small group size, and additional imaging studies are currently underway to increase the number of animals in each group. However, trends can still be observed in the current data set. For example, the $25 \mathrm{mg} / \mathrm{kg}$ treatment group shows a larger change (23\%) between baseline and day 2 of the SUV than the other groups; since at day 2 there is no significant difference in tumor volume ( $p>0.05$ in all cases) between any groups, the SUV may prove to be an early biomarker of treatment response. With regard to the $15 \mathrm{mg} / \mathrm{kg}$ group, $\mathrm{ADC}$ and $K^{\text {trans }}$ seem particularly promising for differentiation from the control group. Importantly, the MDA-MB-231 tumors demonstrate both necrosis and apparent regions of fluid retention in histology which will potentially affect the median tumor parameter values. Hence, future analysis will concentrate on identifying and removing necrotic voxels. Conclusions: This work represents preliminary results of a treatment response study. Though the data is currently limited, thus making achieving significance difficult, patterns of imaging parameter trends can be seen in the data that are promising in regards to determining imaging parameters that can serve as early indicators of treatment response.

\section{8}

Imaging of intratumoral inflammation during oncolytic virotherapy of tumors by ${ }^{19} \mathrm{~F}$ magnetic resonance imaging (MRI). S. Weibel ${ }^{1}$, T. BasseLuesebrink $^{1}$, M. Hess ${ }^{1}$, E. Hofmann ${ }^{1}$, C. Seubert ${ }^{1}$, J. Langbein-Laugwitz ${ }^{1}$, I. Gentschev ${ }^{1}$, J. Sturm ${ }^{2}$, Y. Ye ${ }^{1}$, T. Kampf ${ }^{1}$, P. Jakob ${ }^{1}$, A.A. Szalay ${ }^{1}$; ${ }^{1}$ University of Wuerzburg, Wuerzburg, Germany, ${ }^{2}$ Medical University Hospital, Tuebingen, Germany

Oncolytic virotherapy of tumors is an up-coming, promising therapeutic modality of cancer therapy. Unfortunately, non-invasive techniques to evaluate the inflammatory host response to treatment are rare. Here, we evaluate ${ }^{19} \mathrm{~F}$ magnetic resonance imaging (MRI) which enables the noninvasive visualization of inflammatory processes in pathological conditions by the use of perfluorocarbon nanoemulsions (PFC) for monitoring of oncolytic virotherapy. The vaccinia virus strain GLV-1h68 was used as an oncolytic agent for the treatment of different tumor models. Systemic application of PFC emulsions followed by ${ }^{1} \mathrm{H} /{ }^{19} \mathrm{~F}$ MRI of mock-infected and GLV-1h68-infected tumor-bearing mice revealed a significant accumulation of the ${ }^{19} \mathrm{~F}$ signal in the tumor rim of virus-treated mice. Histological examination of tumors confirmed a similar spatial distribution of the ${ }^{19} \mathrm{~F}$ signal hot spots and CD68+-macrophages. Thereby, the CD68+-macrophages encapsulate the GFP-positive viral infection foci. In multiple tumor models, we specifically visualized early inflammatory cell recruitment in vaccinia virus colonized tumors. Furthermore, we documented that the ${ }^{19} \mathrm{~F}$ signal correlated with the extent of viral spreading within tumors. These results suggest ${ }^{19} \mathrm{~F}$ MRI as a non-invasive methodology to document the tumorassociated host immune response as well as the extent of intratumoral viral replication. Thus, ${ }^{19} \mathrm{~F}$ MRI represents a new platform to non-invasively investigate the role of the host immune response for therapeutic outcome of oncolytic virotherapy and individual patient response.

\section{9}

Molecular imaging of the premetastatic niche in the lungs of a metastatic mouse model of breast cancer. W. Beaino ${ }^{1}$, J.K. Schwarz ${ }^{2}$, M. Zahner $^{2}$, A. Zheleznyak ${ }^{2}$, M. Shokeen ${ }^{2}$, M. Jiang ${ }^{1}$, C.J. Anderson ${ }^{1}$; ${ }^{1}$ University of Pittsburgh, Pittsburgh, PA, ${ }^{2}$ Washington University, St. Louis, MO

The "pre-metastatic niche" occurs prior to the metastatic cascade, where niche is defined as the microenvironment for tumor cells to be able to engraft and proliferate at secondary sites. Bone marrow derived cells (BMDCs) expressing very late antigen-4 (VLA-4) have been shown to arrive at sites of metastasis to form a receptive environment for tumor cells. Here we describe an imaging strategy for BMDCs in the lungs of mice after i.v. injection of VLA-4 negative 4T1/luc mouse mammary carcinoma cells. Mice were imaged after 1-19 days post-tumor cell injection using bioluminescence imaging and PET imaging with a high affinity VLA-4 
tracer, ${ }^{64} \mathrm{Cu}-\mathrm{CB}-\mathrm{TE} 1 \mathrm{~A} 1 \mathrm{P}-\mathrm{LLP} 2 \mathrm{~A}$. PET imaging showed increasing uptake of ${ }^{64} \mathrm{Cu}-\mathrm{CB}-\mathrm{TE} 1 \mathrm{~A} 1 \mathrm{P}-\mathrm{LLP} 2 \mathrm{~A}$ with increasing tumor burden, and these data correlated to increasing HPC colonies measured in the blood. Studies to compare the i.v. injected tumor cells to metastasis from mammary fat pad tumors as well as fluorescence molecular tomography imaging studies with cypate-LLP2A are ongoing.

\section{0}

Newly detected lesions by early FDG-PET are significantly more predictive of OS than new lesions by DxCT in NSCLC patients treated with a Met-inhibitor. T. Bengtsson, S. Sanabria, A. Peterson, A. de Crespigny; Genentech, South San Francisco, CA

Purpose: We evaluate if newly detected lesions (NL) by early FDG-PET predict overall survival (OS) in the Phase II study of erlotinib (E) +/MetMAb (M) for advanced NSCLC (trial OAM4558g), and benchmark these results against those obtained by considering NL by diagnostic CT (NL-CT) determined at first radiological assessment. Experimental Procedures: FDG-PET scans were acquired at baseline and between days 10 and 14 in Cycle 1. Median time interval between the 2 scans was 3 weeks. PET scans were assessed by a single reviewer. A NL by FDG-PET was defined as an area of focal avidity at day 10-14 that was not apparent at baseline. Diagnostic CT scans were separately acquired at baseline and at day 42, with radiological assessments performed at the investigator site. Results: Compared to NL-CT, a significantly stronger and more robust correlation was found between NL-PET and OS. Specifically, adjusted for EGFR status, evaluated on a comparable subset of patients with OS greater than 42 days $(\mathrm{n}=96)$, median OS was 3.0 vs. 11.7 months for patients with and without NL-PET $(\mathrm{HR}=0.32, \mathrm{p}<7 \mathrm{e}-04)$. By comparison, based on NL-CT, the corresponding median OS values were 5.9 vs. 9.2 months $(\mathrm{HR}=0.53, \mathrm{p}<.056)$. Further, by multivariate analysis using the Cox model, day $42 \mathrm{NL}-\mathrm{CT}$ was not significant when adjusting for Met status, treatment assignment, EGFR status, and baseline ECOG ( $\mathrm{p}=.14)$. In contrast, by the same analysis, NL-PET remained highly significant $(\mathrm{p}<.0008)$. In known EGFR wildtype patients, no correlation was observed between changes in SUVmax and OS, including for Met Dx+ and Met Dx-. The NL-rate by FDG-PET was $19.0 \%$ vs. $21.6 \%$ for NL-CT. There was an approximate $50 \%$ agreement for patients with NL-PET and NL-CT, and a corresponding $85 \%$ agreement for patients without NL (Kappa $=.45$; 'fair' overall agreement). In Met Dx+ patients, the NL-rate by FDG-PET was approximately $10 \%$ for $\mathrm{M}$-treated patients compared with 30\% for the placebo group $(\mathrm{p}<.036)$. By comparison, based on diagnostic CT, the corresponding NL-rates were $22 \%$ for M-treated vs. $32 \%$ for placebo $(p=.21)$. An in-depth re-analysis of all patients with NL-PET suggested that patients could be classified into 4 different categories from "most" to "least informative" vis-à-vis OS. Set 3 consisted of those cases with obvious high uptake NLs at follow-up, with no evidence of FDG avidity at screening. Set 2 included cases with obvious high uptake NL but large tumor burden or low uptake NL, with no evidence of FDG avidity at screening. Set 1 consisted of cases with nearly new or equivocal NL, usually faintly visible at screening. And set 0 comprises cases in which NL was reported but re-analysis of the images revealed lesions which were clearly visible at screening. Based on this categorization, the HRs for sets 3 to 1 were $0.15(\mathrm{p}<3 \mathrm{e}-06), 0.32(\mathrm{p}<.035)$, and $0.45(\mathrm{p}=.21)$, respectively. Conclusions: The results suggest that a decrease in the rate of NL by FDG-PET may have potential as an early surrogate of clinical activity in randomized studies of therapies where radiological response may not be readily apparent or a-priori hypothesized. Specifically, in this study, NL by FDG-PET was found to be a strong and independent predictor of OS. In contrast, independent of clinical and baseline information, NL by diagnostic CT did not predict OS. A lower NL rate by FDG-PET was observed in the Met $\mathrm{Dx}+$ patients treated with MetMAb. Bright, newly detected lesions with no correlate at baseline, representing small, focal areas of rapidly growing tumor, are most predictive of short OS.

\section{$11^{*}$}

Molecular-genetic imaging of prostate cancer. A. Bhatnagar ${ }^{1}, \mathrm{~K}$. Gabrielson ${ }^{1}$, P.B. Fisher ${ }^{2}$, M.G. Pomper ${ }^{1}$; ${ }^{1}$ Johns Hopkins Medical
Institutions, Baltimore, MD, ${ }^{2}$ Virginia Commonwealth University School of Medicine, Richmond, VA

Objectives: We propose a radical new method for detecting prostate cancer (PCa), whether it is localized, or widespread and metastatic to bone. The method relies on the discovery of cancer-specific gene promoters, namely, progression elevated gene-3 (PEG-Prom) and astrocyte elevated gene-1 (AEG-Prom), which are up-regulated only in tumor and metastases. Our group has previously demonstrated the utility of the PEG-Prom to drive the expression of imaging reporters for two imaging modalities: firefly luciferase (Luc) for bioluminescence imaging (BLI) and herpes simplex virus type 1 thymidine kinase (HSV1-tk) for radionuclide imaging. This has been applied to experimental murine models of metastatic melanoma and breast cancer. We have recently shown that we could express the AEG-Prom selectively in PCa cells in vivo ( $\mathrm{n}=2)$, with the possibility of it being more robust than PEG-Prom. Here we propose that the systemic delivery of PEGProm- and AEG-Prom-driven constructs will enable a practical, new in vivo imaging technique that we will test in a model of $\mathrm{PCa}$ that is metastatic to bone. Methods: PEG-Prom- and AEG-Prom-driven imaging reporters for BLI and radionuclide imaging have been developed. The generated constructs have been tested in vitro for the function of reporter proteins. The enzymatic function of the Luc gene was validated by the luciferase assay and of the HSV1-tk component by an $\left[{ }^{125}\right.$ I]FIAU cell uptake assay. For testing the activities of the constructs in vivo, we used a linear polyethyleneimine (1-PEI) nanoparticle for intravenous (IV) delivery of the promoter constructs to animals bearing tumors derived from PC3-ML (human metastatic) cells along with controls (Ctrl). PEG-Prom- and AEGProm-driven imaging reporter gene expression was monitored by small animal BLI and by single photon emission computed tomography (SPECT)/ CT after IV administration of $\left[{ }^{125} \mathrm{I}\right]$ FIAU. After image acquisition and reconstruction, SPECT images were coregistered to the $\mathrm{CT}$ and quantified. Imaging (BLI) results were validated by analyzing the harvested tumor tissues and correlating them with histology, western blot and quantitative PCR. Results: Both BLI and SPECT/CT imaging demonstrated tumorspecific PEG-Prom and AEG-Prom activity in the PCa model, but not in the Ctrl. Metastatic nodules detected by the SPECT/CT imaging were confirmed by gross pathology. Using abdominal area as a region of interest (ROI), quantification of the BLI data $(\mathrm{n}=6)$ at the $48 \mathrm{~h}$ time point after reporter gene delivery showed 19-fold and 16-fold higher accumulation of Luc in the PCa group compared to the Ctrl for the AEG-Prom and PEG-Prom, respectively. BLI results were further confirmed by immunohistochemistry and western blot. Quantification of the SPECT data $(\mathrm{n}=2)$ at the $56 \mathrm{~h}$ time point after reporter gene or $8 \mathrm{~h}$ after [ $\left.{ }^{125} \mathrm{I}\right]$ FIAU delivery showed 11 -fold and 2.5 -fold higher accumulation of [ $\left.{ }^{125} \mathrm{I}\right] \mathrm{FIAU}$ in the PCa group compared to the Ctrl for AEG-Prom and PEG-Prom, respectively. Conclusions: The results demonstrate the potential of systemically delivered, PEG-Prom- and AEG-Prom-driven constructs for enabling the imaging of metastatic $\mathrm{PCa}$ in vivo.

\section{2}

Imaging mRNA decay dynamics in colorectal cancer. F.F. Blanco; The University of Kansas Medical Center, Kansas City, KS

Messenger RNA (mRNA) decay is a tightly regulated mechanism that serves as a fundamental means to control gene expression. In the intestinal epithelium, normal cell growth is controlled by rapid decay of growthrelated mRNAs. This class of mRNAs is targeted for degradation through AU-rich element (ARE) RNA motifs present in the mRNA 3' untranslated region (3'UTR). A unique aspect of this post-transcriptional regulation is that it can be visualized through the presence of cytoplasmic processing (P-) bodies that contain various components of mRNA degradation machinery. Here, we demonstrate in non-transformed intestinal epithelial cells that the mRNA decay factor tristetraprolin (TTP) binds and delivers ARE-containing mRNAs to P-bodies where the mRNA is degraded. Co-localization studies and $3 \mathrm{D}$ reconstruction analysis revealed an association between TTP and various P-body components such as the decapping protein Dcp1a upon TTP-driven delivery of ARE-mRNAs to P-bodies. However, P-body formation was impaired in colorectal cancer cells and oncogenic Rastransformed intestinal epithelial cells. More importantly, P-body levels were significantly reduced in colorectal adenomas and adenocarcinomas compared to normal colonic epithelium. Consistent with loss of P-body 
formation, transformed cells displayed concurrent loss of TTP expression, and expression profiling revealed TTP to be lost in $>75 \%$ of colorectal adenocarcinomas. The functional consequence of TTP loss is observed with a 3- to 4-fold enrichment in ARE-containing gene expression in colorectal adenocarcinomas. In agreement with these observations, colonic epithelium from TTP-deficient mice show diminished P-body numbers and elevated pro-inflammatory gene expression. Furthermore, restoring TTP expression in cancer cells resulted in rescue of P-body assembly accompanied by TTPdependent growth-inhibition. These findings implicate TTP as a central physiological driver of ARE-mediated mRNA decay by virtue of its ability to sequester ARE-mRNAs and nucleate cytoplasmic P-bodies, and loss of this mechanism contributes to colorectal cancer progression.

\section{3}

Multilayered valency: a novel design of peptidic imaging agents for the integrin $\alpha \mathbf{v} \beta 6$. K.C. Brown, A.N. Singh, S. Li, G. Hao, A. Kumar, M.J. McGuire, X. Sun; The University of Texas Southwestern Medical Center, Dallas, TX

Non-small cell lung cancer is the largest cancer killer in the United States. The lethality of NSCLC is due in part to late diagnosis, tumor heterogeneity, and rudimentary tumor classification. The integrin $\alpha v \beta 6$ is over-expressed in NSCLC as well as other epithelial-derived cancers. By contrast, its expression is low to undetectable in normal tissue. Expression of $\alpha v \beta 6$ occurs early in NSCLC progression and is correlated with poor prognosis. We evaluated an $\alpha v \beta 6$-binding peptide selected from a phage-displayed library for PET imaging of $\alpha \mathrm{v} \beta 6+$ NSCLC. Multimeric presentation of the peptide RGDLATLRQL on a bifunctional copper chelator was achieved using two approaches: dimerization of the peptide followed by conjugation to the chelator $\left(\mathrm{H}_{2}-\mathrm{D} 10\right)$ and direct presentation of two copies of the peptide on the chelator scaffold $\left(\mathrm{H}_{2}-(\mathrm{M} 10)_{2}\right)$. Binding affinities of the divalent peptide conjugates are four-fold higher than their monovalent counterpart $\left(\mathrm{H}_{2}-\mathrm{M} 10\right)$, suggestive of multivalent binding. PET imaging using the bivalent ${ }^{64} \mathrm{Cu}$-labeled conjugates showed rapid and persistent accumulation in $\alpha v \beta 6+$ tumors. By contrast, no significant accumulation was observed in $\alpha v \beta 6$ - tumors. Irrespective of the dimerization approach, all divalent probes showed three-fold higher tumor uptake than the monovalent probe, indicating the role of valency in signal enhancement. However, the divalent probes have elevated uptake in non-target organs, especially the kidneys. To abrogate nonspecific uptake, the peptide's N-terminus was acetylated. The resultant bivalent probe, ${ }^{64} \mathrm{Cu}-\mathrm{AcD} 10$, showed drastic decrease of kidney accumulation while maintaining tumor uptake. In sum, we developed an $\alpha \mathrm{v} \beta 6$-integrin specific probe with optimized biodistribution for noninvasive PET imaging of NSCLC. Further, we have demonstrated that use of multivalent scaffolds is a plausible method to improve library selected peptides, which would be suboptimal or useless otherwise, for imaging probe development.

\section{4}

Inhomogeneity in FDG-uptake for therapy response assessment for neoadjuvant therapy in colorectal cancer: comparison with histopathology. R.A. Bundschuh ${ }^{1}$, L. Neumann ${ }^{2}$, M. Seyfried ${ }^{2}$, J. Dinges ${ }^{2}$, K. Herrmann ${ }^{1}$, M. Essler ${ }^{2}$; ${ }^{1}$ Dept. of Nucl. Medicine, Universitätsklinikum Würzburg, Wuerzburg, Germany, ${ }^{2}$ Dept. of Nucl. Medicine, Klinikum Rechts der Isar der TU München, Munich, Germany

Introduction: Fluordesoxyglucose positron emission tomography (FDGPET) has been shown to predict early therapy response to neoadjuvant chemo- and/or radiotherapy in several tumor entities. Normally changes in glucose uptake (SUV) are used as a measure. Recently the inhomogeneity of glucose uptake was found to be a promising new prognostic factor (1). Therefore we investigated the use of inhomogeneity for therapy monitoring in neoadjuvant chemo-radiotherapy (RCTx) for colorectal cancer with histopathology as gold standard. Methods: 27 patients with proven colorectal carcinoma were included in this study. All patients underwent FDG-PET/CT before therapy, after 14 days of initiation of neoadjuvant RCTx and when the therapy was finished. Consequently primary tumor was resected and histopathologically worked up. In all PET/CT data, the tumor was delineated manually in CT data and consequently maximum diameter, tumor volume, maximum SUV, mean SUV, and total lesion glycolysis were estimated as standard parameters. In addition coefficient of variance (COV), skewness and kurtosis of the lesion were calculated as parameters for inhomogeneity. Differences between the early and the late PET examination compared to the pretherapeutic PET were calculated. ROC analysis was performed to estimate the accuracy for all parameters to identify histopathological response. Results: 19 patients were identified as therapy responders by histopathological criteria. For the early time point COV showed the highest area under the curve of 0.84 , which was statistically significantly $(\mathrm{p}<0.05)$ higher than the best standard parameter, which was the maximum lesion diameter with a value of 0.71 . Sensitivity for the prediction of histological response was $77 \%$, specificity was $75 \%$, positive predictive value (PPV) was $88 \%$ and negative predictive value (NPV) was $60 \%$. COV showed also the highest area under the curve when the PET data acquired after therapy was finished were used. Sensitivity of COV to predict histopathological therapy response was $85 \%$, and specificity was $75 \%$, resulting in a PPV of $90 \%$ and a NPV of $86 \%$. We found that even the COV in the baseline scan had an area under the curve of 0.76 and therefore a higher prognostic value for the therapy response assessment as the best conventional parameter; however, this difference was not significant. Conclusions: $\mathrm{COV}$ was found to be the best parameter to assess therapy response in patients with colorectal cancer treated with neoadjuvant RCTx. Therefore future work should be focused on the application of COV for early therapy response assessment of FDG-PET data instead of conventional parameters such as SUV. Future studies need to be done to assess the use of $\mathrm{COV}$ for prediction of patient outcome and also for other tumor entities. Whether COV measured in the baseline scan only yields prognostic information for therapy response of neoadjuvant RCTx needs to be investigated in the future as well. Reference: 1 . Tixier F, Le Rest CC, Hatt M, Albarghach N, Pradier O, Metges JP, Corcos L, Visvikis D. Intratumor heterogeneity characterized by textural features on baseline 18F-FDG PET images predicts response to concomitant radiochemotherapy in esophageal cancer. J Nucl Med. 52(3):369-78, 2011.

\section{5}

Neutron radiography combined with neutron-computed tomography: a novel tool for cancer diagnosis and imaging. M. Cekanova ${ }^{1}$, H.Z. Bilheux $^{2}$, K. Rathore ${ }^{1}$, J.C. Bilheux ${ }^{2}$, L. Walker ${ }^{2}$, R. Donnell', A.M. Legendre ${ }^{1} ;{ }^{1}$ The University of Tennessee, College of Veterinary Medicine, Knoxville, TN, ${ }^{2}$ Oak Ridge National Laboratory, Oak Ridge, TN

The early detection and correct staging of cancer are important for successful treatment of patients with cancer. Development of imaging modalities to provide information about structure and cellular/molecular composition of tumor is required. Neutron radiography (NR) combined with neutron computed tomography $(\mathrm{nCT})$ are novel imaging tools to image and provide additional characterization of the tumor. Neutron imaging is based on the attenuation, both scattering and absorption, of a directional neutron beam by the matter through which it passes. Hydrogen nuclei scatter thermal and cold neutrons more strongly than other atomic nuclei; thus hydrogen is a primary contributor to neutron contrast of biological specimens. Because of increased levels of water and changes in protein and lipid production in tumor cells, neutron imaged tissues provide important information about molecular changes in the tumor. We utilized NR combined with $\mathrm{nCT}$ to evaluate the transmission of neutrons in normal tissue and compared it with cancer tissues from dogs with naturally occurring tumors. The normal and adenocarcinomas tissues of breast, kidney, liver, and lung were cut into $2 \times 2 \times 1 \mathrm{~cm}$ pieces, fixed in $10 \%$ BNF or $75 \%$ deuterated ethanol overnight. The fixed tissues were imaged by $\mathrm{NR}$ and $\mathrm{nCT}$ at the CG-1D neutron imaging prototype beam-line at the High Flux Isotope Reactor facility of the ORNL. The CG-1D beam-line was equipped with an aperture that defines the beam geometry, a $5 \mathrm{~m}$ long He-filled flight tube for optimum neutron transport, and a combined translation/rotation sample stage for $2 \mathrm{D}$ and $3 \mathrm{D}$ imaging. Neutron radiographs of each tissue specimen were taken for $3 \mathrm{~min}$. The quantification and data normalization of the neutron transmission was performed using custom-made code based on MATLAB ${ }^{\circledR}$. The normalization procedure included removal of background effects and beam fluctuations. Mean transmission intensity values of the tissues were calculated by summing the intensity of each pixel and dividing by the number of pixels occupied by the tissue. Octopus software was used to reconstruct the data and thus provide $\mathrm{CT}$ slices. Our preliminary data show that deuterated ethanol fixation is a better fixative than formalin for the 
neutron imaging of biological specimens. As expected, the stroma containing adipose and connective tissues composed of the lipids and collagen had higher neutron transmission than areas containing hydrogen rich adenocarcinomas. Adenocarcinomas had lower neutron transmission on neutron radiographs. Neutron radiographs were similar to optical microscopy images with detailed histology from hematoxylin and eosin stained tissue. Neutron radiography and $\mathrm{nCT}$ are novel technologies to obtain complementary information about tumors at very high spatial resolution $(50 \mu \mathrm{m})$ with the ability to differentiate tumors from surrounding normal tissues. Future development of neutron imaging may be able to identify certain elements in the tissues, which may better identify tumor type, tumor margins, and assess the response to chemo- and radiotherapy evaluating the pre- and post-treatment biopsy specimens. NR and $\mathrm{nCT}$ is a novel imaging modality that may complement currently used MRI, and CT to obtain additional information about tumor structure and its cellular/ molecular composition

\section{6}

The additional value of nonenhanced CT acquired during ${ }^{18}$ F-FDG PET/CT in prediction of I-131 ablation success. A. Cho, J. Lee, M. Yun; Yonsei University Health System, Seoul, Korea (Rep.)

Purpose: The high content of iodine in thyroid parenchyma is the cause for high Hounsfield Unit (HU) in pre-contrast CT. A potential important factor in predicting I-131 ablation success is the innate iodine concentration ability of thyroid parenchyma. We theorize that the iodine content in the normal thyroid parenchyma measured by HU acquired during PET/CT can predict I-131 ablation success after surgery. Materials and Methods: A total of 131 patients who underwent PET/CT before surgery from January 2005 to December 2009 were selected for this study. These patients underwent low dose I-131 ablation $(30 \mathrm{mCi}, 94$ patients) or high dose I-131 ablation $(150 / 200 \mathrm{mCi}, 24$ patients) within one year of surgery, and then had a second diagnostic I-131 whole body scans $(5 \mathrm{mCi})$ after one year of initial I-131 ablation. Stimulated serum TSH, thyroglobulin (Tg), thyroglobulin antibody $(\mathrm{TgAb})$ were taken before I-131 ablation and follow up unstimulated TSH, $\mathrm{Tg}, \mathrm{TgAb}$ levels were intermittently taken before and after I-131 whole body scans. Thyroid iodine content was measured by drawing ROI on the thyroid parenchyma and mean HU was recorded. 1st and 2nd I-131 whole body scan (WBS) images were evaluated for I-131 uptake in the neck. Increased I-131 uptake in the neck in the 2nd diagnostic I-131 WBS was considered to be imaging ablation failure. Serum $\mathrm{Tg}, \mathrm{TgAb}$ levels were also used to evaluate for ablation success. Stimulated Tg levels higher than 5ng/ $\mathrm{ml}$ or unstimulated $\mathrm{Tg}$ levels higher than $1 \mathrm{ng} / \mathrm{ml}$ were considered to be serum ablation failure. Results: 70 out of 131 patients had no I-131 uptake in the neck in the 2nd I-131 WBS. Pre-contrast CT HU was significantly higher in 2nd I-131 ablation success patients than in patients that had residual I-131 uptake in the 2 nd I-131 WBS $(108.1 \pm 17$ vs $98.5 \pm 19.17$, $\mathrm{p}=0.005$ ). ROC analysis showed that a cut-off of 103.5 had the highest sensitivity in predicting $\mathrm{I}-131$ ablation success (AUC $=0.683, \mathrm{p}<0.001$ ). Using this cut-off value, the sensitivity, specificity and accuracy was $71 \%$, $63 \%$ and $67 \%$. High dosage of I-131 was also predictive of 2nd I-131 WBS success $(\mathrm{x} 2, \mathrm{p}=0.027)$. There was no correlation between pre-contrast CT HU with serum ablation failure criteria. Conclusion: Pre-contrast CT acquired during PET/CT before surgery is helpful in predicting I-131 ablation success in thyroid cancer patients. Iodine content in the thyroid parenchyma may be an important factor in predicting I-131 ablation success.

\section{7}

Bioluminescence imaging for assessment of drug efficacy in human leukemia cell lines: effects of monoclonal and polyclonal cell populations on intensity and kinetics of photon emission. S. Christoph ${ }^{1}$, J. Schlege ${ }^{1}$, F. Alvarez-Calderon ${ }^{1}$, Y.M. Kim ${ }^{2}$, L.N. Brandao ${ }^{1}$, D. DeRyckere ${ }^{1}$, D.K. Graham ${ }^{1}$; ${ }^{1}$ University of Colorado Denver, Aurora, CO, ${ }^{2}$ Children's Hospital Los Angeles, Los Angeles, CA

Introduction: Although animal models of leukemia often utilize survival time as the primary therapeutic end point, bioluminescence imaging (BLI) is increasingly being used to provide quantitative and more rapid assessment of drug efficacy in pre-clinical oncology research. In this study we investigated the utility of BLI using a firefly luciferase system with monoclonal and polyclonal human leukemia cell populations (697, Jurkat, K562) in vitro and in a xenograft mouse model. Methods: Monoclonal and polyclonal human lymphoid and myeloid leukemia cell lines transduced with firefly luciferase were used for BLI. Results: Kinetics and dynamics of bioluminescence signal were cell line dependent. Instability and incomparability of luciferase activity were observed in polyclonal human leukemia cell lines in vitro. Luciferase expression decreased significantly over time in polyclonal leukemia cells. Inconsistent luciferase activity in a xenograft mouse model after transplantation of polyclonal luciferasetagged leukemia cell lines was detected. The bioluminescence intensity varied from mouse to mouse between $1.8 \times 10^{6}$ photons/second and $13 \times 10^{6}$ photons/second, equivalent to a greater than 7 -fold difference in bioluminescence intensity. After selection of monoclonal cell populations, luciferase activity was stable, equal kinetic and dynamic of bioluminescence intensity and strong correlation between cell number and light emission in vitro were observed. We obtained an equal development of leukemia burden detected by luciferase activity in NOD scid gamma mice after transplantation of monoclonal populations. Conclusion: The use of monoclonal leukemia cells selected for stable and equal luciferase activity is recommended for experiments in vitro and xenograft mouse models, especially for the assessment of drug efficacy in pre-clinical oncology research.

\section{8}

MicroPET imaging of CD13 receptor expression using a novel ${ }^{64} \mathrm{Cu}$-labeled NGR peptide constructed via bioorthogonal click chemistry. G. $\mathrm{Li}^{1}$, X. Wang ${ }^{1}, \mathrm{~S}$. Zong ${ }^{2}$, J. Wang ${ }^{2}$, K. Chen ${ }^{1}$, P.S. Conti ${ }^{1}$; ${ }^{1}$ University of Southern California, Los Angeles, CA, ${ }^{2}$ The Fourth Military Medical University, Xi'an, Shaanxi, China

Objectives: The NGR-containing peptides have been shown to specifically bind to CD13 receptor, one of attractive tumor vasculature biomarkers. In this study, we synthesized a novel ${ }^{64} \mathrm{Cu}$-labeled NGR peptide via bioorthogonal click chemistry and evaluated the newly developed probe for microPET imaging of CD13 receptor expression in vivo. Methods: Western blot analysis and immunofluorescence staining were performed to identify the CD13-positive and CD13-negative cell lines. Macrocyclic chelating agent 1,8-diamino-3,6,10,13,16,19-hexaazabicyclo[6.6.6]eicosine (DiAmSar) was conjugated with an azide moiety and radiolabeled with positron emitter ${ }^{64} \mathrm{Cu}\left(\mathrm{t}_{1 / 2}=12.7 \mathrm{~h}\right)$ in ammonium acetate buffer. The resulting ${ }^{64} \mathrm{Cu}$-labeled synthon was mixed with an alkyne-terminated NGR peptide to afford the final ${ }^{64} \mathrm{Cu}$-labeled NGR peptide $\left({ }^{64} \mathrm{Cu}-\mathrm{Sar}\right.$ NGR). Subsequently, ${ }^{64} \mathrm{Cu}$-Sar-NGR was evaluated in in vitro stability, tumor cell uptake and retention, small animal PET, and biodistribution studies. Results: The conjugation between ${ }^{64} \mathrm{Cu}$-labeled azide-containing DiAmSar and alkyne-terminated NGR peptide was completed in a quantitative yield within $15 \mathrm{~min}$ of mixing at low micromolar concentrations. ${ }^{64} \mathrm{Cu}$-Sar-NGR showed very good stability in mouse serum at $37^{\circ} \mathrm{C}$ with more than $93 \%$ of parent probe unchanged after $24 \mathrm{~h}$ of incubation. In vitro studies demonstrated that CD13 receptors are overexpressed in human fibrosarcoma HT-1080 cells and negative in human breast adenocarcinoma MCF7 cells. The cell uptake study revealed that ${ }^{64} \mathrm{Cu}$-Sar-NGR binds to CD13-positive HT-1080 cells, but not to CD13-negative MCF7 cells. For microPET study, ${ }^{64} \mathrm{Cu}-\mathrm{Sar}-\mathrm{NGR}$ displayed favorable in vivo performance in terms of good tumor uptake and retention in nude mice with HT-1080 tumor xenografts. As anticipated, significantly lower tumor uptake was observed for ${ }^{64} \mathrm{Cu}-\mathrm{Sar}-\mathrm{NGR}$ in CD13-negative MCF7 tumor xenografts. The CD13-specific tumor activity accumulation of ${ }^{64} \mathrm{Cu}$-Sar-NGR was further demonstrated by significant reduction of tumor uptake in HT-1080 tumor xenografts with a co-injected blocking dose of non-radiolabeled NGR peptide. The biodistribution results were consistent with the quantitative analysis of microPET imaging, demonstrating good tumor/muscle ratio $(7.48 \pm 0.21)$ of ${ }^{64} \mathrm{Cu}$-Sar-NGR at $18 \mathrm{~h}$ postinjection in HT-1080 tumor xenografts. Conclusions: The strain-promoted click chemistry approach has been successfully applied to the development of ${ }^{64} \mathrm{Cu}$-labeled NGR peptide. MicroPET imaging and biodistribution studies revealed significant and specific tumor uptake of the ${ }^{64} \mathrm{Cu}$-labeled "click" NGR probe in CD13positive HT-1080 tumor xenografts. This new approach is versatile, which warrants rapid and robust construction of highly diverse radiometalated bioconjugates for radioimaging and radiotherapy. 
Evaluation of human mannose receptor (CD206) binding of Tc 99m tilmanocept: a novel biotargeted sentinel node mapping agent for solid tumors. A.K. Azad ${ }^{1}$, M.V.S. Rajaram ${ }^{1}$, W.L. Metz ${ }^{2}$, F.O. Cope ${ }^{2}$, R.D. Hartman ${ }^{2}$, M.T. Joy ${ }^{2}$, B.M. Potter ${ }^{2}$, B.C. Abbruzzese ${ }^{2}$, M.S. Blue ${ }^{2}$, L.S. Schlesinger ${ }^{1},{ }^{1}$ The Ohio State University Wexner Medical Center and Comprehensive Cancer Center, Columbus, OH, ${ }^{2}$ Navidea Biopharmaceuticals, Inc., Dublin, $\mathrm{OH}$

Purpose: This study was designed to characterize the binding of Tc $99 \mathrm{~m}$ tilmanocept to its intended target, the mannose receptor (MR; CD206) expressed on human macrophages (MФs). Tilmanocept is a synthetic radiolabeled ligand designed to provide high sensitivity and specificity for lymphatic mapping (ILM) of tumor-draining lymph nodes (LNs). Tilmanocept is a synthetic small nanomolecule consisting of multiple units of DTPA and mannose, each synthetically attached to a $10-\mathrm{kDa}$ dextran backbone, designed to target the mannose binding domains of the MR of MФs. Tilmanocept's small diameter permits enhanced diffusion into lymph nodes and blood capillaries, resulting in a rapid injection site clearance. This study was designed to evaluate the binding of tilmanocept to its intended target, the mannose receptor (CD206) expressed on human macrophages. Methods: Human monocyte-derived macrophages (MФs) derived from peripheral blood mononuclear cells were pre-incubated without/with an excess amount of cold (non-labeled) tilmanocept, followed by incubation in the presence of increasing concentrations of Cy3-tilmanocept and analyzed by flow cytometry. Human Embryonic Kidney 293 (HEK293) cells were transfected with an MR-expressing plasmid, lysed, and proteins were separated by SDS-PAGE. The Western blot was blocked and pre-incubated with/without cold tilmanocept. The blot was incubated with Tc $99 \mathrm{~m}$ tilmanocept and exposed to X-ray film. Human MФs were pre-incubated with non-labeled tilmanocept, followed by $\mathrm{Cy} 3$-tilmanocept. Cells were fixed, stained with 4', 6-diamidino-2-phenylindole (DAPI), and microscopically analyzed. Binding of tilmanocept to the MR was confirmed by siRNA knockdown. Images of fluorescent binding were obtained and mean fluorescence intensity was quantified. LN tissue sections from clinical trials with tilmanocept were blocked incubated with primary antibody followed by Alexa Fluor-conjugated secondary antibody, stained with DAPI, and microscopically examined. Results/Conclusions: The MR is abundantly expressed on human MФs. Tilmanocept dose-dependently binds to human MФs, but not to lymphocytes, and competitively inhibits its own binding, suggesting specific binding to the MR. Tilmanocept performed as a ligand with specific binding to CD206. Significant reduction in binding to mannan

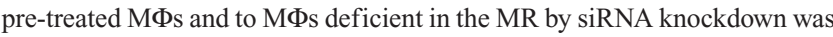
observed. The results strongly support that the MR is the binding receptor of tilmanocept. A clinically pathologically (path) negative (-) LN reveals the presence of resident MR-expressing MФs and does not appear to contain resident dendritic cells (DCs). The application of data from this model may be useful in utilizing tilmanocept for ILM and sentinel node biopsy in multiple solid tumor types.

\section{0}

Calibration of a Cerenkov luminescence imaging probe for prostate cancer detection. K. Deh ${ }^{1}$, S. Fareedy ${ }^{2}$, J. Osborne ${ }^{2} ;{ }^{1}$ Weill Cornell Medical College, New York, NY, ${ }^{2}$ Memorial Sloan-Kettering Cancer Center, New York, NY

Background: Prostate cancer (PCa) is the most common solid tumor in American males, and is estimated to kill one man in the U.S. every 18 minutes in 2012. Most men are diagnosed through biopsy after the detection of an abnormal prostate specific antigen (PSA) result, a procedure that has received the criticism of engendering prostate cancer over-treatment. Although this criticism is valid, it fails to address the root cause of the problem, which is that, even with the aid of ultrasound guidance, needle biopsy for prostate cancer diagnosis is a blind procedure. What might be useful is a real-time optical imaging system that illuminates cancerous lesions, guiding the clinician to obtain samples at these foci. Additionally, researchers have demonstrated that optical imaging has the potential for use in distinguishing between cancerous and benign lesions. Objectives: The objective of the study was to correlate strength of Cerenkov optical emissions from the prostate with the Gleason score to determine if cancer aggressiveness could be determined using this method. Methods: This project takes advantage of another project for imaging prostate cancer patients using ${ }^{89} \mathrm{Zr}$-DFO-J591, a radiolabeled monoclonal antibody that binds to the extracellular epitope of the prostate specific membrane antigen (PSMA), a membrane glycoprotein overexpressed in prostate cancer cells and upregulated in high grade disease. Charged particles emitted by ${ }^{89} \mathrm{Zr}$-DFO-J591, emit Cerenkov luminescence (CL) when their phase velocity exceeds the speed of light. Patients with localized prostate cancer scheduled to undergo radical prostatectomy were administered 15 to $20 \mathrm{mCi}$ of ${ }^{89} \mathrm{Zr}$-DFO-J591 3 to 5 days prior to surgery. After radical prostatectomy, the excised prostate gland was imaged ex-vivo in small animal imaging systems to allow a direct, close-up study of ${ }^{89} \mathrm{Zr}$-DFO-J591 without the attenuation resulting from surrounding soft and bony tissue inherent in in-vivo imaging. The excised prostate was imaged in animal MRI, animal PET and Cerenkov Luminescence Imaging System. Conclusions: The results established that the concentration of radioactivity sequestered in an average sized prostate cancer lesion in a man injected with $5 \mathrm{mCi}$ of ${ }^{89} \mathrm{Zr}$-DFO-J591 is on the order of $100 \mathrm{nCi} / \mathrm{cc}$. These experiments provided data for constructing phantoms and mouse models for calibration of prostate cancer optical endoscopic imaging device.

\section{1}

A novel diagnostic strategy for PET to define the clinical significance of the magnitude of target inhibition for therapies suppressing PI3K pathway signaling in renal cell carcinoma. M.G. Doran, S.M. Cheal, M.J. Evans, S.M. Larson, C.L. Sawyers, J.S. Lewis; Memorial Sloan-Kettering Cancer Center, New York, NY

Although inhibitors of PI3K pathway signaling (e.g. everolimus, temsirolimus) confer a survival benefit in some patients with metastatic renal cell carcinoma $(\mathrm{RCC})$, responses are variable and short-lived, and the overall merit of this therapeutic strategy for managing RCC is controversial. One manner in which the generally poor clinical responses could be rationalized is that the drug doses used in standard of care or in clinical trials do not inhibit PI3K pathway signaling to the extent required to confer a tumor response. In this regard, a noninvasive technology that quantitatively measures the activity of this oncogenic signaling pathway could more clearly define the clinical significance of the magnitude of target inhibition for PI3K pathway inhibitors in RCC. Appreciating that the loss of the tumor suppressor VHL sensitizes tumors to inhibitors of PI3K pathway signaling by suppressing downstream HIF $1 \alpha$ transcriptional activity, we reasoned that a HIF $1 \alpha$ target gene could serve as a noninvasive biomarker of PI3K pathway activity. To this end, we have developed ${ }^{89} \mathrm{Zr}$-labeled cG250, a radiotracer derived from the chimeric monoclonal antibody cG250, itself directed to Carbonic Anhydrase IX, a thoroughly validated HIF1 $\alpha$ target gene. ${ }^{89} \mathrm{Zr}-\mathrm{G} 250$ shows high affinity for CAIX in multiple preclinical models of VHL-null RCC in vitro and in vivo. Moreover, ${ }^{89} \mathrm{Zr}$-cG250 effectively measures treatment induced changes in CAIX expression with a panel of mechanistically unrelated targeted therapies inhibiting discrete nodes of the PI3K signaling pathway. Finally, ${ }^{89} \mathrm{Zr}$-cG250 PET effectively quantified the changes in CAIX expression post therapy that were indicative of PI3K pathway and HIF $1 \alpha$ inhibition (and eventual tumor response). Collectively, these results promote a novel and immediately translatable diagnostic strategy to monitor PI3K pathway signaling in RCC by measuring relative changes in CAIX expression with a radiolabeled antibody already cleared for clinical use.

\section{2}

Utility of quantitative-based $\left[{ }^{18} \mathrm{~F}\right] \mathrm{FDG}-\mathrm{PET}$ imaging for assessing therapy response and relapse in a transgenic mouse model of human multiple myeloma. K.M. Duncan, S.A. Walsh, J.J. Sunderland, L.L. Boles-Ponto, T.R. Rosean, S. Janz; The University of Iowa, Iowa City, IA

This study aims to quantify responses to proteasomal inhibition in a bitransgenic mouse model of human multiple myeloma designated as $\mathrm{C}$. IL6/iMyc utilizing $\left[{ }^{18} \mathrm{~F}\right]$ FDG-PET and to correlate these findings with serum immunoglobulin levels. C.IL6/iMyc mice $(n=6 ; 97-107$ days) were imaged to confirm the presence of tumors. Within two days of initial imaging, treatment was initiated (i.v.) with a $7 \mathrm{mg} / \mathrm{kg}$ dose of secondgeneration proteasome inhibitor MLN2238 (twice per week). To noninvasively monitor therapy response, two additional $\left[{ }^{18} \mathrm{~F}\right] \mathrm{FDG}-\mathrm{PET}$ images (post therapy 1; after 4-7 treatments and post therapy 2; terminal images) 
were acquired after treatment was initiated. As an additional biomarker of drug response, weekly serum samples were extracted and serum immunoglobulin levels were quantified. Baseline (pre-therapy) $\left[{ }^{18} \mathrm{~F}\right] \mathrm{FDG}$ PET images confirmed the presence of metabolically active tumors in the spleen and cervical, axillary, inguinal and mesenteric lymph nodes (CLN, ALN, ILN and MLN, respectively). Four to seven doses of MLN2238 were effective in significantly reducing the mean SUVmax values in the MLN from $2.8 \pm 0.6$ to $1.9 \pm 0.3(\mathrm{p}<0.05)$. Similar reduction in metabolic activity was observed in the spleen and peripheral lymph nodes. Furthermore, seven doses of MLN2238 significantly decreased serum immunoglobulin levels from mean pretherapy IgG2b levels of $5.9 \mathrm{mg} / \mathrm{ml}$ to $0.7 \mathrm{mg} / \mathrm{ml}$. While continued treatment resulted in sustained inhibition of tumor activity and growth of peripheral lymph nodes, analysis of terminal $\left[{ }^{18} \mathrm{~F}\right] \mathrm{FDG}-\mathrm{PET}$ images revealed a mean MLN SUVmax value of $2.4 \pm 0.7$ and thus indicated the MLN as a potential site of relapse upon acquisition of drug resistance. $\left[{ }^{18} \mathrm{~F}\right] \mathrm{FDG}-\mathrm{PET}$ was useful in objectively validating therapy responses in transgenic model of human multiple myeloma which can be correlated with serum immunoglobulin levels. Moreover, $\left[{ }^{18} \mathrm{~F}\right]$ FDG-PET was able to detect sites of tumor relapse which may be relevant for studies involving drug resistance.

\section{3}

Assessing the therapeutic and diagnostic potential of human thymidine kinase 1 in leukemic cell lines. D. Estevez, K.L. O'Neill; Brigham Young University, Provo, UT

Thymidine Kinase 1 (TK1), a salvage pathway enzyme responsible for recycling thymine in cells for use in DNA replication and repair, is normally tightly regulated by the cell cycle; however, research studies have shown that it is over-expressed and unregulated in cancer cells. Studies linking TK1 localization to the plasma membrane of lymphoma cells have suggested that this enzyme could also be used as a molecular target for cancer diagnosis and therapy. Previous research in our laboratory showed that TK1 located on the plasma membrane of different cancer cell lines had high enzymatic activity. This study was performed to support the eventual possibility of targeting TK1 for cancer diagnosis and immunotherapy in leukemias expressing high levels of TK1 on their plasma membrane. TK1 levels on plasma membrane were analyzed using BD FACSCanto flow cytometry on lymphoblastoid cells derived from Burkitt's lymphoma (Raji cells), acute T cell leukemia (Jurkat cells), myeloblastic cells derived from promyelocytic leukemia (HL-60 cells), and normal lymphocytes from healthy individuals. Analysis was performed on unpermeabilized cells in exponential growth phase using both a mouse monoclonal anti-TK1 $\mathrm{Ab}$ and a rabbit polyclonal anti TK-1 Ab. Cells were incubated with human FcR blocking reagent to eliminate non-specific binding to $\mathrm{Fc}$ receptors. Permeabilized cells were excluded using propidium iodide staining. Using an IgG Isotype control we were able to select proper gating ( $0 \%$ binding). TK1 surface expression was compared to expression of sodium potassium ATPase, a common plasma membrane marker, and pan-leukocyte marker CD45. Results confirmed high levels of TK1 expression on cancer cell lines analyzed. Flow cytometry analysis showed that binding of the mouse monoclonal anti-TK1 and rabbit polyclonal anti-TK1 was $93.4 \%$ $(\mathrm{SEM}=1.05, \mathrm{~N}=19)$ and $99.5 \%(\mathrm{SEM}=0.67, \mathrm{~N}=19)$ in Raji cells; $71.1 \%$ $(\mathrm{SEM}=3.41, \mathrm{~N}=19)$ and $89.4 \%(\mathrm{SEM}=2.74, \mathrm{~N}=19)$ in HL-60 cells; $63 \%$ $(\mathrm{SEM}=1.23, \mathrm{~N}=26)$ and $88.6 \%(\mathrm{SEM}=1.81, \mathrm{~N}=27)$ in Jurkat cells. Lymphoblastoid cells showed significantly higher TK1 expression when compared to that of lymphocytes obtained from healthy individuals: $9.9 \%$ $(\mathrm{SEM}=2.39, \mathrm{~N}=7)$ and $14.9 \%(\mathrm{SEM}=2.43, \mathrm{~N}=3)$. When induced with pokeweed mitogen there was no increase in TK1 expression on the plasma membrane of normal dividing lymphocytes: $7.9 \%(\mathrm{SEM}=0.54, \mathrm{~N}=3)$ and $10.63 \%(\mathrm{SEM}=2.89, \mathrm{~N}=3)$. Fluorescence microscopy observations provided more evidence of TK1 association with the plasma membrane of cancer cells. These results suggest that membrane-bound TK1 has potential for both diagnosis and immunotherapy in human leukemia.

\section{4}

Annotating oncogene status in castration-resistant prostate cancer with zirconium-89-labeled radiotracers. M.J. Evans, J.P. Holland, D. Ulmert,

*AACR-Aflac, Incorporated, Scholar-in-Training Award winner.
J.S. Lewis, C.L. Sawyers; Memorial Sloan-Kettering Cancer Center, New York, NY

As parallel advances in cancer biology and drug development continue to elevate the role of targeted therapies in oncology, the need for imaging biomarkers that systematically measure the biology associated with therapeutic intervention has become more urgent. Although the molecular imaging community has a commitment to develop technologies to this end, few investigational radiotracers directly measure the biology of common oncogenic signaling pathways often addressed by targeted therapies. We have developed a panel of radiotracers rationally designed to measure the pathobiology of two oncogenic drivers of prostate cancer, the androgen receptor and MYC. The common theme among these radiotracer development programs is that expression changes among "imageable" target genes can be exploited to non-invasively measure the pathological activation (and pharmacological inhibition) of the respective oncogenic transcription factors. Preclinical proof-of-concept studies underscore the remarkable promise of this model for radiotracer design, and early clinical translation is underway to validate the hypothesis that imaging molecular events more tightly regulated by oncogene signaling may increase the information content of PET scans in the context of treatment monitoring.

\section{$25^{*}$}

Mining cancer genomics data for identification of cancer stem cell targets. H. Fan-Minogue, J. Wheeler, A. Butte; Stanford University, Stanford, CA

Cancer stem cells (CSCs) are a small group of cancer cells with stem celllike properties and have been revealed to be the driving force of tumorigenesis, metastasis and drug resistance in many cancers. Although putative CSC surface biomarkers, such as CD133, CD44 and CD166, have been used to isolate CSC population, they are often not specific and unassociated with outcome. To identify CSC functional targets, we applied data mining approach to the lung cancer data from The Cancer Genome Atlas (TCGA). We systematically screened for genes whose expressions are statistically correlated with CSC surface biomarkers. Normalized DNA microarray data of 160 cases of squamous carcinoma and 33 cases of adenocarcinoma and 8 cases of normal lung were analyzed for association with CD133, CD44 and CD166 respectively. Multiple hypothesis testing was corrected with the false discovery rate (FDR) less than 1\%. We found CD133, CD44 and CD166 each has a different set of statistically associated genes in either adeno or squamous subtypes. Moreover, correlated genes with each of the surface markers are different between adeno and squamous subtypes. These distinctive expression patterns imply that each surface biomarker involves different signaling pathways and they are differentially regulated between subtypes of lung cancer. We also applied gene ontology to enrich for cell surface targets that are accessible for extracellular molecular probes. Among the most correlated receptor genes with $\mathrm{CD} 133$ in squamous subtype is a hormone receptor of the reproduction regulator oxytocin (OXTR, $\mathrm{r}=0.4276, p=1.699 \mathrm{e}-8)$. OXTR has recently been shown to be involved in tumor cell growth and has many clinically used antagonists for reproduction regulation. Our work suggests great heterogeneous molecular features of CSC in different cancer types. We also show that an association study of CSC surface markers can be a useful way to identify functional CSC biomarkers, which can be potentially used for development of probes for CSC imaging and targeting.

\section{6}

Imaging therapeutic response using RECIST criteria: Y-90 SIRSpheres treatment of unresectable liver tumors. Z. Zhang, M.R Fardanesh, J. Machac, S. Heiba, K. Knesaurek, I. Oyfe, R. Lookstein, S. Nowakowski, A. Fischman, E. Kim, V. Zaretsky, A. Mihaila; Mount Sinai School of Medicine, New York, NY

Objectives: To evaluate the efficacy of Y-90 SIR-Spheres treatment in patients (pts) with unresectable liver tumors using RECIST criteria. Methods: 79 pts with unresectable primary or metastatic liver tumors: neuroendocrine (NET) (34), hepatocellular carcinoma (HCC) (26), and other tumors (26) underwent 86 Y-90 SIR-Spheres treatments. Tc-99m MAA imaging was used to evaluate liver arterial perfusion and lung 
shunting (LS). The treatment response was assessed by CT and/or MRI using RECIST criteria. Results: The median absorbed doses for the tumor and liver were 56.9 Gy and 19.5 Gy, respectively. The median LS was $4.0 \%$. 27 treatments were injected with calculated standard doses, and 59 doses were reduced by $25 \%-40 \%$. 2 treatments $(2.3 \%)$ had complete responses (CR), $32(37.2 \%)$ had partial response (PR), 28 (32.6\%) had stable disease (SD), and 24 (27.9\%) had progressive disease (PD). Pts with NET showed a higher positive response $(\mathrm{PR}+\mathrm{CR})$ rate $(51.5 .0 \%)$ compared to pts with HCC (38.5\%) and other tumors (26.9\%), pts with HCC showed a higher PD rate $(40 \%)$ compared to pts with NET $(20.6 \%)$ and other tumors $(26.9 \%)$, but they are not statistically significant. There was no significant response difference between pts treated with calculated standard dose vs. reduced dose. 28 infusions only delivered $30 \%-90 \%$ of prescribed dose due to flow stasis, but stable disease and partial response were still observed in 6 and 16 infusions, respectively. There was no radiation hepatitis and treatmentinduced liver failure observed after the treatments. One patient had gastric ulceration and was managed conservatively. Conclusions: These results suggest Y-90 SIR-Spheres treatment is a useful modality with a remarkable objective response rate and with acceptable toxicity for the treatment of pts with unresectable liver tumors.

\section{7}

Rapid diagnosis of human astrocytic tumors using sulforhodamine 101. J.F. Georges ${ }^{1}$, N.L. Martirosyan ${ }^{1}$, J. Eschbacher ${ }^{1}$, J. Nichols ${ }^{2}$, M. Tissot ${ }^{3}$, A.M. Elhadi ${ }^{1}$, G. Mendes ${ }^{1}$, M. McQuilkin ${ }^{3}$, B.G. Feuerstein ${ }^{2}$, R.F. Spetzler ${ }^{1}$, T. Anderson ${ }^{2}$, M. Preul ${ }^{1}$, P. Nakaji ${ }^{1}$; ${ }^{1}$ Barrow Neurological Institute, Phoenix, AZ, ${ }^{2}$ University of Arizona College of Medicine, Phoenix, AZ, ${ }^{3}$ Arizona State University, Tempe, AZ

Introduction: Surgical resection of brain tumors is guided by intraoperative diagnostics. However, frozen section analysis, the method for obtaining these diagnoses, lacks the specificity to effectively differentiate some tumors with conflicting treatment plans (ie, astrocytoma and lymphoma). When specificity is required, clinical teams must typically wait 24-72 hours for a diagnosis from post-operative antibody staining of biopsied tissue. This timeframe precludes intraoperative feedback, and can result in additional surgery for a patient if tumor resection is prematurely halted. Here we show that live cell imaging of fresh human brain tumor biopsies rapidly labeled with the physiological fluorophore Sulforhodamine 101 (SR101) can rapidly differentiate astrocytic tumors and reactive astrocytes from non-astrocytic tumors and normal brain. Furthermore, this technique can provide antibody-like specificity in a time frame that supports intraoperative decision-making. Methods: Human astrocytoma cells were cultured on glass-bottom dishes $(\mathrm{n}=5)$, incubated with SR101, and live cells imaged with a Zeiss LSM710 confocal microscope. Acute slices from human glioma $(\mathrm{n}=8)$ and human lymphoma $(\mathrm{n}=6)$ orthotopic xenografts were incubated with SR101 or its fixable analog and imaged with confocal microscopy. Glioma and lymphoma slices ( $\mathrm{n}=18$ slices) were respectively counter-immunostained with GFAP and CD20 for stereological assessment of SR101 co-localization. Additionally, sixty-four undiagnosed fresh human brain tumor biopsies were rapidly incubated with SR101, imaged, and compared to final pathological diagnosis. Results: SR101 rapidly filled the cytoplasm of human astrocytoma cells in culture and in some cases delineated cell nuclei. SR101 morphologically differentiated astrocytic tumors from reactive astrocytes. In acute slices, SR101 labeled $86.53 \%$ of astrocytoma cells and $2.17 \%$ of lymphoma cells. In human biopsies, SR 101 rapidly labeled reactive astrocytes and selectively identified 12 of 13 astrocytomas. In non-astrocytic tumors, SR101 labeled cells that resembled reactive astrocytes but not tumor cells. SR101 did not label CNS lymphoma or the majority of oligodendroglioma biopsies. SR101 labeling and imaging of human tissue could be completed in under 15 minutes. Conclusion: We have demonstrated a technique for identifying the most common primary brain tumor, astrocytoma, by ex vivo exposure to the fluorescent dye SR 101 . SR101 can rapidly and selectively label human astrocytic tumors and astrocytes in a time frame that supports intraoperative decision-making. This is the first reported use of a functional dye on living human brain tumor tissue to provide a clinically meaningful immediate histopathological diagnosis. We believe this is only the first venture into a new area of advanced intraoperative molecular imaging diagnostics. Application of this technique to other pathologies and organ systems will advance as new targeted fluorophores are identified and developed.
Ultrashort TE imaging with SPIONs: bright prospects for in vivo applications. C.A. Gharagouzloo ${ }^{1}$, S. $\mathrm{Madi}^{2}$, R.T. Seethamraju ${ }^{3}, \mathrm{M}$. Harisinghani ${ }^{4}$, S. Sridhar ${ }^{1}$; ${ }^{1}$ Northeastern University, Boston, MA, ${ }^{2}$ Bruker BioSpin Corp., Billerica, MA, ${ }^{3}$ Siemens Medical Solutions, USA Inc., Boston, MA, ${ }^{4}$ Radiology, Massachusetts General Hospital, Boston, MA

Introduction: Conventional $\mathrm{T} 2$ and $\mathrm{T} 2 *$ weighted imaging techniques manifest signal voids for SPION-containing media. This proves to be disadvantageous in most circumstances because of the difficulty in discriminating signal loss from tissue associated partial voluming, perivascular effects, susceptibility artifacts and motion or flow artifacts. The combination of ultra-short TE (UTE) and SPIONs, however, produces high SNR and bright contrast images with specific advantages, such as biocompatibility, long blood half-life and in vivo quantifiability, that could pave a road for magnetic nanoplatforms as clinically effective contrast agents (CA) in the near future. Ferumoxytol is currently the only magnetic nanoplatform that is FDA approved and commercially available, albeit its approval is for iron deficiency anemia (IDA). The majority of clinical trials utilize standard $\mathrm{T} 1$ and $\mathrm{T} 2$ imaging sequences for prostate cancer and gliomas and also include standard MR angiography (1), though clinical work involving positive contrast is under way (2). Method: UTE 3D Optimization: UTE was optimized on a Bruker 7T Biospec at the Center for Translational Neuroimaging (CTNI) at Northeastern University. High-contrast images were obtained by modification of various imaging parameters, such as TE, TR, flip angle, pulse length, polar under-sampling, bandwidth and FOV/ geometry. We generally use phantoms of $5 \mathrm{mM} \mathrm{CuSO} 4$ to pre-calculate protocol trajectories. Our initial experience with fat suppression helped to accentuate SPION-containing media; however, the accompanied trade-off was a significant increase in imaging time. We determined that with a high enough flip angle ( $30-45$ degrees) fat could be suppressed enough for high-contrast images without the need for fat suppression. Optimization included a TE of $\sim 30 \mu$ s and a TR of $6 \mathrm{~ms}$. High resolution 3D images $(\sim 200 \mu \mathrm{m})$ can be taken over a region of $40 \times 40 \times 40 \mathrm{~mm}$ in about 5 minutes. Ferumoxytol nanoparticles: Ferumoxytol consists of $\mathrm{Fe} 3 \mathrm{O} 4$ of $<10 \mathrm{~nm}$ diameter SPIONs that are dextran surface functionalized. They have a long blood circulation half-life of 16 hours. Their hydrodynamic diameter is $26 \mathrm{~nm}$. Results: In vitro results of ferumoxytol-doped media showed a linear relaxivity r2 (slope of [Cspions] vs. R2) contingent on media (water, 1\% agar, 2\% agar). For media with inherent high $\mathrm{R} 2[\mathrm{C}]=0, \mathrm{r} 2$ was lower, such that each media converged on the same value of R2. After linear convergence at around $100 \mu \mathrm{g} / \mathrm{ml}$, phantoms became dim and then eventually completely dark. After this concentration, R2 not only fell off but was dominated by SPION concentration and no longer showed a media dependent difference. Pre-contrast in vivo results in nude mice show that signal almost uniquely comes from the stomach and intestines, which have bright contrast. Blood and other tissue are dark and approach background noise as the flip angle increases. A high flip angle therefore saturates tissue and can be used instead of fat suppression to have time-efficient, high-contrast images. $4 \mathrm{H}$ post injection of ferumoxytol shows angiographic type images because of the long half-life $(16 \mathrm{H})$ of the product. After $40 \mathrm{H}$, ferumoxytol is still present in the animal, and depending on the initial injection concentration, the contrast changes in accordance with something similar to the in vitro results. Conclusion: The combination of UTE and SPIONs holds a bright future, inasmuch as the benefits of positive contrast and biocompatibility come together. Currently ferumoxytol remains the arrowhead for immediately applicable clinical studies because of FDA approval. We are currently pursuing ferumoxytol as well as other magnetic nanoplatforms for in vivo applications. Acknowledgments: Disclosure of author financial interest or relationships: We acknowledge partial support from NSF DGE 0965843, HHS/5U54CA151881-02, and the Electronics Materials Research Institute at Northeastern University. References: 1. Ferumoxytol use as an intravenous contrast agent for magnetic resonance angiography, Katie L Stabi and Lisa M Bendz, The Annals of Pharmacotherapy, 2011. 2. Positive contrast imaging with Feraheme UTE or IRON for clinical application, Ravi T. Seethamraju et al., WMIC poster, 2011. 
A theranostic approach to the management of pancreatic ductal carcinoma (PDC). D.M. Goldenberg ${ }^{1}$, D.V. Gold ${ }^{1}$, R.M. Sharkey ${ }^{2}$, W.J. $\mathrm{McBride}^{2}$, A.J. Ocean ${ }^{3}$, H. Horne ${ }^{2}$, W.A. Wegener ${ }^{2} ;{ }^{1}$ Garden State Cancer Center, Center for Molecular Medicine and Immunology, Morris Plains, NJ, ${ }^{2}$ Immunomedics, Inc., Morris Plains, NJ, ${ }^{3} \mathrm{New}$ York Presbyterian HospitalWeill Cornell Medical College, New York, NY

Objective: Using the PAM4 monoclonal antibody that is highly specific for PDC, we developed a sensitive and specific enzyme immunoassay (EIA) for detecting the antigen biomarker in blood, a PAM4-based immunoPET imaging agent, and a therapeutic comprising humanized PAM4 (hPAM4) conjugated with the beta-emitter, ${ }^{90} \mathrm{Y}\left({ }^{90} \mathrm{Y}\right.$ clivatuzumab tetraxetan). Our purpose is to describe how these can be interactive to provide a personalized theranostic approach to the management of patients with PDC. Methods and Results: An EIA based on the PAM4 antibody has been validated in over 600 serum samples for detection of PDC, with PAM4 antigen being detected in $71 \%$ of confirmed early-stage disease, and $91 \%$ in patients with advanced disease. In specimens from patients with benign pancreatic disease, specificity was $81 \%$. A total of 126 specimens from patients with benign disease, including 80 from patients with chronic pancreatitis (CP), were evaluated. Overall, $24(19 \%)$ were positive for the PAM4 antigen, with the majority found in the CP patients [ 18 of 80 positive $(23 \%)$ ], all of whom had surgical resection performed for severity and/or duration of disease activity. Tissue staining of CP tissues has demonstrated that PAM4 does not react with inflamed parenchyma, but rather with occult malignancy and/or benign neoplastic precursor lesions (PanINs) commonly associated with CP. Thus, the PAM4 assay may be detecting early signs of PAC in some portion of the $\mathrm{CP}$ patients. A bispecific antibody (bsMAb) construct consisting of 2 binding arms of PAM4 and one binding arm for a hapten-peptide has been developed for pretargeting radionuclides for PET imaging. The pretargeting method is a highly sensitive procedure for localizing tumors, and when paired with the PAM4 antibody, provides a highly selective targeting method for detecting PDC. For example, tumor/blood ratios in mice bearing human PDC xenografts pretargeted with the PAM4 bsMAb and an ${ }^{111}$ In-labeled hapten-peptide were $>2000: 1$ within $3 \mathrm{~h}$. Finally, the hPAM4 antibody labeled with ${ }^{90} \mathrm{Y}$ has been studied combined with gemcitabine in a Phase I/II trial that included 100 patients in the first-line setting. The combination, which comprised a fractionated dosing regimen, with each cycle consisting of weekly x 4 gemcitabine (200 to $1000 \mathrm{mg} / \mathrm{m}^{2} /$ dose) and weekly x $3{ }^{90} \mathrm{Y}$ clivatuzumab tetraxetan $\left(6.5\right.$ to $\left.15 \mathrm{mCi} / \mathrm{m}^{2} / \mathrm{dose}\right)$, showed (i) improved therapy as the ${ }^{90} \mathrm{Y}$ dose was escalated, (ii) myelosuppression was the major, but reversible, toxicity, (iii) overall survival (OS) improved with repeated vs. single dosing cycles for low-dose gemcitabine (median OS: 11.8 vs. 5.4 months), but increasing the gemcitabine dose above $200 \mathrm{mg} / \mathrm{m}^{2} \times 4$ did not improve outcome (final median OS: 9.3 vs. 5.2 months, respectively). Currently, a 2 -arm trial is evaluating repeated cycles of the combination therapy vs. ${ }^{90} \mathrm{Y}$ clivatuzumab tetraxetan alone in advanced PDC patients with at least 2 prior therapies. Conclusions: Using a PDC-specific antibody has allowed for: (i) EIA tracking of a serum biomarker as a monitoring and diagnostic tool, (ii) a diagnostic imaging approach to better define sites of disease and response to therapy, and (iii) a radioimmunotherapy agent for use alone or in combination with gemcitabine or possibly other therapeutic modalities.

\section{0}

Cancer diagnosis characterization and treatment: a new paradigm. B.S. Greenspan; GHSU, Augusta, GA

The current standard of care for therapy of cancer - surgery, chemotherapy, and radiation therapy - is unsatisfactory for many types of cancer. While results for many childhood cancers, especially leukemia, are fairly good, survival rates for many of the more common adult malignancies are not especially good. Also, while it is known that various metastatic sites often contain different clones of cells, there is currently no provision to address this. Worse, many of the current chemotherapy treatments have significant unpleasant side effects. Therefore, I propose a modern comprehensive approach to cancer diagnosis, characterization (including staging) and therapy, based on the molecular biology of cancer. This approach will improve characterization of tumors, and lead to more specific, targeted personalized therapy that will produce significant improvement in survival, hopefully with fewer adverse side-effects. This approach is based in part on the work of Hanahan and Weinberg. They described six hallmarks of cancer in their seminal article of 2000. An updated article in 2010 discussed advances since the original article, and added two more hallmarks. These eight hallmarks are: 1) Self-sufficiency in growth signals (sustaining proliferative signaling), 2) Evading growth suppressors, 3) Evading apoptosis, 4) Enabling replicative immortality, 5) Sustained angiogenesis, 6) Activating tissue invasion and metastasis, 7) Reprogramming of energy metabolism, 8) Evading immune destruction. Underlying the above hallmarks, according to Hanahan and Weinberg, are genome instability and inflammation. I suggest the following: For all of these imaging studies, PET is used as PET/CT. PET/MR may be useful as well in certain circumstances. For diagnosis and identification of various cancers, PET/CT with F-18 FDG is currently used. In addition, other PET agents, also with PET/CT, to more specifically identify various tumors by proliferation, cell-surface receptors, or other specific features of these tumors. For characterization, including staging and determination of different clonal types: evaluation by several different PET radiopharmaceuticals (agents) in addition to F-18 FDG. 1. Proliferation agents to assess DNA synthesis (e.g., F-18 FLT, F-18 ISO [a sigma-2 receptor agent]); 2. Agents to assess amino acid synthesis (e.g., C-11 methionine, $\mathrm{C}-11$ or $\mathrm{N}-13$ glutamine); 3 . Receptor agents to evaluate the presence and amount of various cell-surface receptors; 4. Hypoxia agents (e.g., F-18 FMISO, Cu-64 ATSM); 5. Angiogenesis agents (e.g., Cu-64 RGD); 6. Other agents as appropriate (evaluation of osteoclastic activity, for instance); 7. PET agents to specifically evaluate each of the 8 hallmarks listed above. The goal is to not only make a diagnosis, such as lung cancer, or breast cancer, but utilize this advanced imaging to determine a subtype. For characterization, determine staging and location of metastatic disease, and identify the characteristics of each clone of cells present in each metastatic deposit. This advanced imaging will identify not only the location of metastases, but will characterize all of the lesions, which will lead to more specific and more comprehensive targeted therapies. For therapy: 1) Model: I-131 therapy of thyroid cancer (this utilizes the negative feedback loop of TSH - T4/T3, where TSH stimulates synthesis of T4, with subsequent peripheral conversion to $\mathrm{T} 3$, but increasing $\mathrm{T} 4$ levels suppress production of TSH). I have not seen this model as a basis for cancer therapy published anywhere. 2) Whole body radiation by radiation oncology: I propose initially $100 \mathrm{rem}$ whole body exposure, to provide immune stimulation (Dr. Myron Pollycove, personal communication). This idea is known by radiation oncologists, and the ultimate administered level of radiation may be different. 3) Disrupt the signaling pathways in each tumor specific for each patient of these 8 hallmark cell functions, utilizing the thyroid cancer model and PET agents as appropriate. This approach would require evaluation of enzymatic pathways for targeting for each tumor of the various hallmarks (as many as possible). For these hallmarks, identify enzymatic pathways that are already upregulated or could be upregulated, and then shut off, with the use of diagnostic/therapeutic (theranostic) pairs of labeled agents, utilizing chemical antagonists for these enzymatic pathways and also radionuclides for therapy. Ideal pairs would be of the same element, but other pairs may work as well. For example, use of I-124 for imaging and I-131 for therapy to identify and target various pathways. Another example is use of $\mathrm{Cu}-64$ labeled agent to identify upregulated receptors, and then give a $\mathrm{Cu}-67$ labeled agent for therapy. Identifying and targeting enzymatic pathways for as many of these hallmarks as possible in each tumor, and being able to target specific clones of tumor cells in each metastatic deposit, may be able to stop or significantly slow tumor progression. 4) Disrupt the contribution of the supporting structure, including recruitment of normal cells that surround metastatic deposits. Disrupting the tumor microenvironment may have significant beneficial effects. 5) Disrupt the inflammatory process surrounding metastatic deposits. I believe that this comprehensive, combinatorial, targeted approach will produce much better outcomes, and do so with significantly fewer side effects. The goal is to improve the quality of life and survival.

\section{1}

Tumor paint provides near-infrared fluorescence detection of tumor tissue in mouse models of skin cancer. C.G. Hubert ${ }^{1}$, S. Hansen ${ }^{2}$, M. Stroud $^{2}$, J. Parrish-Novak ${ }^{2}$, J. Olson ${ }^{1}$; ${ }^{1}$ Fred Hutchinson Cancer Research Center, Seattle, WA, ${ }^{2}$ Blaze Bioscience, Inc., Seattle, WA

For many types of cancer, surgery remains a primary treatment modality and the extent of surgical resection is directly related to survival. Depending 
on tumor type and stage, complete resection can improve overall survival by $30-60 \%$. Tumor Paints are being developed as real-time intraoperative guides for cancer surgeries. The first Tumor Paint being developed is a conjugate of a chlorotoxin (CTX) peptide and a near-infrared fluorophore. Use of this Tumor Paint during resection has the potential to assist surgeons in obtaining negative tumor margins and sparing normal surrounding tissues. Its targeting peptide, based on chlorotoxin, provides specificity for cancer cells. The near-infrared fluorescent tag is taken up by the cancer cells along with the peptide, producing a long-lasting signal that can be detected days after administration. This enables flexibility in time of delivery of the drug prior to surgery. The broad tumor recognition of the CTX peptide justifies initial clinical trials in a variety of solid tumor types. Monitoring of near-infrared fluorescence over the $\sim 24$ hours between drug administration and surgery would provide valuable information on the relationship between time of administration and signal-to-background ratio. Efficacy in cancers that are accessible to non-invasive imaging, such as skin cancers, would enable this objective. In order to demonstrate effective imaging of skin cancers, the candidate Tumor Paint was tested in mouse models of amelanotic skin cancers (basal cell carcinoma (BCC), squamous cell carcinoma (SCC), and amelanotic melanoma). K5-Gli2:C57bl/6 albino mice (BCC model) were obtained from Dr. Allan Oseroff at the Roswell Park Cancer Institute. Pold $1 D^{400 A / D 400 A}:$ FVB mice (SCC model) were obtained from Dr. Bradley Preston at the University of Washington. Amelanotic melanoma xenografts were prepared by injecting $10^{6} \mathrm{~A} 375$ cells into the flanks of $\mathrm{Nu} / \mathrm{Nu}$ mice. The Tumor Paint ( $2 \mathrm{nmole}$ ) was administered to 3 tumor-bearing and 2 control mice for each model via tail vein injection. Mice were imaged 3-4 days later using a Xenogen Spectrum imaging system. Mice were euthanized, and tumor-bearing tissues were imaged separately and cancer specificity assessed histologically. Cancerous foci were visible on the ears of $3 \mathrm{~K} 5$-Gli2 mice and 2 Pold $1 D^{400 A / D 400 A}$ mice. Near-infrared imaging showed staining in $5 / 5$ ears with tumors, and in A375 flank tumors. Mice that did not have visible lesions did not show specific fluorescence. $\mathrm{H} \& \mathrm{E}$ staining showed cancerous histopathologic changes corresponding to the Tumor Paint signal. These studies demonstrate that non-melanotic skin cancers can be recognized and illuminated using a Tumor Paint. This preclinical study supports future evaluation of Tumor Paints in these indications for resection, margin evaluation, and perhaps screening in high risk individuals.

\section{$32^{*}$}

The functional role of perivascular macrophages in the tumor microenvironment of metastasis. A.S. Harney, E.N. Arwert, D. Entenberg, Y. Wang, J.S. Condeelis; Albert Einstein College of Medicine of Yeshiva University, Bronx, NY

In mammary tumors a subpopulation of cancer cells intravasate when associated with perivascular macrophages. These migratory tumor cells over-express Mammalian enabled (Mena), a family of proteins that function at the convergence of motility pathways controlling actin polymerization, directional cell movement toward EGF, and invadopodium formation. The microanatomical sites of tumor cell intravasation consisting of a perivascular macrophage interacting with a Mena over-expressing tumor cell have been named "tumor microenvironment of metastasis" (TMEM) and their density is correlated with metastatic breast cancer in patients. In spontaneous orthotopic mouse mammary tumors where the expression of the polyoma middle $\mathrm{T}$ antigen (PyMT) is under the control of the mouse mammary tumor virus (MMTV), as the tumor progresses to malignancy tumor cells have increasingly elevated Mena expression and assemble TMEM. TMEM assembly is correlated with both elevated levels of circulating tumor cells and the number of lung metastases, indicating TMEM has a functional role in tumor cell dissemination. Since perivascular macrophages have been shown to be present at sites of hematogenous dissemination of tumor cells, ongoing work aims to understand the functional role of perivascular macrophages in the intravasation activity of the TMEM compartment. Using high-resolution multiphoton-based live animal time-lapse microscopy the association of a tumor cell and a macrophage on a blood vessel as a TMEM in a live animal is shown to be a stable structure over time. The dynamics of the interactions between perivascular macrophages and tumor cells and between perivascular macrophages and endothelial cells are

*AACR-Aflac, Incorporated, Scholar-in-Training Award winner. studied using high-resolution multiphoton intravital imaging. Perivascular macrophages mediate tumor cell intravasation, and an understanding of the mechanism by which this unique subset of tumor associated macrophages adhere to and interact with the endothelium is under investigation.

\section{3}

Novel probe for noninvasive detection of tumor-associated inflammation. A. Balducci ${ }^{1}$, Y. Wen ${ }^{2}$, Y. Zhang ${ }^{2}$, B.M. Helfer ${ }^{1}$, T.K. Hitchens ${ }^{3}$, W.S. Meng ${ }^{2}$, J. Janjic ${ }^{2}$, A.K. Wesa ${ }^{1} ;{ }^{1}$ Celsense Inc., Pittsburgh, PA, ${ }^{2}$ Duquesne University, Pittsburgh, PA, ${ }^{3}$ Carnegie Mellon University, Pittsburgh, PA

A novel, dual-mode contrast agent was formulated through the addition of an optical near infrared (NIR) probe to a perfluorocarbon (PFC)-based ${ }^{19} \mathrm{~F}$ magnetic resonance imaging (MRI) agent, which labels inflammatory cells in situ. A single PFC-NIR imaging agent enables both qualitative, rapid optical monitoring of an inflammatory state, and detailed, quantitative, and tissue-depth independent magnetic resonance imaging (MRI). The feasibility of in vivo optical imaging of the inflammatory response was demonstrated in a subcutaneous murine breast carcinoma model. Ex-vivo optical imaging was used to quantify the PFC-NIR signal in the tumor and organs, and results correlated well to quantitative ${ }^{19} \mathrm{~F}$ NMR analysis of intact tissues. ${ }^{19} \mathrm{~F}$ MRI was employed to construct a three-dimensional image of inflammatory cell distribution at the tumor site. Flow cytometry of tumor isolates was used to identify the cellular localization of the PFC-NIR within the tumor microenvironment. Results show that imaging contrast is enhanced through labeling of host cells involved in the immune response, but not tumor cells. The major cellular reservoir of the imaging agent was found amongst $\mathrm{CD} 11 \mathrm{~b}+\mathrm{F} 4 / 80 \mathrm{low}$ Gr-1low infiltrating cells, which represents a cellular phenotype consistent with myeloid-derived suppressor cells (MDSC), which are implicated in immune evasion and tumor progression. Using this PFC-NIR contrast agent with non-invasive, quantitative imaging techniques could serve as a valuable tool in for evaluating novel anti-cancer agents.

\section{4}

In vivo imaging of cell-cycle phase of individual cancer cells throughout growing tumors in live mice. S. Yano ${ }^{1}, \mathrm{Y}$. Tome ${ }^{1}$, M.A. Digman ${ }^{2}, \mathrm{M}$. Momiyama $^{1}$, A. Suetsugu ${ }^{1}$, H. Kishimoto ${ }^{3}$, H. Tazawa ${ }^{4}$, T. Fujiwara ${ }^{3}$, E. Gratton ${ }^{2}$, R.M. Hoffman ${ }^{1}$; ${ }^{1}$ AntiCancer Inc., San Diego, CA, ${ }^{2}$ Department of Biomedical Engineering, Physics, and College of Medicine, University of California, Irvine, CA, ${ }^{3}$ Department of Gastroenterological Surgery, Okayama University Graduate School of Medicine, Dentistry and Pharma Sci, Okayama, Japan, ${ }^{4}$ Center for Innovative Clinical Medicine, Okayama University Hospital, Okayama, Japan

The phase of the cell cycle can determine whether a cancer cell can respond to a given drug. This report presents imaging methodology to obtain realtime cell cycle information on cancer cells throughout tumors in live mice using a fluorescence ubiquitination cell cycle indicator (FUCCI). In nascent tumors in nude mice, approximately $30 \%$ of the cells in the center of the tumor were in $\mathrm{G}_{0} / \mathrm{G}_{1}$ and $70 \%$ in $\mathrm{S} / \mathrm{G}_{2} / \mathrm{M}$. In contrast, approximately $90 \%$ of cancer cells in the center of an established tumor were in $\mathrm{G}_{0} / \mathrm{G}_{1}$ phase. Similarly, approximately $75 \%$ of cancer cells far from $(>100 \mu \mathrm{m})$ tumor vessels were in $\mathrm{G}_{0} / \mathrm{G}_{1}$. Although $60-70 \%$ of cancer cells near the surface in an established tumor $(<100 \mu \mathrm{m})$ or near blood vessels were in $S / G_{2} / M$, these cells are a small minority of an established tumor. Moreover, static tumors in mice consisted of mostly $\mathrm{G}_{0} / \mathrm{G}_{1}$ cells, suggesting they are dormant. Real-time imaging of the cancer cell cycle has profound implications for cancer therapy, since most drugs currently in use target cells in $\mathrm{S} / \mathrm{G}_{2} / \mathrm{M}$, which are a minor fraction in an established tumor. With the imaging method developments reported here, drugs that target non-dividing cancer cells can be readily identified.

\section{5}

S. typhimurium A1-R tumor-targeting and efficacy in immunocompetent mice potentiated by primer dosing. Y. Tome ${ }^{1}, \mathrm{Y}$. Zhang ${ }^{2}, \mathrm{M}$. Momiyama ${ }^{2}$, K. Tomita ${ }^{3}$, H. Tsuchiya ${ }^{3}$, M. Bouvet ${ }^{4}$, R.M. Hoffman ${ }^{2}$, M. Zhao'; ${ }^{1}$ Department of Orthopedic Surgery, Graduate School of Medicine, University of the Ryukyus, Okinawa, Japan, ${ }^{2}$ AntiCancer Inc., San Diego, CA, ${ }^{3}$ Department of Orthopedic Surgery, Graduate School of Medical Science, 
Kanazawa University, Kanazawa, Japan, ${ }^{4}$ Department of Surgery, University of California, San Diego, CA

We have previously developed the tumor targeting strain Salmonella typhimurium $\mathrm{A} 1-\mathrm{R}(\mathrm{A} 1-\mathrm{R})$ and have shown it to be active against a number of tumor types in metastatic orthotopic nude mouse models. However, in immunocompetent mice, dosing of A1-R has to be adjusted to avoid toxicity. In the present study, we developed a strategy to maximize efficacy and minimize toxicity for A1-R tumor-targeting in immunocompetent mice implanted with Lewis lung carcinoma expressing RFP. A small primer dose of S. typhimurium A1-R expressing GFP was first administered $\left(1 \times 10^{6} \mathrm{cfu}\right.$ i.v.) followed by a high dose $\left(1 \times 10^{7}\right.$ cfu i.v. 4 hours later. GFP expression enabled in vivo imaging of A1-R. The primer-dose strategy resulted in smaller tumors and no observable side effects compared to high dose alone treatment. RFP expression enabled in vivo imaging of tumor growth. The serum level of TNF- $\alpha$ was elevated in the mice treated with the primer-dose compared to mice only given the high dose. Tumor vessel destruction was enhanced by primer dosing of $S$. typhimurium A1-R in immunocompetent mice expressing nestin-driven green fluorescent protein (ND-GFP), which is selectively expressed in nascent blood vessels. GFP expression enabled in vivo imaging of the tumor vessels. The primer dose may activate TNF- $\alpha$ and other cytokines in the mouse necessary for bacterial invasion of the tumor thereby allowing a subsequent therapeutic dose to be effective and safe.

\section{6}

Subcellular imaging of the efficacy of temozolomide on cancer cells in the brain of live mice. M. Momiyama ${ }^{1}$, A. Suetsugu ${ }^{1}, H$. Kimura ${ }^{2}, H$. Kishimoto $^{3}$, R. Aki ${ }^{1}$, T. Chishima ${ }^{4}$, M. Bouvet ${ }^{1}$, I. Endo ${ }^{4}$, R.M. Hoffman ${ }^{1}$; ${ }^{1}$ AntiCancer Inc., San Diego, CA, ${ }^{2}$ Department of Orthopedic Surgery, Graduate School of Medical Science, Kanazawa University, Kanazawa, Japan, ${ }^{3}$ Department of Gastroenterological Surgery, Okayama University Graduate School of Medicine, Dentistry and Pharma Sci, Okayama, Japan, ${ }^{4}$ Department of Gastroenterological Surgery, Yokohama City University, Yokohama, Japan

The efficacy of TMZ on cancer cells in the brain was determined by observation of tumor colony growth inhibition over time through a craniotomy open window in nude mice. Individual apoptotic dual-color U87 and LLC cells, expressing GFP in the nucleus and RFP in the cytoplasm, were observed through the craniotomy open window 10 days after treatment with TMZ (100 mg/kg i.p. for 5 consecutive days). Dual-color U87 cells with fragmented nuclei were visualized. GFP-expressing apoptotic bodies and destruction of RFP-expressing cytoplasm were also visualized. In addition, the terminal deoxynucleotidyltransferase-mediated deoxyuridine triphosphate nick-end labeling (TUNEL) assay was used to confirm apoptosis visualized by imaging the behavior of GFP-labeled cancer-cell nuclei. Dark-brown stained U87 nuclei, indicating apoptosis with the TUNEL assay, were detected in the brain tumor in mice which were treated with TMZ. Tumor volume in the treated group was significantly smaller than in the control group (at day 19, $p<0.001$ ). Subcellular imaging in live mice is a powerful tool to image anticancer drug efficacy.

\section{7}

A biophysical model of tumor cell proliferation and angiogenesis based on in vivo imaging data. D.A. Hormuth, II, T.E. Yankeelov; Vanderbilt University Institute of Imaging Science, Nashville, TN

Introduction: The availability of measurements describing the molecular, cellular, and physiological characteristics of tumors provided by noninvasive imaging has increased in recent years. In particular, MRI and PET have matured to the point where they can provide noninvasive, quantitative, and 3D characterizations of, for example, blood flow, vessel permeability, blood volume, cellularity, hypoxia, metabolism, and cell proliferation. One way to capitalize on the wealth of available information is to incorporate it into a realistic biophysical model of tumor growth that can be used to predict tumor growth and therapy response on an individual basis. The mathematical modeling literature typically models tumor growth by developing systems of equations describing, for example, chemotaxis, haptotaxis, and growth factor gradients which are extraordinarily difficult to obtain in an intact organism. By introducing imaging data into these models, terms requiring information available typically only by highly invasive methods (surgery, biopsy, or animal sacrifice) or within idealized (in vitro) systems can be replaced with terms supported by imaging data becoming a more applicable model to both pre-clinical and clinical studies. We (1-3) and others (4-6) have made some progress in this direction. Methods: Six Sprague-Dawley rats with C6 gliosarcomas were imaged with diffusion weighted MRI (DW-MRI) and dynamic contrast enhanced MRI (DCEMRI). Rats were imaged on days $9,10,11,13,15$, and 17 post tumor implantation. Cell number was estimated by ADC maps, calculated from DW-MRI, as previously described (1). ADC maps were also used to calculate tumor cell diffusion and proliferation maps as described by Ellingson et al. (7). Imaging data within the tumor was then incorporated into a model consisting of two coupled partial differential equations describing tumor cell number and glucose concentration solved using the finite difference method. At each time step tumor cells can move (randomly or directed) or proliferate in response to the spatial distribution of glucose and vascular status. A uniform vascular volume (3.4\% (8)) was assumed for the healthy brain whereas, in the tumor, $v_{p}$ (from DCE-MRI) provided this information. Simulations were initialized with data from day 9 (cell number and $v_{p}$ ) and proliferation and diffusion maps derived from days 9,10 , and 11. The agreement between predicted and observed tumor growth, as described by cell number and tumor volume, was assessed by the Pearson correlation coefficient (PCC) and the concordance correlation coefficient (CCC). Results: Predicted and observed cell number and tumor volume resulted in PCCs of .991 and .987 and CCCs of .645 and .735. These results suggest a strong linear relationship between predicted and observed values. Thus, the simulated spatial pattern and distribution matches that of the experimental data, though there is a systematic underestimation. Importantly, the predicted tumor resulted in a necrotic region which was also found in the experimentally measured tumor. We posit that incorporating angiogenesis into the model might more accurately reflect experimentally observed morphology. Conclusions: Preliminary results indicate the potential to predict in vivo tumor growth based off of imaging data obtained early in a tumor's growth cycle. It may be possible to use such an approach to develop a tumor "forecast" that can be used to predict future tumor growth that can be directly tested against experimental data. Acknowledgments: NCI P30CA068485, NCI R01CA138599, and NCI R25CA136440. References: 1. Atuegwu, et al. PMB, 2010. 2. Atuegwu, et al. MRM, 2011. 3. Atuegwu, et al. PMB, 2012. 4. Swanson, et al. Cell Prolif, 2000. 5. Jbaldi, et al. MRM, 2005. 6. Rockne, et al. PMB, 2010. 7. Ellingson, et al. MRM, 2011. 8. Christen, et al. NMR, 2010.

\section{8}

Real-time monitoring of apoptosis by natural killer cells using a caspase 3 sensor in human glioma cancer model. S.Y. Jeong ${ }^{1}$, H.W. Lee ${ }^{1}$, Y.H. Jeon $^{1}$, D. Singh ${ }^{1}$, S. Lee ${ }^{1}$, A. Rehemtulla ${ }^{2}$, B. Ahn ${ }^{1}$, J. Lee ${ }^{1}$; Kyungpook National University School of Medicine, Daegu, Korea (Rep.), ${ }^{2}$ University of Michigan, Ann Arbor, MI

Objective: The aim of this study is to real-time monitor apoptosis events of cancer cells by natural killer (NK) cells in a human glioma cancer model with a split luciferase-based caspase 3 sensor. Methods and Materials: Human glioma cancer (D54-MG) expressing a caspase 3 sensor was established (referred to as D54-C3S). NK92MI (IL-2 independent human natural killer cell line) cells were used as target cells. To monitor apoptosis events induced by NK92MI cells with caspase 3 sensor according to different ratios of target to effector $(1: 2.5,1: 5$, and 1:10), time course $(2,4$, 6 , and $8 \mathrm{~h}$ ), and effector stimulation with PMA/ionomycin, NK92MI cells were co-incubated with caspase 3 sensor and then bioluminescence signals were measured. Inhibition study using Z-VAD (a pan-caspase inhibitor) was performed to examine the selectivity of caspase 3 sensor. For in vivo study, five million NK92MI cells were intravenously administered to mice with D54-C3S tumor and bioluminescence was acquired at $6 \mathrm{~h}$ post-injection. Results: Bioluminescence signals from D54-C3S cells were increased in an effector number dependent manner and bioluminescence and effector/target ratio were well correlated $(1: 2.5,1: 5$, and $1: 10,7.8 \pm 0.5,10.7 \pm 0.6$, and $15.1 \pm 1.5$ fold, compared with control, $\mathrm{r} 2=0.97$ ). NK92MI cell treatment resulted in time-dependent increase of bioluminescence signals by activating caspase 3 sensor. Stimulated NK92MI cells revealed higher bioluminescence 
signals by caspase 3 sensor than non-stimulated NK92MI cells (at $5 \mathrm{~h}$, $12.5 \pm 0.3$ and $18.2 \pm 0.9$, non-stimulated NK92MI and stimulated NK92MI). Bioluminescence activity detected on cancer cells by NK92MI treatment was blocked by the use of a known pan-caspase inhibitor (Z-VAD). In vivo treatment of D54-C3S tumor with NK92MI cells resulted in $6 \sim 12$ fold induction of bioluminescence activity at $6 \mathrm{~h}$ after intravenous injection of the cells. Conclusions: We have succeeded in real-time monitoring of apoptosis mediated by NK cells in cancer cells both in vitro and in vivo. Bioluminescence imaging system with caspase 3 sensor system could be used as a useful tool for the optimization of NK-mediated immunotherapy.

\section{9}

Quantum dot-based molecular imaging for the detection of inflammation and cancer in mice. S. Kalangi ${ }^{1}$, N.K. Swarnakar ${ }^{2}$, R. Sathyavathi ${ }^{3}$, S. Jain ${ }^{2}$, D.N. Rao ${ }^{3}$, R. Pallu ${ }^{1}$; Department of Animal Sciences, University of Hyderabad, Hyderabad, India, ${ }^{2}$ National Institute of Pharmaceutical Education and Research (NIPER), S.A.S. Nagar, Punjab, India, ${ }^{3}$ School of Physics, University of Hyderabad, Hyderabad, India

One of the major hurdles in cancer treatment is the lack of a specific diagnosis in early stages. As inflammation is always associated with cancer, molecular imaging of the inflammatory lesions at early stages of cancer is one of the better options. To avoid the accumulation of nonspecific probe and to increase the accuracy of diagnosis we developed a novel quantum dot-based molecular imaging method which will address the situation. Tracing or imaging a single molecule in biological samples is a difficult objective and when the choice of a single molecule is a drug molecule, it is more complicated as it may lose its selectivity or activity towards the target after conjugation to a tracer or probe. In order to explore possibilities of covalent conjugation of a drug, it is important to select a functional group that will not result in the loss of function of the drug. Though in the recent past a few drug molecules have been conjugated to fluorophores, modifying the drug is not possible in all cases, as it is quite a complicated process and has its own disadvantages, like photobleaching. These disadvantages could be overcome by using the fluorescent nano particles, quantum dots (QDs). Semiconductor quantum dots have unique optical properties, such as longer fluorescence lifetime, tunable size, higher photobleaching thresholds and capacity for multiplex imaging, that outshine the conventional dyes. In the last decade, surface engineering and bio-functionalization techniques have transformed QDs into complex cellular probes capable of interaction with biomolecules. By monitoring its fluorescence signal, QDs enable us to understand basic properties, such as specific targeting and release rate of a drug molecule, within living systems. Owing to a number of advantages of aqueous phase synthesis, CdTe QDs have been the obvious choice for biological applications. In this work we have conjugated QDs to celecoxib, a potential specific inhibitor of a crucial enzyme, cyclooxygenase-2 (COX-2), which will be over expressed in cancer and inflammatory cells but not in normal cells. CdTe QDs were prepared using the reaction between $\mathrm{Cd}^{2+}$ and $\mathrm{NaHTe}$ solution in the presence of 3-mercaptopropionic acid (MPA) as a stabilizer; synthesized water soluble CdTe MPA capped QDs were checked for their fluorescence emission by fluorescence spectra; and high resolution transmission electron microscopy (HRTEM) was done to find out the size of QDs. To make sure they are not aggregated in solution, zeta potential was measured. These characterized QDs with $-\mathrm{COOH}$ functional group due to MPA were conjugated with $-\mathrm{NH}_{2}$ group of anti-inflammatory drug celecoxib with the help of a linker, 1-ethyl-3-[3-imethylaminopropyl] carbodiimide hydrochloride (EDC), by an optimized protocol at room temperature. QD-celecoxib conjugates were separated from un-reacted materials by ultracentrifugation and characterized for their fluorescence emission by fluorescence spectra and modified functional groups by FTIR analysis, and purity and structure predictions were solved with the analysis of $\mathrm{C}^{13}$ solid-state NMR. The QD-celecoxib conjugate's stability in aqueous solution (PBS) was checked by the zeta potential values. The characterized drug conjugates were tested for their specificity and efficacy to reach the inflammatory tissue by injecting them intravenously in a well-established carrageenan induced mouse paw edema model. Carrageenan $(50 \mu 1$ of $1 \%$ in sterile saline) was injected in the rear left footpad of mice, followed by (100 $\mu \mathrm{M}$ celecoxib conjugated) $5 \mathrm{mg} / \mathrm{kg}$ body weight of QD-celecoxib conjugates and unconjugated QDs injected into control and test groups of mice $(n=3)$ through the tail vein at 12 hours postcarrageenan. Animals were imaged 6 hours later with a two-photon in vivo imager with excitation at $475 \mathrm{~nm}$. Synthesized CdTe MPA capped QDs were orange red in color, excited at $475 \mathrm{~nm}$ and showed good emission at $529 \mathrm{~nm}$, as expected. HRTEM revealed that synthesized QDs have a size of $\sim 4 \mathrm{~nm}$. QD-celecoxib conjugates (10 nM to $100 \mu \mathrm{M}$ celecoxib) showed significant increases in comparison to control QDs, indicating that conjugation is a concentrationdependent process of the drug. Significant peaks at $1640 \mathrm{~cm}^{-1}(\mathrm{C}=\mathrm{O})$ and $1540 \mathrm{~cm}^{-1}$ (NH bending) in FTIR spectra of QD-drug conjugates gave clues about the formation of the amide bond between the - $\mathrm{COOH}$ group of MPA on QDs and the $-\mathrm{NH}_{2}$ group; the same was confirmed in $\mathrm{C}^{13}$ solid-state NMR of these conjugates with comparison to unconjugated QDs. QD-drug conjugates showed good stability, with zeta potential of 19 to $21 \mathrm{mV}$, which also confirms that there is no aggregation induced with drug conjugation. In vivo image of mice with inflamed paw shows the clear homogeneous distribution of unconjugated QDs throughout the body with no clear localization in any other parts of the body, including the inflamed paw. The mouse group which received the QD-celecoxib conjugates showed a very significant amount of localization of QD-drug conjugates, which demonstrates the clear imaging of COX-2 in inflammatory tissue. When the same animals were imaged at $12 \mathrm{hrs}$, the excretion of QDs through urinary bladder was observed and the intensity of fluorescence was greatly decreased throughout the body, except near the inflamed paw, but increased fluorescence was observed elsewhere in the mouse body. With the obtained results it is understood that molecular imaging with QDs by tagging a drug molecule is one of the better options to diagnose inflammation and cancer. Owing to the advantages of QDs - tunable size and specificity to react with drugs - bio-functionalization options (with various capping agents to provide the range of ligands) to match with functional groups on drugs of choice make them more efficient molecular probes. The same method can be used in the future for specific drug distribution, pharmacokinetic studies and to find its targets and nontarget interactions in mice at the laboratory level. As they can be used at minimal doses and are readily excreted post 6-8 hours, they are less toxic. Moreover, their high photobleaching threshold compared to the conventional chemical fluorophores enables them as suitable probes for imaging biological systems. However, detailed studies on this system may circumvent the radioisotope usage in this purpose. (Similar studies have been performed with a few other anti-inflammatory drugs [data not included in this abstract].)

\section{0}

Measuring and reducing the bias and variance of quantitative PET/CT imaging in multicenter clinical trials. D. Byrd ${ }^{1}$, R. Doot ${ }^{1}$, L. Dunnwald ${ }^{1}$, M. Muzi ${ }^{1}$, E. Schubert ${ }^{1}$, E. Shields ${ }^{1}$, T. Thompson ${ }^{2}$, B. Greer ${ }^{1}$, B. Kurland ${ }^{3}$, D. Mankoff ${ }^{4}$, H. Linden ${ }^{1}$, P. Kinahan ${ }^{1} ;{ }^{1}$ University of Washington, Seattle, WA, ${ }^{2}$ Seattle Cancer Care Alliance, Seattle, WA, ${ }^{3}$ Fred Hutchison Cancer Research Center, Seattle, WA, ${ }^{4}$ University of Pennsylvania, Philadelphia, PA

The pace of discovery of clinically useful cancer therapies can be accelerated by improved assessment of response. Quantitative molecular imaging can measure clinical efficacy and molecular mechanisms of agents. However, despite the general availability of positron emission tomography (PET) imaging using the glucose analogue $\left({ }^{18} \mathrm{~F}\right)$-fluorodeoxyglucose (FDG), FDG PET and PET/CT have not been widely incorporated into cancer clinical trials. A key factor impeding progress in this effort is inconsistency in quantitative measures from different sites, likely due to variability in imaging protocols and variable, non-optimal methods for image analysis. We present initial results from the development of local network tools to measure and reduce the bias and variance of multi-center quantitative PET/CT imaging measurements. Methods: Working with a regional network of community-based cancer treatment centers, we developed a set of imaging tools that monitor and track protocols and PET/CT scanner calibration of the local imaging sites. The scanner calibration tests use calibration source 'kits' with three components: (1) A NIST-traceable simulated F-18 source to evaluate and calibrate syringe-based dose calibrators. (2) An implicitly NIST-traceable $4.5 \mathrm{~cm}$ cylindrical source for PET scanner calibration. (3) An implicitly NIST-traceable source for well-counter calibration. Data are collected quarterly with these sources, using the local site's imaging protocol, and analyzed using robust analysis algorithms, which allow estimation of measurement error in the maximum standardized uptake value (SUVmax) due to instrumentation-related factors. In addition we track 
adherence to imaging protocols. The quantitative accuracy of performance of these protocols is monitored using test-retest patient imaging studies. The SUVs of known lesions are compared between two imaging sessions, which are typically less than 7 days apart in patients without treatment change Results are then compared with the variability of instrumentation-related factors and protocol adherence. Feedback to the community-based cancer imaging centers is also provided. Results: The accuracy of PET/CT images were widely varied due to instrumentation-related factors. The SUVmax bias ranged from $4 \%$ to $9 \%$ and was not stable in time. In addition positional alignment errors between PET and CT images were noted. We also found a variable degree of protocol adherence, most notably in the bio-distribution period between FDG injection and the start of the PET scan. While the cumulative impact of these factors can be difficult to estimate, our initial test-retest results ( $\mathrm{n}=3$ patients, 40 lesions) show that for the well-calibrated sites with strict protocol adherence the test-retest SUVmax was unbiased, with nearly all repeat measurements within $20 \%$ and 0.8 SUV units. Conclusions: Initial results indicate that inclusion of community-based $\mathrm{PET} / \mathrm{CT}$ imaging centers in clinical trials is feasible. However, monitoring of instrumentation-related and protocol adherence factors that affect SUV accuracy is needed to minimize measurement error and therefore optimize statistical power. The same factors will also impact the use of PET/CT imaging for patient-specific assessment of response to therapy Acknowledgments: Supported by NCI Contract No. 24XS036-004 and NIH grant U01-CA148131, a member of the Quantitative Imaging Network

\section{1}

${ }^{99} \mathrm{~m}$ Tc-etarfolatide (EC20) SPECT imaging for the identification of ovarian and non-small cell lung cancer patients who are most likely to benefit from folate-receptor-targeted agent vintafolide (EC145). P. $\mathrm{Kuo}^{1}$, R.W. Naumann ${ }^{2}$, J. Symanowski², B. Nguyen ${ }^{3}$, W. Harb ${ }^{4}$, M. Edelman ${ }^{5}$; ${ }^{1}$ University of Arizona, Tucson, AZ, ${ }^{2}$ Levine Cancer Institute, Charlotte, NC, ${ }^{3}$ Endocyte Inc., Indianapolis, IN, ${ }^{4}$ Horizon Oncology Research, Inc., Lafayette, IN, ${ }^{5}$ Greenebaum Cancer Center, Baltimore, MD

Background: ${ }^{99 \mathrm{~m}} \mathrm{Tc}$-etarfolatide is a conjugate of folic acid (FA) and a tripeptide moiety that efficiently chelates technetium $99 \mathrm{~m}$. It allows for fullbody, real-time single-photon emission computed tomography (SPECT) imaging of folate receptor (FR) expression of individual tumors in patients. Vintafolide is a novel drug conjugate of FA and the vinca alkaloid desacetylvinblastine hydrazide (DAVLBH). Herein, we describe the use of ${ }^{99 \mathrm{~m} T c}$-etarfolatide imaging to identify FR-expressing tumors and correlate FR expression with benefit from vintafolide. Methods: Two single-arm phase II studies were conducted in heavily pre-treated patients with ovarian and non-small cell lung cancer (OC, NSCLC). Patients in both studies were imaged with ${ }^{99 m}$ Tc-etarfolatide SPECT scans to assess for uptake in the target lesions (selected per RECIST criteria) and then treated with singleagent vintafolide. Patients were categorized as FR $(100 \%)$ (all target lesions FR positive), FR (10-90\%) (at least 1 but not all target lesions FR positive), or FR $(0 \%)$ (no FR-positive target lesions). Vintafolide was administered in an induction phase (1.0 mg intravenous [IV] bolus daily x 5 days for 3 weeks of a 4-week cycle $\mathrm{x} 2$ cycles) followed by a maintenance phase ( $2.5 \mathrm{mg}$ IV bolus daily x 3 days, weeks 1 and 3 of a 4 -week cycle). Patients received therapy until disease progression or unacceptable toxicity. Results: The greatest efficacy was observed in FR $(100 \%)$ patients. These patients experienced at least a doubling of progression-free survival (PFS) compared with FR (10-90\%) patients (OC, 15.2 vs 7.4 weeks; NSCLC, 31.1 vs 7.3 weeks). The PFS hazard ratios (HR) with $95 \%$ confidence interval (CI) between the 2 FR subgroups were $0.797(0.362,1.756)$ and $0.326(0.115$, $0.919)$ for the OC and NSCLC studies, respectively. The FR (100\%) subgroup also had $>50 \%$ improvement in disease control rate (OC, $57.1 \%$ vs $36.4 \%$; NSCLC, $57.1 \%$ vs $14.3 \%$ ) and overall survival (OS) (OC, 63.6 vs 41.7 weeks; NSCLC, 47.2 vs 14.9 weeks) compared with FR (10-90\%) patients. The OS HR $(95 \% \mathrm{CI})$ between the 2 FR subgroups were 0.574 $(0.213,1.542)$ and $0.539(0.209,1.395)$ for the OC and NSCLC studies, respectively. Furthermore, analyses of tumor lesions showed that the percentage of lesions that were decreased (at least $20 \%$ decrease in size from baseline) or stable $(<20 \%$ increase in size from baseline) was greater in FR-positive lesions than in FR-negative lesions, supporting the targetspecific mechanism of action of vintafolide. ${ }^{99 \mathrm{~m}} \mathrm{Tc}$-etarfolatide was safe and well tolerated, with an overall low drug-related adverse events rate. Conclusions: Data from studies in heavily pre-treated OC and NSCLC patients support this combined approach to personalized therapy using ${ }^{99 m}$ Tc-etarfolatide to identify patients most likely to benefit from FR-targeted treatment with vintafolide.

\section{2}

Serial whole-body quantification of metabolic tumor burden on ${ }^{18} \mathrm{~F}$-fluorocholine PET/CT in castrate-resistant prostate cancer. S.A. Kwee, A. Watanabe, M. Coel, J. Lim; Hamamatsu/Queen's PET Imaging Center, The Queen's Medical Center, Honolulu, HI

Purpose: Examine metabolically active tumor volume (MATV) and total lesion activity (TLA) (lesion mean standardized uptake value (SUV) $\mathrm{x}$ MATV) on ${ }^{18} \mathrm{~F}$-fluorocholine (FC) PET/CT as potential measures of wholebody tumor burden and metabolic treatment response in castrate-resistant metastatic prostate cancer (CRPC). Methods: Serial whole body ${ }^{18} \mathrm{~F}$-fluorcholine PET/CT scanning was performed in 22 patients before and 28-224 days following treatment with total androgen blockade or withdrawal. Activity thresholds were used to define volumetric regions of interest (VOIs) to measure tumor SUVmax, SUVpeak, MATV, and TLA. Estimates of whole-body tumor burden based on net MATV and TLA for each patient were compared with serum prostate specific antigen (PSA) levels measured over the course of treatment. Results: Median PSA at baseline was $15.0 \mathrm{ng} / \mathrm{mL}$ (range 2.14 to $81.0 \mathrm{ng} / \mathrm{mL}$ ). Tumor sites were localized by PET to the skeletal system in 9 patients, lymph nodes in 1 patient, and both skeletal and extra-skeletal sites in 12 patients. Net MATV at baseline ranged from $0.12 \mathrm{cc}$ to $1544 \mathrm{cc}$ (median $36.6 \mathrm{cc}$ ). Baseline net TLA ranged from $0.4 \mathrm{~g}$ to $6689 \mathrm{~g}$ (median $142.7 \mathrm{~g}$ ). The PSA level at baseline, but not PSA velocity or doubling time, correlated with net MATV and TLA from the baseline FC PET/CT exam (Spearman $\rho=0.0 .47$, $\mathrm{p}=0.026 ; \rho=0.49, \mathrm{p}=0.021$, respectively). Follow-up FC PET/CT was consistent with tumor progression in 8 cases. Change in net MATV ranged from $-416 \mathrm{cc}$ to $+1224 \mathrm{cc}$, while change in net TLA ranged from $-1689 \mathrm{~g}$ to $+6123 \mathrm{~g}$. PSA levels at follow-up were significantly correlated with both net MAV and net TLA on follow-up FC PET/CT $(\rho=0.82, p<0.0001$; $\rho=0.83, p<0.0001$, respectively) as well as percent change in net MATV and net TLA $(\rho=0.50, p=0.017 ; \rho=0.53, p=0.01$, respectively). Conclusion: Correlations between serum PSA and MATV/TLA preliminarily support FC PET/CT-based assessment of whole-body tumor burden in CRPC. The potential to anatomically localize tumor progression and response with FC $\mathrm{PET} / \mathrm{CT}$ may prove complementary to PSA-based characterization of treatment response in CRPC.

\section{3}

Luciferase imaging of ER $\alpha$-AIB1 interaction. M. Lake, Q. Nguyen, S. Ali, E. Aboagye; Imperial College, London, United Kingdom

Estrogen receptor alpha $(\mathrm{ER} \alpha)$ is a nuclear receptor transcription factor which has been implicated in the initiation and progression of breast cancer. Approximately two thirds of all breast cancers express ER $\alpha$ and the majority of these tumors respond to adjuvant anti-estrogen treatment. However, though current therapies have greatly enhanced patient prognosis, there continues to be an impetus for the development of improved anti-estrogens. We have previously described the development of a split-luciferase assay to image the association of ER $\alpha$ with its coactivator AIB1 as a novel means of gauging ER $\alpha$ activation. AIB1 is an ER $\alpha$ coactivator which is thought to be of particular importance for estrogen (E2) signaling in breast cancer. Fusion protein constructs, in which the ER $\alpha$ ligand binding domain (ER-LBD) and AIB1 nuclear receptor interaction domain (AIB1-RID) are fused to the Nand C-terminal fragments of firefly luciferase, were previously produced and validated for in vitro and in vivo studies by transient transfection of 293 cells. Here we describe the generation of an MCF7 cell line (M.EL/AR) which stably expresses the luciferase fragment fusion proteins. It is foreseen that this cell line could provide a valuable high-throughput tool for screening novel ER $\alpha$ targeted therapies in a cellular context in vitro and in vivo. Incubation of M.EL/AR cells with E2 produces a dose dependent increase in luciferase activity that can be modulated by the anti-estrogens tamoxifen and ICI 182,780. Time-course studies indicate that these ligand-induced alterations in the luciferase signal can be observed 2 hours after ligand addition. The potential of the assay for ascertaining the ER $\alpha$-specific activity of estrogen receptor targeted ligands has been demonstrated using 
the ER $\beta$-specific ligands DPN and ERB041. It is anticipated that the cell line will also be of great value for screening next-generation compounds which aim to interrupt $\mathrm{ER} \alpha$-coactivator interaction.

44

Luciferase imaging of H3K9 methylation. M. Lake, E. Aboagye; Imperial College, London, United Kingdom

Epigenetic modifications are responsible for the multitude of phenotypes which can be produced from a single genotype. These epigenetic modifications, which largely take the form of DNA methylation and posttranslational modification of histone proteins, influence nucleosome packing to produce a more 'open' or 'closed' chromatin structure, which promotes or inhibits gene expression. Deregulation of the epigenetic code is observed in many human cancers and numerous other diseases. Di- and trimethylated histone $\mathrm{H} 3$ lysine $9(\mathrm{H} 3 \mathrm{~K} 9 \mathrm{me} 2 / 3)$ is a marker of transcriptionally repressed chromatin, which can be associated with the inappropriate silencing of tumor suppressor genes. The enzymes responsible for this modification have been shown to be deregulated in a number of different cancers, and knock down of these enzymes can reduce the proliferative and invasive properties of cancer cells. In order to image $\mathrm{H} 3 \mathrm{~K} 9 \mathrm{me} 2 / 3$ we have developed an intramolecular HP $1 \beta$ split-luciferase construct. HP $1 \beta$ is a member of the heterochromatin protein 1 family, which includes HP $1 \alpha$ and $\mathrm{HP} 1 \gamma$. HP $1 \beta$ specifically binds to $\mathrm{H} 3 \mathrm{~K} 9 \mathrm{me} 2 / 3$ in heterochromatin and mediates gene silencing. We aim to non-invasively image $\mathrm{H} 3 \mathrm{~K} 9 \mathrm{me} 2 / 3$ by exploiting conformational changes associated with this HP1 $\beta$ binding; we have produced fusion proteins of HP1 $\beta$ flanked with two enzymatically inactive fragments of firefly luciferase, which can complement when brought into close proximity. Initial studies indicate that incubation with the pan-methyltransferase inhibitor Adox produces a time- and dose-dependent increase in luciferase activity which correlates with alterations in $\mathrm{H} 3 \mathrm{~K} 9$ methylation

\section{5}

Predicting response to vintafolide therapy using the folate-receptorspecific imaging agent, ${ }^{99 \mathrm{~m}}$ Tc-etarfolatide. C.P. Leamon, J.A. Reddy, P.J. Klein, I.R. Vlahov, M. Vetzel, A. Bloomfield, R. Dorton, M. Nelson; Endocyte Inc., West Lafayette, IN

Background: Vintafolide is a potent folate-targeted Vinca alkaloid conjugate that is currently being evaluated in a global phase 3 trial for the treatment of platinum-resistant ovarian cancer in combination with pegylated doxorubicin, as well as a randomized phase 2 study for advanced non-small-cell lung carcinoma. By design, response to vintafolide is dependent on folate receptor (FR) expression. Therefore, having the ability to screen patients for FR-positive disease in real time could predictably increase the efficiency of, and decrease the time for, clinical investigations of FR-targeted therapies. One promising method that is currently being co-developed along with vintafolide is the imaging of patients with ${ }^{99 \mathrm{~m}} \mathrm{Tc}$ etarfolatide, a small-molecular-weight folate-targeted companion imaging agent that can be used for the non-invasive, real-time assessment of functionally active as well as anatomically accessible FRs. Methods: The pharmacologic properties of vintafolide and ${ }^{99 \mathrm{~m}} \mathrm{Tc}$-etarfolatide were investigated in vitro and in vivo using a panel of human and murine cell lines, and established subcutaneous xenograft models. Vintafolide in vitro cytotoxicity was measured using a ${ }^{3} \mathrm{H}$-thymidine incorporation assay, and ${ }^{99 \mathrm{~m}} \mathrm{Tc}$-etarfolatide uptake quantified by standard gamma radiation detection. For in vivo assessments, both CT and SPECT imaging methodologies were applied to precisely identify the anatomical location of ${ }^{99 \mathrm{~m}} \mathrm{Tc}$-etarfolatide tissue uptake. Results: Similar to vintafolide, ${ }^{99 \mathrm{~m}} \mathrm{Tc}$-etarfolatide binds to the FR with high affinity ( $\mathrm{Kd} \sim 3 \mathrm{nM}$ ) in a saturable manner and independent of cell type. The binding specificity of ${ }^{99 \mathrm{~m}} \mathrm{Tc}$-etarfolatide has been confirmed using validated preclinical models where it was found that: (i) binding of ${ }^{99 \mathrm{~m} T c-e t a r f o l a t i d e ~ t o ~ c e l l s ~ o r ~ i t s ~ a c c u m u l a t i o n ~ w i t h i n ~ s o l i d ~}$ tumors is dependent on FR expression; and (ii) excess folic acid can competitively block ${ }^{99 \mathrm{~m}}$ Tc-etarfolatide's cell/tissue binding. Furthermore, vintafolide was also found to displace ${ }^{99 \mathrm{~m}} \mathrm{Tc}$-etarfolatide when pre-bound to FR-positive tumors in mice, strongly suggesting that vintafolide and ${ }^{99 \mathrm{~m}} \mathrm{Tc}-$ etarfolatide are distributed similarly in vivo. Conclusions: These findings indicate that the FR mediates tissue binding of ${ }^{99 \mathrm{~m}} \mathrm{Tc}$-etarfolatide, and that this radiodiagnostic agent may predict which tissues will accumulate and potentially respond to folate-containing drugs, such as vintafolide. This novel and personalized approach may allow for the identification of patients who would most likely benefit from FR-targeted therapy.

\section{6}

Imaging secreted proteases in metastatic prostate cancer using novel human antibodies. A.M. LeBeau, C.S. Craik, H.F. VanBrocklin; University of California, San Francisco, CA

New therapeutics for castration-resistant prostate (CRPC) are urgently needed. The development of such drugs is greatly hampered by the inability to measure therapeutic response in a majority of patients. New imaging agents are desperately needed to aid in the development of therapeutics for CRPC. One attractive target for imaging CRPC is the plasminogen activation system (PAS). Consisting of the secreted serine protease urokinase plasminogen activator (UPA), the receptor of uPA (UPAR) and the endogenous inhibitor of uPA (PAI-1), the PAS is highly over-expressed in metastatic CRPC. Using a human fragment of antigen binding phage display library, we have developed a novel human antibody that inhibits secreted and uPAR bound forms of uPA. The anti-uPA antibody, U33 IgG, was found to be a potent competitive inhibitor of uPA and was highly selective demonstrating no cross-reactivity with other secreted prostate cancer-associated proteases. Subsequent in vitro studies, using U33 IgG labeled with ${ }^{111} \mathrm{In}\left({ }^{111} \mathrm{In}-\mathrm{U} 33 \mathrm{IgG}\right)$, found that the U33 mimicked the function of PAI-1 resulting in internalization of the UPA-U33 complex when bound to uPAR. As a SPECT/CT imaging probe, ${ }^{111} \mathrm{In}-\mathrm{U} 33 \mathrm{IgG}$ demonstrated a high tumor uptake of $43 \% \mathrm{ID} / \mathrm{g}$ at $72 \mathrm{hrs}$ in PC3 xenograft mice. The localization and internalization of ${ }^{111} \mathrm{In}-\mathrm{U} 33 \mathrm{IgG}$ in vitro and in vivo was blocked by prior administration of excess cold U33 IgG. The ability of ${ }^{111}$ In-U33 IgG to image small, dispersed lesions was tested in a PC3 intracardiac dissemination model. The ${ }^{111} \mathrm{In}-\mathrm{U} 33 \mathrm{IgG}$ probe was able to detect both soft tissue and osseous lesions in this model. Excision and homogenization of the lesions found that they contained high levels of uPAmediated proteolysis that could be inhibited by U33 IgG. In summation, we have developed a novel and potent new PAS tracer that shows promise for imaging metastatic CRPC

\section{7}

${ }^{111}$ In-CSL360 mAb modified with nuclear localization signal containing peptides specific for the leukemic stem cell phenotype CD123+CD131for leukemia detection and Auger electron radioimmunotherapy. J.V. Leyton $^{1}$, B. Williams ${ }^{2}$, A. Keating ${ }^{2}$, J.E. Dick ${ }^{1}$, M. Minden ${ }^{2}$, R.M. Reilly ${ }^{1}$; ${ }^{1}$ University of Toronto, Toronto, ON, Canada, ${ }^{2}$ University Health Network, Toronto, ON, Canada

Objective: Unlike the rapidly dividing cells that are a major characteristic of acute myelogenous leukemia (AML), it has been postulated that one reason for therapy failure with conventional chemotherapy is the inability to eradicate the leukemic stem cell (LSC). In addition there is a need for new technologies that target specific biomarkers in order to more accurately monitor AML. LSCs have been shown to be predictive of poor survival outcome in patients with AML; therefore a biomarker discriminating against LSCs would be an attractive target. The functional interleukin-3 receptor (IL-3R) is composed of the alpha-subunit (CD123) and the betasubunit (CD131). CD123 is overexpressed on leukemic blasts and LSCs in the absence of CD131. The chimeric mAb CSL360 recognizes an epitope on CD123 exposed in the absence of CD131 on LSCs but not on normal hematopoietic stem cells. Thus, our aim was to construct a radioimmunoconjugate capable of therapy through eradication of leukemic blasts and LSCs while simultaneously avoiding healthy bone marrow (BM) cells expressing the functional IL-3R by radiolabeling CSL360 mAb with ${ }^{111}$ In and modified with nuclear localization sequence (NLS) containing peptides for nanometer-micrometer Auger electron radiotherapy. In addition, we utilize the 171 and $245 \mathrm{keV}$ gamma photons for high-resolution micro-single photon emission computerized tomography (microSPECT) imaging of leukemic sites in NOD/SCID mice. Methods: MAb CSL360 was directly derivatized with DTPA then reacted with sulfo-SMCC to introduce maleimide for conjugation to NLS containing peptides 
(CGYGPKKKRKVGG) and subsequently complexed with ${ }^{111}$ In $\left({ }^{111}\right.$ In-NLS-CSL360). First, ${ }^{111}$ In-NLS-CSL360 was evaluated for the ability to detect and decrease the CD123+ AML population in the femurs of primary AML engrafted NOD/SCID mice. At 8-weeks post-engraftment, mice were injected with either $451 \pm 35.2 \mu \mathrm{Ci} /$ mouse of ${ }^{111}$ In-NLS-CSL360 or $495 \pm 21.1^{111}$ In-NLS-isotype control (IgG). Targeting was evaluated at $72 \mathrm{~h}$ post injection (pi) by microSPECT/CT followed by biodistribution and harvesting of BM cells and evaluation of the CD45+CD123+ population by flow cytometry. Second, a long-term therapy was evaluated. NOD/SCID mice were inoculated with primary AML cells and subsequently administered $122 \pm 4.2,132 \pm 1.7$, or $119 \pm 1.6 \mu \mathrm{Ci} /$ mouse of ${ }^{111}$ In-NLSCSL360, ${ }^{111}$ In-CSL360, or ${ }^{111}$ In-NLS-IgG 96 h later, respectively. 8 weeks after therapy mice were sacrificed, AML burden in the BM was assessed by flow cytometric analysis. Complete blood counts $(\mathrm{CBC})$ were performed on whole blood to evaluate hematological parameters. 1-way ANOVA with Dunnett's multiple comparisons test were performed. Results: ${ }^{111}$ In-NLSCSL360 demonstrated the ability to provide high contrast imaging of AML in the BM of NOD/SCID mice and high specific uptake compared to ${ }^{111}$ In-NLS-IgG into the femurs $(8.8 \pm 1.4$ vs $3.0 \pm 0.1 \% \mathrm{ID} / \mathrm{g} ; \mathrm{p}<0.05)$ of AML engrafted NOD/SCID mice at $72 \mathrm{~h}$ pi. CD45+CD123+ cells were significantly reduced with ${ }^{111}$ In-NLS-CSL360 compared to ${ }^{111}$ In-NLS-IgG $(47.2 \pm 7.7 \%$ vs $82.3 \pm 1.8 \%, p=7.92 \mathrm{E}-06) .{ }^{111}$ In-NLS-CSL360 reduced the leukemic burden (CD45+ cells) to $64.6 \pm 32.6 \%$ compared to untreated ( 88.8 $\pm 5.4 \% ; \mathrm{p}<0.05),{ }^{111} \mathrm{In}-\mathrm{CSL} 360(84.5 \pm 1.6 \%)$, and ${ }^{111} \operatorname{In}-\mathrm{NLS}-\operatorname{IgG}(84.5 \pm$ $5.5 \%)$ treated mice, respectively. The LSC population $(\mathrm{CD} 34+\mathrm{CD} 38-$ $\mathrm{CD} 123+)$ also was reduced $13.4 \pm 6.1 \%$ compared to untreated $(25.0 \pm$ $6.3 \% ; \mathrm{p}<0.01),{ }^{111}$ In-CSL360 (20.5 $\left.\pm 3.0 \%\right)$, and ${ }^{111}$ In-NLS-IgG $(21.4 \pm$ $1.8 \% ; \mathrm{p}<0.05)$ treated mice, respectively. $\mathrm{CBC}$ analyses determined that ${ }^{111}$ In-NLS-CSL360 could significantly reduce the leukemic cell population and maintain healthy hemoglobin levels in the blood compared to untreated NOD/SCID mice. Conclusion: ${ }^{111}$ In-NLS-CSL360 can localize to and detect CD123+ leukemic cells in the BM of AML engrafted NOD/SCID mice. Ultra-short range Auger electrons from ${ }^{111}$ In can be deposited inside the nucleus of LSCs in vivo by ${ }^{111}$ In-NLS-CSL360 producing a greater antileukemic effect by eradication of the LSC population. Acknowledgment: Supported by the Canadian Institutes of Health Research.

\section{8}

Kinetics and allometry models for radiopharmaceutical dosimetry using LUTATE. M.F. Lima, N.G. Silva, C.H. de Mesquita; Nuclear Research Institute-IPEN, São Paulo, Brazil

One model based in compartmental analysis using gamma camera imaging was employed to determine the kinetic constants of LUTATE to three animal species (Wistar rat, Armenian hamster and Syrian hamster) in biodistribution studies. Every one of the biodistribution curves were split in two phases: phase 1, governed by uptake from the blood to the organs and tissues, and phase 2 , governed by the renal excretion. The kinetic constants obtained from the animals' data were used to build allometric scales to predict radiopharmaceutical biodistribution in humans, employing relations by body mass, by metabolism, by life span and by physiological parameters. The allometric relations from kinetic data to the organs responsible for LUTATE metabolism and excretion - liver, kidneys and urinary bladdershow good correlation in the extrapolation by body mass and metabolism $\left(\mathrm{R}^{2}>0.70\right)$ and by physiological parameters $\left(\mathrm{R}^{2}=1\right)$ to phase 1 of the biodistribution studies. To phase 2 , only the kinetic data from the blood to the liver and from the blood to the kidneys show good correlation $\left(\mathrm{R}^{2}>0.83\right)$. These extrapolation results were compared with PRRT (peptide receptor radiotherapy) patients' kinetic data calculated using one model developed for humans. The kinetic constants obtained from humans were used in dose assessment for PRRT patients, considering 26 organs and tissues described in the MIRD methodology. The dosimetry results, based on the kinetic constants and calculated by compartmental analysis, were in agreement with the few available results from literature and allow dose estimation in other sensitive tissues that are often difficult to discriminate in PET/CT or SPECT/CT studies.

\section{9}

Effect of bis(acetylacetonato)oxovanadium(IV) on uptake of 2-(fluorine18)-2-deoxyglucose in positron emission tomography imaging. M.W.
Makinen, R. Bamba, L. Ikejimba, C. Wietholt, S.D. Conzen, C. Chen; The University of Chicago, Chicago, IL

In clinical settings, cancer detection by positron emission tomography (PET) imaging with 2-(fluorine-18)-2-deoxy-D-glucose (FDG) ordinarily requires tumors of dimension $\geq 10 \mathrm{~mm}$. Increasing the fractional uptake of FDG by cancer tissue would provide a means to detect smaller neoplasms. We have investigated whether bis(acetylacetonato)oxovanadium(IV) $\left[\mathrm{VO}(\mathrm{acac})_{2}\right]$ can facilitate enhanced uptake of FDG by cultured MDAMB-231 breast carcinoma cells. After overnight growth in Dulbecco's modification of Eagle Medium containing $25 \mathrm{mM}$ D-glucose, $10 \%$ fetal calf serum, penicillin $(100 \mathrm{U} / \mathrm{mL})$, and streptomycin $(100 \mathrm{U} / \mathrm{mL})$ in 12-well plates at $37{ }^{\circ} \mathrm{C}$, cells were washed, and the medium was replaced with Krebs-Ringer solution buffered with HEPES (KRBH) containing $0.1 \mathrm{mM}$ BSA and $5 \mathrm{mM}$ glucose. 1-25 $\mu \mathrm{M} \mathrm{VO(acac})_{2}$ was added for $2 \mathrm{hr}$ incubation of cells followed by addition of FDG $(c a .0 .01 \mathrm{mCi} /$ well) with incubation further for $1 \mathrm{hr}$. FDG uptake was quenched by placing the 12-well plate on ice and washing the cells with cold $\mathrm{KRBH}$. Cells were imaged with a GMI FLEX Triumph tri-modal imaging system, and data were analyzed with use of AMIRA (Visage Imaging, San Diego, CA) to estimate the fractional population of voxel intensities in each region of interest. An approximate linear increase in the fractional population of voxels was observed with intensity greater than the mean value of control cells over a $1-12 \mu \mathrm{M}$ $\mathrm{VO}(\mathrm{acac})_{2}$ range, and on average $89 \%$ of $\mathrm{VO}(\mathrm{acac})_{2}$-treated cells had greater intensity than the mean value of control cells. There was no further increased capacity of cells for enhanced fractional uptake of FDG over the 12-25 $\mu \mathrm{M}$ $\mathrm{VO}(\mathrm{acac})_{2}$ range. In preliminary studies using xenograft tumors generated with MDA-MB-231 cells in female SCID mice, we have similarly observed enhanced FDG uptake by tumor tissue. Investigations are being conducted to determine the minimal $\mathrm{VO}(\mathrm{acac})_{2}$ dosage and optimal time interval between injection of $\mathrm{VO}(\mathrm{acac})_{2}$ and onset of FDG PET imaging. Acknowledgments: Research supported by NIH (P50 CA125183, P30 CA14599, and S10 RR022520).

\section{0}

A simplified approach for ${ }^{18} \mathrm{~F}$ radiolabeling of biomarker-binding peptides for PET imaging of cancer as part of a theranostic approach. W.J. McBride ${ }^{1}$, R.M. Sharkey ${ }^{1}$, P. Laverman ${ }^{2}$, R. Schoffelen ${ }^{2}$, O.C. Boerman ${ }^{2}$, D.M. Goldenberg ${ }^{3} ;{ }^{1}$ Immunomedics, Inc., Morris Plains, NJ, ${ }^{2}$ Radboud University, Nijmegen, The Netherlands, ${ }^{3}$ Garden State Cancer Center, Morris Plains, NJ

Objective: ${ }^{18} \mathrm{~F}$-FDG has contributed to a paradigm change in cancer management by providing a functional imaging method for assessing proliferation, particularly in staging and monitoring cancer patients. To extend molecular cancer imaging to cancer-targeting peptides, we have developed a rapid and simple, broadly applicable, procedure for radiolabeling tumor biomarker imaging peptides with ${ }^{18} \mathrm{~F}$ in high yield and/ or at high specific activity. The specific images should provide a basis for assessing targeted therapeutic that is designed to treat a specific cancer optimally while minimizing toxicity to normal tissues, thus providing a theranostic approach for personalized cancer therapy. Methods: Lyophilized kits containing the candidate peptide that is modified with a metal-binding ligand have been formulated in the presence of aluminum. The labeling kits greatly reduce the complexity and the time needed to label the peptides with ${ }^{18} \mathrm{~F}$, the preferred isotope for PET imaging. The peptides are radiofluorinated simply by adding ${ }^{18} \mathrm{~F}^{-}$in saline with a small quantity of ethanol, and after a brief heating, they are quickly purified using solid-phase extraction (SPE) cartridges. For proof-of-concept, we prepared lyophilized kits containing a hapten-peptide (IMP485) that is used in a bispecific antibody pretargeting procedure for highly sensitive and specific targeting of tumor antigens based on the specificity of a bispecific antibody (pretargeting solid tumors, such as colorectal, pancreatic, lung, prostate, breast and ovarian), as well as a somatostatin-binding peptide, octreotide (neuroendocrine tumors). The kits were optimized by examining the effects of $\mathrm{pH}$, reaction time, temperature, peptide concentration, different buffers, $\mathrm{Al}^{3+}$ peptide ratios, the addition of a co-solvent, and an antioxidant. Results: An optimized kit containing $20 \mathrm{nmol}$ of IMP485 was labeled with ${ }^{18} \mathrm{~F}$ in a single step, with a specific activity up to $222 \mathrm{GBq} / \mu \mathrm{mol}(6015 \mathrm{Ci} / \mathrm{mmol})$ in high isolated yield (45-97\%) after a simple SPE purification. The total reaction and purification time was about 20 min. Optimal IMP485-kit labeling yields 
were observed at $\mathrm{pH} 4.0$, stabilized by the nonvolatile buffer, potassium biphthalate. Nearly quantitative radiolabeling yields were obtained with as little as $40 \mathrm{nmol}$ of IMP485. Slightly lower labeling yields were obtained with the NOTA ligand on octreotide, but high specific activities were still achievable and good yields were obtained even at $50^{\circ} \mathrm{C} .{ }^{18} \mathrm{~F}$-IMP485 was cleared rapidly in mice, with minimal tissue retention and in a pretargeting setting, excellent tumor/nontumor ratios were achieved in just $1 \mathrm{~h}$ Conclusions: Optimized, facile, lyophilized kits containing peptides conjugated with NOTA/NODA were developed and found to be generally useful for preparing ${ }^{18} \mathrm{~F}$-peptides quickly and efficiently. This simplified approach potentially could be applied to a wide range of peptides for imaging cancer.

\section{1}

Aluminum fluoride [F-18] radiolabeling of proteins for same-day imaging of tumors by positron-emission tomography. W.J. McBride ${ }^{1}$, R.M. Sharkey ${ }^{1}$, D.M. Goldenberg ${ }^{2}$; ${ }^{1}$ Immunomedics, Inc., Morris Plains, $\mathrm{NJ},{ }^{2}$ Garden State Cancer Center, Morris Plains, NJ

Objectives: Antibody-derived agents are highly versatile and specific for targeting cancer and other diseases. While the pharmacokinetic properties of some of these agents are often too long for use with ${ }^{18} \mathrm{~F}$, others with more rapid clearance could be adapted for use with ${ }^{18} \mathrm{~F}$, the most commonly used and ideally suited radionuclide for PET imaging. We developed a rapid method for binding ${ }^{18} \mathrm{~F}$ to peptides, using a metal-capturing chelate to bind tightly formed aluminum-fluoride complexes. Herein, we investigated ways to adapt this rapid procedure for preparing heat-labile ${ }^{18} \mathrm{~F}$-labeled compounds, such as antibody-derived fragments, in good yields and in just 45 minutes. Methods: A 2-(4-(carboxymethyl)-7-\{[4-(carboxymethyl) phenyl]methyl\}-1,4,7-triazacyclononan-1-yl)acetic acid (NODA-MPAA) aluminum fluoride binding-ligand was synthesized, and attached to 2-aminoethyl-maleimide to form NODA-MPAEM (MPAEM = methyl phenyl acetamido ethyl maleimide) for conjugation to thiols on proteins. The maleimide was radiolabeled in one step by adding ${ }^{18} \mathrm{~F}^{-}$in saline to the NODA-MPAEM solution and an $\mathrm{Al}^{3+}$ solution heated at $100^{\circ} \mathrm{C} / 15 \mathrm{~min}$. This product was used as is or purified rapidly by solid phase extraction (SPE) and added to either an anti-CEACAM5 Fab' (2.4 thiols/Fab') or an antiCEACAM5 diabody, which were formulated at $1 \mathrm{mg}(20 \mathrm{nmol}) / \mathrm{vial}$ with an acetate buffer and a bulking agent and then lyophilized. After a $10 \mathrm{~min}$ incubation, the product was purified on a Sephadex column to obtain the $\mathrm{A} 1^{18} \mathrm{~F}$-labeled proteins. Results: The NODA-MPAEM intermediate was labeled with $37-1,481 \mathrm{MBq}$ F-18 with $\mathrm{Al}{ }^{18} \mathrm{~F}-\mathrm{NODA}-\mathrm{MPAEM}$ yields ranging from $49-93 \%$ yield depending on the reaction conditions used. The SPE-purified $\mathrm{Al}^{18} \mathrm{~F}$-NODA-MPAEM was added to the anti-CEACAM5 $\mathrm{Fab}^{\prime}$, which was isolated in $\sim 80 \%$ yield in a two-step process in $\sim 45 \mathrm{~min}$. The diabody was labeled in a similar manner as the Fab' but the labeled $\mathrm{Al}^{18} \mathrm{~F}$-maleimide intermediate was not purified before addition to the diabody. Using this process the AlF-18-diabody was obtained in $37 \%$ isolated yield. Both products retained immunoreactivity. Conclusions: The NODA-MPAEM ligand was synthesized to label temperature-sensitive molecules such as proteins using the $\mathrm{Al}{ }^{18} \mathrm{~F}$ method. We show that antibody fragments (Fab' and diabody) can be prepared in good yields (40-80\%) in 45 min without losing immunoreactivity. This procedure would allow rapid preparation for same-day PET imaging with antibody-derived targeting agents.

\section{2}

Translocator protein (TSPO) as an imaging target in pancreatic cancer. E.T. McKinley, D. Tang, N. Merchant, C. Shi, H.L. Moses, H.C. Manning; Vanderbilt University, Nashville, TN

Despite improvements in imaging techniques, surgery and oncologic treatments, clinical outcome for pancreatic cancer has not improved over the past 25 years. In order to modify these statistics, it is imperative to develop new imaging capabilities that will allow for earlier detection of lesions at the time when the disease is still at a curable stage. Molecular imaging biomarkers to detect and characterize pancreatic cancer remain a critical unmet clinical need. Only one positron emission tomography (PET) tracer is routinely used in this setting $\left({ }^{18} \mathrm{~F}-\mathrm{FDG}\right)$ and its sensitivity and specificity are severely limited. We have identified translocator protein (TSPO), an $18 \mathrm{kDa}$ outer mitochondrial membrane protein involved in a number of cellular processes including steroid synthesis, cholesterol metabolism, proliferation, and apoptosis as a potential biomarker of early pancreatic neoplasias. Elevated levels of TSPO are well-documented in oncology (e.g., colon, breast, glioma, oral), where levels correlate with tumor proliferation, invasion, and metastasis. In tumor microarrays (TMAs) of human pancreatic cancer, we observed significantly elevated TSPO levels in a spectrum of neoplastic pancreas lesions ranging from precursor pancreatic intraepithelial neoplasias (PanINs) lesions to advanced pancreatic adenocarcinoma compared to normal pancreas and chronic pancreatitis. Novel TSPO PET ligands developed in our laboratory have proven highly cancer-specific in preclinical studies of glioma and colon cancer. As an extension of these studies, we recently evaluated the TSPO ligand ${ }^{18} \mathrm{~F}$-VUIIS-1018 in a genetically engineered mouse model of pancreatic cancer. Supporting TSPO PET as a metric to detect primary pancreatic lesions, we observed robust tracer uptake in pancreatic tumors arising in $\mathrm{Ptfl} 1 \mathrm{a}^{\text {cre/+}}$; LSL$\mathrm{Kras}^{\mathrm{G} 12 \mathrm{D}}$; Tgfbr2 ${ }^{\mathrm{fl} / \mathrm{fl}}$ mice enabling facile visualization of tumors among the surrounding normal tissue. ${ }^{18} \mathrm{~F}$-VUIIS-1018 uptake was three-fold higher in tumor tissues compared to adjacent non-tumor tissue and was in concordance with increased TSPO expression in the tumor as measured by immunohistochemistry. We are currently exploring the feasibility of TSPO ligand PET to detect early lesions and will determine the suitability of advancing a novel TSPO ligand to patients with pancreatic cancer. Ongoing preclinical imaging studies also include determining the suitability of TSPO ligand PET to monitor disease progression and to predict response to therapies currently being evaluated at our institution.

\section{3}

Synthesis of benzimidazoles bearing oxadiazole and triazolo-thiadiazole nucleus as new anticancer agents. R. Mohd, Sr., H. Asif, M. Ravinesh; Jamia Hamdard University, New Delhi, India

Cancer is a disease of the cell cycle, in which abnormal cells divide by mitosis without control, and has been one of the major health problems in the world for decades. There is an urgent need for novel effective drug regimens for the treatment of cancer because the current chemotherapy suffers from a slim therapeutic index, with significant toxicity. A lot of chemical classes of heterocyclic and fused heterocyclic compounds have been identified through molecular biology, empirical screening and rational drug development for evaluation of antitumor agents during the past decades. Due to the above, it was thought worthwhile to prepare new hybrids that comprise benzimidazole nucleus with oxadiazole as well as thiadiazole ring to produce new promising anticancer agents. The structures assigned to all the synthesized hybrids were supported by the results of elemental analysis as well as IR, ${ }^{1} \mathrm{H}$ NMR, ${ }^{13} \mathrm{C}$ NMR and mass spectral data and found in full agreement with the proposed structures. The anticancer activity of synthetic hybrids was screened at National Cancer Institute (NCI), USA, against full NCI 60 cell line panel representing nine human systems as leukemia, melanoma and cancers of lung, colon, brain, breast, ovary, kidney and prostate in accordance with their applied protocol (used $\mathrm{SRB}$ assay). The result is given in three calculated response parameters, GI50 (growth inhibitory activity), TGI (cytostatic activity) and LC50 (cytotoxic activity). The hybrid (Ib-A3k) showed remarkable growth inhibition at a single dose $(10 \mu \mathrm{M})$ and further selected for anticancer screening at five dose concentrations $(0.01,0.1,1,10$ and $100 \mu \mathrm{M})$ against full NCI 60 cell panel, with GI50 values between 0.49 and $48.0 \mu \mathrm{M}$, except HCT-15 (GI50>100), under sensitive range an outstanding activity on all the tested cell lines representing nine different subpanels. With regard to the sensitivity against some individual cell lines the compound showed high activity against non-small cell lung cancer cell lines like HOP-92 (GI50, 0.49; TGI, 19.9; LC50, >100; Log10GI50, -6.30; Log10TGI, -4.70; $\log 10 \mathrm{LC} 50,>-4.00)$. The compound proved to be sensitive towards all the tested leukemia cancer cell lines, with not more than $4.94 \mu \mathrm{M}$ concentrations. All the tested prostate cancer cell lines were sensitive, with not more than GI50 $5.40 \mu \mathrm{M}$ concentrations of the tested compound. The highest growth inhibitory activity was observed against the non-small cell lung cancer HOP-92 cell line, with GI50 value of $0.49 \mu \mathrm{M}$, and minimum growth inhibitory activity against renal cancer ACHN cell line, with GI50 value of $48.0 \mu \mathrm{M}$. Based on these observations, compound Ib-A3k could be a subject 
of further investigations for producing potential antitumor agents. Finally, it is conceivable that further derivatization of such compounds will be of interest with the hope to produce more selective and potential antitumor agents.

\section{4}

Maximizing tumor drug delivery of antibody scaffolds. R. Muchekehu ${ }^{1}$, D. Liu ${ }^{1}$, A. Kaspar ${ }^{1}$, S. Pirie-Shepherd ${ }^{2}$, J. Coronella ${ }^{1} ;{ }^{1}$ CovX-Pfizer, San Diego, CA, ${ }^{2}$ Pfizer, La Jolla, CA

Poor drug concentration, distribution and retention pose significant obstacles to effective therapy of solid tumors. The aim of this study is to understand the optimal parameters of a biotherapeutic, in terms of binding affinity, avidity, molecular mass and pharmacokinetics, to allow maximal drug delivery. Biodistribution was measured using an FGFR4 targeting CovX-body (a nontargeting IgG, covalently linked to an FGFR4-targeting peptide) (150 kDa), and enzymatically generated $\mathrm{F}(\mathrm{ab})_{2}(100 \mathrm{kDa})$ and $\mathrm{Fab}(50 \mathrm{kDa})$. Tumor and normal tissue uptake was measured by imaging tumor uptake of near infra-red conjugated constructs and by measuring tumor and normal tissue levels by binding ELISA. Although peak tumor levels were achieved in 1-2 hrs for the Fab and $\mathrm{F}(\mathrm{ab})_{2}$ versus $8 \mathrm{hrs}$ for the IgG, the percentage of injected dose in tumors was only $0.45 \%, 0.5 \%$, and $2.5 \%$ respectively and $0.3 \%, 2 \%$, and $6 \%$ of the non-targeting controls. The accumulation of the non-targeted scaffolds is due to the enhanced permeability and retention effect observed in tumors, and lower levels of the targeting constructs is likely a result of receptor binding, internalization and degradation. To explore the contribution of avidity, constructs were generated with homodimer peptides, creating $\operatorname{IgG}$ and $\mathrm{F}(\mathrm{ab})_{2}$ with 4 peptides and Fab with 2 peptides. Increased valence resulted in 10 -fold increase in cell-surface binding of the $\mathrm{IgG}$. However, there was an inverse relationship between avidity and intratumoral drug concentration, most likely due to targeted consumption. In vitro, FGFR4-targeting IgG constructs internalized rapidly. Although a proposed barrier effect model suggests this might decrease efficacy, it instead translated into superior efficacy for the homodimer peptide IgG in comparison to the monomer peptide IgG. In a single dose study PK is the most important driver of tumor uptake and maximal drug delivery and minimal normal tissue distribution is seen with the IgG (CovX-body). The Fab and $\mathrm{F}(\mathrm{ab})_{2}$ constructs diffuse faster into the tumor and after an equal exposure higher levels are likely to be achieved. Increasing the avidity leads to lower quantitative levels likely due to increased internalization, but higher efficacy.

\section{5}

Visualizing hypoxia-mediated metabolic heterogeneity within a head and neck squamous cell carcinoma xenograft model. E.C. Nakajima, W. Hou, S. Thorne, E.C. Wiener, S.M. Thomas, J.R. Grandis, B. Van Houten; University of Pittsburgh, Pittsburgh, PA

This study aimed to visualize metabolically distinct populations in vivo in a head and neck squamous cell carcinoma (HNSCC) xenograft model. We hypothesized that the irregular vascularization that develops in tumors leads to metabolic heterogeneity in the tumor due to differential oxygenation. We expect that hypoxic cells have reduced metabolic flexibility due to a diminished mitochondrial oxidative capacity and a concomitant reliance upon glycolysis for ATP production. To validate metabolic flexibility in HNSCC, hypoxia-inducible-factor- $1 \alpha$ (Hif- $1 \alpha)$-induced alterations in metabolism were assessed in two cell lines, Cal33 and OSC19. Cobalt chloride $\left(\mathrm{CoCl}_{2}\right)$ prevents the degradation of Hif- $1 \alpha$, thereby acting as a hypoxia mimic. We have found that glucose consumption was significantly greater by 1.5 -fold in Cal33, but not OSC19 cells, when incubated in $100 \mu \mathrm{M}$ $\mathrm{CoCl}_{2}$-DMEM for 48 hours. Cellular oxidative and glycolytic capacity were compared between $\mathrm{CoCl}_{2}$-treated and untreated cells using a Seahorse Flux Analyzer, which measures oxygen consumption and extra-cellular acidification as cells are treated with metabolic inhibitors. $\mathrm{CoCl}_{2}$-treated Cal33 cells had a 40 percent decrease in ATP-linked oxygen consumption as compared to untreated cells, while OSC19 cells showed no significant changes in their oxidative rate after $\mathrm{CoCl}_{2}$ treatment. Surprisingly, no significant changes were observed in the rates of glycolysis in either cell

*SNMMI/AACR Young Investigator Award winner. line. These results have been confirmed when cells were grown hypoxia (2 percent oxygen). In vivo investigations of tumor metabolic heterogeneity were performed using subcutaneous Cal33 xenografts in Foxn1 nude mice. To visualize tumor metabolism and hypoxia, tumor-bearing mice were injected with $2 \mathrm{nmol}$ of HypoxiSense ( $670 \mathrm{~nm}$ excitation $/ 685 \mathrm{~nm}$ emission), a marker of tissue hypoxia, 2 nmol AngioSense (750/770), a marker of tissue vasculature, and $10 \mathrm{nmol}$ IRDye-800CW-2deoxyglucose (2-DG, 778/800), a glucose analog. Detection of dye accumulation within the tumors was performed by fluorescent molecular tomography (Visen FMT 2500, PerkinElmer). FMT imaging revealed a greater than 10 -fold variation in 2-DG uptake within the tumors. Analysis of fluorescent dye concentration within five tumors revealed that glucose uptake was 2.4 times higher in hypoxic tumor regions as compared to the tumor as a whole $(\mathrm{p}<0.007)$. Immunohistochemical staining of tumor sections revealed distinct regions of ATP synthase- or Hif- $1 \alpha$-expressing tissue, indicating the presence of hypoxic and highly oxidative populations within the xenograft tumors. In summary, we have identified a distinct metabolic phenotype that results from induction of Hif- $1 \alpha$ in Cal33 cells and we have detected metabolic heterogeneity within a HNSCC xenograft model. Future studies will investigate a link between metabolic heterogeneity and patient outcome.

\section{$56^{*}$}

Phage display selection for improved renal clearance of diagnostic peptides. J.R. Newton-Northup ${ }^{1}$, M.T. Dickerson ${ }^{1}$, G.P. Smith ${ }^{1}$, S.L. Deutscher ${ }^{2} ;{ }^{1}$ University of Missouri, Columbia, MO, ${ }^{2}$ Harry S. Truman VA Hospital, Columbia, MO

Radioimaging with peptide diagnostics is currently hindered by high kidney retention. We hypothesized that bacteriophage (phage) display could be exploited to select for peptide sequences that redirect phage clearance away from the reticuloendothelial system (RES) and towards excretion through the kidney. A fUSE5 15 amino acid random peptide phage display library was selected in vivo in nude mice to identify phage and corresponding peptides that were excreted into the urine within 1 hour post-injection of the library. Comparative analysis of the resulting $23 \mathrm{RCC}$ sequences from the in vivo selection protocol revealed enrichment in the amino acids Ser, Arg, Gly, Val, and Leu. Lys $(0.58 \%$ of total amino acid composition) was noticeably absent from the final round of selected phage clones, presumably because phage displaying peptides with Lys would be trapped in the kidney and not excreted into the urine. Tripeptide consensus sequences generated from the selected phage included HRS/V, PSR, and SRL. A rapid assessment of modified phage biodistribution and kidney retention of the selected renal clearance clones (RCC) was then performed using in vivo optical imaging with AlexaFluor680 (AF680)-labeled phage. Ex vivo imaging of organs at 1.5 hours post-injection of AF680-labeled phage clones revealed that 5 of the phage clones examined displayed peptides that re-directed excretion through the kidney and bladder and demonstrated varying amounts of liver accumulation. Next, eighteen renal clearance peptides (RCP) containing tripeptide consensus sequences were synthesized for further investigation into renal clearance. Near infrared quantum dots (QD) conjugated to streptavidin were preloaded with biotinylated RCP, and utilized in optical imaging analysis of renal clearance. In vivo and ex vivo imaging of the mice and corresponding organs revealed that three RCPs (3, 4, and 14) of the 18 different RCP peptide/QD samples exhibited improved clearance patterns of the QD when compared to the control QD. Pharmacokinetic studies were then performed with four 1,4,7,10-tetraazacyclododecane-1,4,7,10-tetraacetic acid (DOTA) conjugated peptides selected from in vivo optical imaging analysis of AF680-labeled RCC phage (RCP2) and RCP/QD complexes (RCP3, RCP4, and RCP14). RCP2 is a 15 amino acid peptide (AGGLSFGTRRFEIGR) isolated from the in vivo selection which showed favorable AF680-labeled phage clearance. The other three peptides were identified during the analysis of RCP/QD complexes containing tripeptide consensus sequences: RCP3 (AAHRSPSR), RCP4 (AAHRSAHFVSAPSRAA), and RCP14 (AHRVAASRL). These four DOTA conjugate peptides were radiolabeled with ${ }^{111} \mathrm{In}, \mathrm{HPLC}$ purified, and utilized in biodistribution studies in normal CF-1 mice. All four peptides exhibited markedly improved rates of clearance, low kidney uptake, and reduced non-target organ uptake compared to a control tumor-targeting ErbB2-avid ${ }^{111}$ In-DOTA-peptide with the sequence KCCYSL, which exhibits moderate-high kidney uptake. Kidney retention of the ErbB2avid ${ }^{111}$ In-DOTA-peptide at 2 hours post-injection was $5.75 \pm 0.69 \% \mathrm{ID} / \mathrm{g}$. 
In comparison, the four RCPs tested exhibited significantly reduced kidney retention at 2 hours post-injection. ${ }^{111}$ In-DOTA-labeled RCP2, RCP3, $\mathrm{RCP} 4$, and RCP14 had kidney retention values of $1.66 \pm 0.43,1.63 \pm 0.23$, $2.00 \pm 0.24$, and $1.58 \pm 0.52 \% \mathrm{ID} / \mathrm{g}$, respectively. These results suggest that phage display can be employed to identify peptides that, once radiolabeled, can bypass the RES and be rapidly excreted in urine. Future studies will include appending key binding motifs to tumor-targeting peptides such as the ErbB2-avid peptide KCCYSL to improve tumor radioimaging efficacy.

\section{$57^{*}$}

Imaging apoptosis with positron emission tomography: bench-tobedside development of the caspase-3/7-specific radiotracer $\left[{ }^{18} \mathbf{F}\right]$ ICMT-11. Q. Nguyen ${ }^{1}$, A. Challapalli ${ }^{1}$, G. Smith ${ }^{2}$, R. Fortt ${ }^{1}$, M. Glaser ${ }^{3}$, E. $\AA_{\text {Åstad }}^{4}$, M.A. Gudi ${ }^{1}$, L.S. Carroll ${ }^{1}$, M. Perumal ${ }^{1}$, T.H. Witney ${ }^{1}$, M.A Graham $^{5}$, E.O. Aboagye ${ }^{1}$; ${ }^{1}$ Comprehensive Cancer Imaging Centre, Imperial College London, London, United Kingdom, ${ }^{2}$ Post-Graduate Medical Institute, University of Hull, Hull, United Kingdom, ${ }^{3} \mathrm{MDx}$ Discovery (Part of GE Healthcare), The Grove Centre, Amersham, United Kingdom, ${ }^{4}$ University College London, London, United Kingdom, ${ }^{5}$ TetraLogic Pharmaceutical, Malvern, PA

The capacity to evade apoptosis has been defined as one of the hallmarks of cancer and, thus, effective anti-cancer therapy often induces apoptosis. A biomarker for imaging apoptosis could assist in monitoring the efficacy of a wide range of current and future therapeutics. Despite the potential, there are limited clinical examples of the use of positron emission tomography for imaging of apoptosis. Here we report the "bench to bedside" development of $\left[{ }^{18} \mathrm{~F}\right] \mathrm{ICMT}-11$, a novel reagent designed to non-invasively image caspase-3 activation and, hence, drug-induced apoptosis. This includes rational chemical design, radiosynthesis, in vitro and in vivo biological validation and early clinical development. $\left.{ }^{18} \mathrm{~F}\right] \mathrm{ICMT}-11$ was designed as a small molecule radiotracer with potential advantages such as flexible structural modifications, facile radiolabeling and improved biodistribution and clearance profiles. From an isatin 5-sulfonamide scaffold, a focused library of compounds was screened for activated caspase-3 inhibitory activity and ICMT-11 emerged as the lead compound with subnanomolar affinity for activated caspase- $3(0.5$ $\mathrm{nM})$ and reduced lipophilicity. A fully automated radiosynthesis of $\left[{ }^{18} \mathrm{~F}\right]$ ICMT-11 was developed and optimized to achieve high yield (4.6 $40.4 \mathrm{GBq})$, with a radiochemical purity of $>98 \%$ at EOS and a specific activity of $685 \pm 237 \mathrm{GBq} \cdot \mu \mathrm{mol}^{-1}$ within $90 \mathrm{~min}$. The procedure was further developed to enable GMP synthesis. ICMT-11 has been shown to retain caspase-3 inhibitory activity and further experiments using cellular models of anticancer drug induced-apoptosis have highlighted the binding of $\left[{ }^{18} \mathrm{~F}\right] \mathrm{ICMT}-11$ in caspase-3 activated cells undergoing apoptosis. The initial in vivo assessment of $\left[{ }^{18} \mathrm{~F}\right] \mathrm{ICMT}-11$ in small animals indicated favorable biodistribution of the radiotracer, rapid clearance from the blood and no indication of defluorination. Moreover, there was increased tumor retention of $\left[{ }^{18} \mathrm{~F}\right] \mathrm{ICMT}-11$ detected as early as 6 and $24 \mathrm{~h}$ post-treatment with the small molecule IAP inhibitor TL32711 and the alkylating agent cyclophosphamide, respectively. Confirmatory studies indicated that $\left[{ }^{18} \mathrm{~F}\right] \mathrm{ICMT}-11$ tumor retention was associated with tumor apoptosis. The favorable biological validation of $\left[{ }^{18} \mathrm{~F}\right]$ ICMT-11 as a caspase-3 specific radiotracer to image tumor apoptosis prompted its clinical translation. Additional biological characterization comprising in vitro kinase screen, in vitro uptake studies, metabolic stability assays in vitro and in vivo, and in vivo preclinical imaging studies were performed. Further rat dosimetry and toxicity data for prediction of human dosimetry and safety were acquired. Notably, the radiotracer demonstrated suitable rodent dosimetry profiles $\left(0.03 \mathrm{mSv} \cdot \mathrm{MBq}^{-1}\right)$ with a safety window of 2000 -fold the equivalent dose in humans. A biodistribution, radiation dosimetry, and safety study of $\left[{ }^{18} \mathrm{~F}\right] \mathrm{ICMT}-11$ in healthy volunteers has now been completed. Due to the promising mechanistic and safety profile of $\left[{ }^{18} \mathrm{~F}\right]$ ICMT-11, the radiotracer is transitioning to assess chemotherapy induced caspase- 3 activation in breast cancer patients in early 2013. $\left[{ }^{18} \mathrm{~F}\right] \mathrm{ICMT}-11$ has also been selected as a candidate radiotracer by the IMI/QuIC-ConCePT European consortium for further evaluation in preclinical models and humans. A successful outcome will permit use of this reagent as an "off-the-shelf" apoptosis radiotracer for evaluation of the pharmaceutical industry's next generation therapeutics.

*SNMMI/AACR Young Investigator Award winner.
58

Imaging exosome transfer from breast cancer cells to stroma at metastatic sites in orthotopic nude-mouse models. A. Suetsugu ${ }^{1}, \mathrm{~K}$. Honma $^{1}$, S. Saji ${ }^{2}$, H. Moriwaki ${ }^{2}$, T. Ochiya ${ }^{3}$, R.M. Hoffman ${ }^{1} ;{ }^{1}$ AntiCancer Inc., San Diego, CA, ${ }^{2}$ Department of Gastroenterology, Gifu University Graduate School of Medicine, Gifu, Japan, ${ }^{3}$ Division of Molecular and Cellular Medicine, National Cancer Center Research Institute, Tokyo, Japan

Exosomes play an important role in cell-to-cell communication to promote tumor metastasis. In order to image the fate of cancer-cell-derived exosomes in orthotopic nude-mouse model of breast cancer, we used green fluorescent protein (GFP)-tagged CD63, which is a general marker of exosomes. Breast cancer cells transferred their own exosomes to other cancer cells and normal lung tissue cells in culture. In orthotopic nude-mouse models, breast cancer cells secreted exosomes into the tumor microenvironment. Tumor-derived exosomes were incorporated into stromal cells as well as circulating in the blood of mice with breast cancer metastases. These results suggest that tumor-derived exosomes may contribute to forming a niche to promote tumor growth and metastasis. Our results demonstrate the usefulness of GFP imaging to investigate the role of exosomes in cancer metastasis.

\section{9}

Investigation of gene expression patterns associated with ${ }^{18}$ F-fluorocholine PET kinetics in primary liver tumors. G. Okimoto, M. Loomis, A. Zeinalzadeh, T. Wenska, M. Tiirikainen, B. Hernandez, L. Wong, S. Kwee; University of Hawaii Cancer Center, Honolulu, HI

Purpose: Conduct integrated analysis of global gene expression and in-vivo molecular imaging to preliminarily identify genes and signaling pathways potentially associated with altered tissue choline phospholipid metabolism as measured using ${ }^{18} \mathrm{~F}$-fluorocholine (FC) $\mathrm{PET} / \mathrm{CT}$ in primary liver tumors. Methods: Whole-genome expression profiling (BeadChip/cDNA-mediated annealing, selection, extension, and ligation; Illumina) was performed on 15 fresh-frozen tissue samples obtained from tumor and adjacent-normal regions of the liver from 6 patients who underwent dynamic ${ }^{18} \mathrm{~F}$-fluorocholine PET/CT of the liver prior to tumor resection. PET time-activity course data corresponding to tumor and non-tumor regions of interest in the liver were analyzed using a general 2-tissue compartment PET kinetic model and compared with generalized $\log 2$ transform normalized data from 14 expression arrays (6 Normal, 8 Tumor). The feasibility of supervised geneshaving (superGS) to identify genes with expression profiles correlated with various PET kinetic parameters was then tested. Gene sets were evaluated for association with clinical parameters by cluster analysis, and Ingenuity Pathway Analysis (IPA; Ingenuity Systems) was used to functionally profile individual gene sets for enrichment along known biological processes and signaling pathways, including those associated with hepatocellular carcinoma (HCC), liver disease and liver function. Results: The use of superGS identified a set of 95 genes as being significantly correlated with the transport rate parameter $\mathrm{k} 1$ obtained from PET kinetic modeling. Cluster analysis based on these genes partitioned the 14 samples into distinct groups by tumor status (primary liver tumor vs. adjacent-normal), tumor type ( $6 \mathrm{HCC}$ vs. 2 Cholangiocarcinoma), and patient (when multiple tumor samples were available). Tumor samples exhibited greater heterogeneity in expression compared to normal liver tissue, potentially reflecting a higher degree of phenotypic heterogeneity in tumor relative to non-tumor samples. Functional profiling using IPA confirmed significant enrichment for genes associated with $\mathrm{HCC}(15$ genes, $\mathrm{p}=2.9 \mathrm{E}-07)$, Hepatic System Disease (29 genes, $\mathrm{p}=4.36 \mathrm{E}-09)$, Liver Cholestasis ( 9 genes, $\mathrm{p}=8.3 \mathrm{E}-09)$, Fatty Acid Metabolism Pathway (11/119 genes, $\mathrm{p}=2.03 \mathrm{E}-11)$, Lipid Metabolism (43 genes, $\mathrm{p}=3.3 \mathrm{E}-13)$, and FXR/RXR Pathway $(14 / 101$ genes, $p=8.08 \mathrm{E}-17$ ). Potential upstream regulators of genes correlated with FC influx included NR1H4 ( $\mathrm{p}=3.0 \mathrm{E}-17), \mathrm{FXR} / \mathrm{RXR}$ Complex ( $\mathrm{p}=5.98 \mathrm{E}-13)$, HNF4A ( $\mathrm{p}=1.06 \mathrm{E}-12)$, PPARA (5.42E-12) and HNF1A (4.05E-09), all of which have been reported by other investigators as being associated with $\mathrm{HCC}$ or liver disease. The predicted downstream effects on the k1-associated genes included: Liver Cancer (15 genes, $\mathrm{p}=1.0 \mathrm{E}-07)$, Cholestasis $(9$ genes, $\mathrm{p}=1.0 \mathrm{E}-08)$, Hepatic Steatosis ( 9 genes, $\mathrm{p}=1.0 \mathrm{E}-05$ ), and Cholangiocarcinoma ( 4 genes, $\mathrm{p}=1.0 \mathrm{E}-04$ ). Conclusion: This preliminary study in a limited number of patients suggests that altered hepatic choline phospholipid metabolism, as measured in-vivo using FC PET/CT, may be associated with a number of biological processes and signaling pathways implicated in the pathogenesis of $\mathrm{HCC}$ and liver disease. 
ImmunoPET imaging of prostate stem cell antigen (PSCA) in pancreatic xenografts: comparison of intact IgG with engineered antibody fragments. T. Olafsen ${ }^{1}$, E. Cabral $^{1}$, L. Rahib ${ }^{1}$, J. Keppler ${ }^{1}$, C.P. Behrenbruch $^{1}$, R.E. Reiter ${ }^{2}$, A.M. Wu ${ }^{2}$, J.M. Gudas ${ }^{1}$, D.T. Ho ${ }^{1} ;{ }^{1}$ ImaginAb Inc., Inglewood, CA, ${ }^{2}$ University of California Los Angeles, Westwood, CA

Background: Early diagnosis and efficient therapeutic intervention remain a major challenge in the clinical management of pancreatic cancer patients. Radiolabeled antibodies and engineered antibody $(\mathrm{Ab})$ fragments such as the minibody $\left(\mathrm{scFv}^{-\mathrm{C}_{\mathrm{H}}} 3 ; \sim 80 \mathrm{kDa}\right)$ and Cys-diabody ( $\mathrm{scFv}$ dimer; $\sim 50 \mathrm{kDa}$ ) provide the desired specificity for recognizing cell surface antigens. These platforms retain similar binding affinity and specificity to antibodies with the added advantage of improved pharmacokinetics and clearance optimized for targeted diagnostic imaging. The humanized parental 2B3 mAb (1), specific for prostate stem cell antigen (PSCA), was affinity matured and reformatted into a minibody (A11 Mb) that was validated previously in prostate and pancreatic cancer models (2). In this study the A11 diabody with a terminal cysteine (Cys- $\mathrm{Db})$ was generated and compared with the $\mathrm{A} 11 \mathrm{Mb}$ and parental $2 \mathrm{~B} 3$ in vivo to determine their suitability as imaging agents in pancreatic cancer. Methods: 2B3, A11 Mb and Cys-Db were radioiodinated with I-124 ( $\mathrm{t}_{1 / 2} 4.2$ days) using the Iodogen method. Groups of 4-5 nude, female mice harboring Capan-1 (low PSCA expresser) and MIA-PaCa2 (negative control) tumors were injected with $140 \mu \mathrm{Ci}(\mathrm{Cys}-\mathrm{Db})$ or $100 \mu \mathrm{Ci}(\mathrm{Mb}$ and $2 \mathrm{~B} 3)$ and serially imaged by $\mathrm{PET} / \mathrm{CT}$. One mouse from each group was subjected to a $0-2$ hour dynamic image and one representative mouse was imaged with FDG to confirm viability of the tumors. After the last scan, mice were sacrificed and tumor, blood and organs were harvested and the percent injected dose per gram $(\% \mathrm{ID} / \mathrm{g})$ was determined. Regions of interest (ROIs) were drawn over the heart and tumors to calculate blood clearances and tumor uptakes at the different scan times. Results: The radiochemical purities after radioiodination were $\sim 90 \%$, the specific activities were $\sim 5 \mu \mathrm{Ci} / \mu \mathrm{g}$ and cellbased immunoreactivities ranged from $34-75 \%$. FDG scans confirmed that the tumors were viable. Specific targeting to Capan-1 xenografts was observed with all three constructs. Clear delineation of the tumors was seen as early as 2 hours with the $\mathrm{A} 11 \mathrm{Cys}-\mathrm{Db}$, and at 24 hours with the $\mathrm{A} 11 \mathrm{Mb}$ and 2B3. ROI analyses revealed rapid clearance of the A11 Cys-Db with most of the blood activity gone by $8 \mathrm{hrs}$. The $\mathrm{A} 11 \mathrm{Mb}$ exhibited intermediate blood clearance with most of the activity gone by $24 \mathrm{hrs}$ while $2 \mathrm{~B} 3$ showed a slow clearance profile consistent for intact antibodies. The elimination phase $\left(\mathrm{t}_{1 / 2 \beta}\right)$ was determined to be $3.63,8.66$, and $80.45 \mathrm{hrs}$ for the A11 Cys- $\mathrm{Db}, \mathrm{A} 11 \mathrm{Mb}$ and $2 \mathrm{~B} 3$, respectively. ROI analyses revealed a positive tumor to blood ratio of 9.5 for the A11 Cys-Db at $24 \mathrm{hrs}$ which was 7-fold over that of the $\mathrm{A} 11 \mathrm{Mb}$ and 15 -fold over that of the 2B3. Biodistribution analyses revealed a positive tumor to negative tumor ratio of 5.7 for A11 Cys- $\mathrm{Db}$ at $24 \mathrm{hrs}$. The $\mathrm{A} 11 \mathrm{Mb}$ had similar tumor to negative tumor ratio (5.8) at 48 hrs. Conclusion: Specific targeting and high contrast images were obtained with the ${ }^{124} \mathrm{I}$-labeled A11 Cys-Db and $\mathrm{Mb}$ in a pancreatic xenograft with low expression of PSCA. The A11 Cys-Db exhibited improved properties over the $\mathrm{A} 11 \mathrm{Mb}$ and $2 \mathrm{~B} 3$ as an imaging agent and yielded high contrast images at an earlier time point. When considered together these data suggest that A11 Cys-Db may be a promising agent for early imaging of pancreatic cancer. We are currently investigating the properties of A11 Cys- $\mathrm{Db}$ and $\mathrm{Mb}$ in a high expressing PSCA pancreatic tumor model. References: 1. Olafsen, $T$ et al. J Immunother 2007;30(4):396-405. 2. Lepin, EJ et al. Eur J Nucl Med Mol Imaging. 2010;37(8):1529-38

\section{$61^{*}$}

$\left[{ }^{18}\right.$ F]CAIP: a novel PET tracer for imaging caspase-3-initiated apoptosis in treated tumors. M. Palner, B. Shen, J. Jeon, D. Ye, A. Shuhendler, F.T. Chin, J. Rao; Department of Radiology, Stanford University Medical Center, Stanford, CA

Monitoring the effect of treatment in cancer patients is vital to improving their survival rate. Current cancer treatments including radiation therapy and chemotherapy (e.g. doxorubicin), ultimately induce apoptosis (programmed cell death) in the targeted tumor cells. One of the most important pathways of therapy-induced apoptosis requires the activation of caspase-3, a central scavenging peptidase which cleaves a specific protein sequence DEVD-X (Asp-Glu-Val-Asp-Xxx) between D and X, committing the cell to programmed cell death. Imaging agents that enable direct visualization of active caspase- 3 in cancer patients have great potential value for maintaining effective treatment in the clinic. We designed a novel caspase-3-activatable tracer $\left(\left[{ }^{18} \mathrm{~F}\right] \mathrm{CAIP}\right)$ for PET imaging of apoptosis. Pro $\left[{ }^{18} \mathrm{~F}\right] \mathrm{CAIP}$ contains the DEVD-C peptide substrate that is cleaved specifically by caspase- 3 . Upon cleavage, pro $\left[{ }^{18} \mathrm{~F}\right] \mathrm{CAIP}$ undergoes an intramolecular cyclization reaction, transforming the hydrophilic pro-tracer into the hydrophobic $\left[{ }^{18} \mathrm{~F}\right] \mathrm{CAIP}$. These hydrophobic monomers are prone to forming large aggregates in situ that result in intracellular trapping of the probe, enhancing tumor accumulation and increasing signal-to-noise ratio in apoptotic cells. Pro $\left[{ }^{18} \mathrm{~F}\right] \mathrm{CAIP}$ has been synthesized with $5.6 \pm 3.7 \%$ decay-uncorrected radiochemical yield and $>99 \%$ radiochemical purity, and a specific activity of $1.75 \pm 0.72 \mathrm{mCi} / \mathrm{nmol}(\mathrm{n}=6)$. In vitro intramolecular cyclization of $\left.{ }^{18} \mathrm{~F}\right] \mathrm{CAIP}$ after incubation with recombinant caspase- 3 has been verified by HPLC, and experiments with HeLa cells $(n=3)$ demonstrated a two-fold increase in tracer uptake in apoptotic cells compared with untreated control. Dynamic microPET scanning (60 min. dynamic $+5 \mathrm{~min}$. static at $2 \mathrm{~h}, 447 \pm 6.7 \mu \mathrm{Ci} /$ mice i.v.) of four $\mathrm{HeLa}$ tumor bearing mice after treatment with doxorubicin $(0.2 \mathrm{mg}$, intratumoral $)$ showed high uptake in the apoptotic tumor $(4.18 \pm 0.32 \% \mathrm{ID} / \mathrm{g})$, reaching plateau after $22 \mathrm{~min}$. The kidneys showed the highest uptake $(30.5 \pm 0.32$ $\% \mathrm{ID} / \mathrm{g}$ ) after $60 \mathrm{~min}$, suggesting clearance through the renal system. The measured uptake of $\left[{ }^{18} \mathrm{~F}\right] \mathrm{CAIP}$ was confirmed after 2 hour post-injection by performing a static microPET scan followed by ex vivo biodistribution analysis. In another cohort of similar mice, we compared the uptake of $\left[{ }^{18} \mathrm{~F}\right]$ CAIP before and after treatment in the same mice ( 5 min. static scanning at $1,2$ and $3 \mathrm{~h}, 235 \pm 10 \mu \mathrm{Ci} /$ mice i.v. $)$, and found a significant $(\mathrm{p}<0.05)$ twofold enhancement in tumor uptake following treatment with doxorubicin (0.2 mg, intratumoral). [ $\left.{ }^{18} \mathrm{~F}\right] \mathrm{CAIP}$ shows promise as a caspase-3-activatable tracer for monitoring apoptosis as a surrogate of tumor treatment response during cancer chemotherapy. We believe that such a PET tracer will be able to reduce ineffective treatment for cancer patients in the future, thereby improving patient quality of life and treatment outcomes.

\section{2}

PET imaging of uPAR expression using ${ }^{64} \mathrm{Cu}$-labeled cross-bridged cyclam-based ligands for noninvasive identification of invasive cancers: a comparative study. M. Persson ${ }^{1}, \mathrm{M}$. Hosseini ${ }^{2}$, J. Madsen ${ }^{1}$, K.J. Jensen ${ }^{2}$, A. Kjaer ${ }^{1}$, M. Ploug ${ }^{3} ;{ }^{1}$ Cluster for Molecular Imaging, Dept. of Nuclear Medicine and PET, Rigshospitalet, Copenhagen, Denmark, ${ }^{2}$ Nanobioscience Group, Dept. of Basic Sciences, Faculty of Life Sciences, University of Copenhagen, Copenhagen, Denmark, ${ }^{3}$ Finsen Laboratory and BRIC, Rigshospitalet, University of Copenhagen, Copenhagen, Denmark

Introduction and Aim: The correlation between uPAR expression, cancer cell invasion and metastases is well-established and has prompted the development of a number of uPAR PET imaging agents (1-3) which could potentially identify cancer patients with invasive lesions. In the present study, we synthesized and characterized two cross-bridge based ${ }^{64} \mathrm{Cu}$-labeled peptide conjugates for PET imaging of UPAR and performed a head-to-head comparison with the corresponding DOTA conjugate. Method: The UPARtargeting peptide (AE105) was synthesized using standard Fmoc solidphase peptide chemistry, conjugated to DOTA, CB-TE2A or a CB-TE2A-analogue carrying one additional propionic acid linker (CB-TE2A-PA), and finally radiolabeled with ${ }^{64} \mathrm{Cu}$. All peptides were evaluated for their binding and stability properties in vitro, as well as for their biodistribution profiles in vivo in a human glioblastoma U87MG xenograft mouse model using microPET/CT and gamma-counting. Results: No reduction in the uPAR receptor binding affinity of the targeting peptide was encountered by conjugation to either of the three macrobicyclic chelators $\left(\mathrm{IC}_{50} \sim 5-10 \mathrm{nM}\right)$ and high yields and radiochemical purities $(>95 \%)$ were achieved in all cases by incubation at $95^{\circ} \mathrm{C}$. In vivo, identical tumor uptake after $1 \mathrm{~h}$ was found, but significantly different tumor uptake $22 \mathrm{~h}$ post injection (p.i.) based on both PET/CT studies and gamma-counter based biodistribution studies, with ${ }^{64} \mathrm{Cu}-\mathrm{DOTA}-\mathrm{AE} 105,{ }^{64} \mathrm{Cu}-\mathrm{CB}-\mathrm{TE} 2 \mathrm{~A}-$ $\mathrm{AE} 105$ and ${ }^{64} \mathrm{Cu}-\mathrm{CB}-\mathrm{TE} 2 \mathrm{~A}-\mathrm{PA}-\mathrm{AE} 105$ having a tumor uptake of $4.5 \pm 0.9$, 
$2.0 \pm 0.1$ and $2.6 \pm 0.3 \% \mathrm{ID} / \mathrm{g}(\mathrm{p}<0.01)$. A significantly reduced liver uptake was furthermore observed with ${ }^{64} \mathrm{Cu}-\mathrm{CB}-\mathrm{TE} 2 \mathrm{~A}-\mathrm{PA}-\mathrm{AE} 105$ compared with ${ }^{64} \mathrm{Cu}-\mathrm{DOTA}-\mathrm{AE} 105$ and ${ }^{64} \mathrm{Cu}-\mathrm{CB}-\mathrm{TE} 2 \mathrm{~A}-\mathrm{AE} 105(\mathrm{p}<0.0001)$, indicating superior in vivo stability. Finally, the specificity of the best performing ligand, ${ }^{64} \mathrm{Cu}-\mathrm{CB}-\mathrm{TE} 2 \mathrm{~A}-\mathrm{PA}-\mathrm{AE} 105$, was confirmed in vivo using a nonbinding ${ }^{64} \mathrm{Cu}$-labeled peptide as control $\left({ }^{64} \mathrm{Cu}\right.$-CB-TE2A-PA-AE105mut), where a significantly reduced tumor uptake was found compared with ${ }^{64} \mathrm{Cu}-\mathrm{CB}-\mathrm{TE} 2 \mathrm{~A}-\mathrm{PA}-\mathrm{AE} 105$ both after 1 and $22 \mathrm{~h}$ p.i. $(\mathrm{p}<0.0001)$. Conclusion: Based on our head-to-head PET comparison, ${ }^{64} \mathrm{Cu}-\mathrm{CB}-\mathrm{TE} 2 \mathrm{~A}-$ PA-AE105 proved to have superior in vivo stability and a high and specific tumor uptake, which translates into a significantly higher tumor-to-liver ratio compared with ${ }^{64} \mathrm{Cu}$-DOTA-AE105 and ${ }^{64} \mathrm{Cu}-\mathrm{CB}-\mathrm{TE} 2 \mathrm{~A}-\mathrm{AE} 105$. This new PET ligand is thus setting the stage for achieving an excellent contrast during non-invasive PET imaging of uPAR expression in cancer patients. References: 1. Li ZB, Niu G, Wang H, et al. Imaging of urokinase-type plasminogen activator receptor expression using a ${ }^{64} \mathrm{Cu}$-labeled linear peptide antagonist by microPET. Clin Cancer Res. Aug 1 2008;14(15):4758-4766. 2. Persson M, Madsen J, Ostergaard S, et al. Quantitative PET of human urokinase-type plasminogen activator receptor with ${ }^{64} \mathrm{Cu}$-DOTA-AE105: implications for visualizing cancer invasion. J Nucl Med. Jan 2012;53(1): 138-145. 3. Persson M, Madsen J, Ostergaard S, Ploug M, Kjaer A. ${ }^{68} \mathrm{Ga}$-labeling and in vivo evaluation of a uPAR binding DOTA- and NODAGA-conjugated peptide for PET imaging of invasive cancers. Nucl Med Biol. May 2012;39(4):560-569.

\section{3}

FDG-PET FDG findings in Kaposi sarcoma herpes virus-associated multicentric Castleman disease (KSHV-MCD) correlate with clinical, inflammatory, and virologic disease parameters. M.N. Polizzotto ${ }^{1}$, C. Millo $^{2}$, T.S. Uldrick ${ }^{1}$, K. Aleman ${ }^{1}$, K. Wyvill ${ }^{1}$, M. Whatley ${ }^{2}$, D. O'Mahony ${ }^{1}$, V. Marshall ${ }^{3}$, D. Whitby ${ }^{3}$, S. Steinberg ${ }^{1}$, R. Little ${ }^{1}$, R. Yarchoan ${ }^{1},{ }^{1}$ National Cancer Institute, Bethesda, MD, ${ }^{2}$ National Institutes of Health, Bethesda, MD, ${ }^{3}$ Frederick National Laboratory for Cancer Research, Frederick, MD

Background: KSHV-associated multicentric Castleman disease (KSHVMCD) is a virally associated lymphoproliferative tumor associated with severe inflammatory symptoms, cytopenias and biochemical abnormalities resulting at least in part from KSHV lytic activation and consequent immune abnormalities. It is most common in individuals with HIV. Improved techniques to assist diagnosis and aid monitoring, including assisting in distinction from lymphoma or manifestations of HIV alone, are required. We prospectively assessed ${ }^{18} \mathrm{FDG}-\mathrm{PET} / \mathrm{CT}$ findings in KSHV-MCD in relation to clinical symptoms and markers of disease activity. Methods: As a prospectively specified objective, patients enrolled on a natural history study of KSHV-MCD (NCT00099073) underwent ${ }^{18}$ FDG-PET/CT at disease activity, except where unstable, and at complete clinical and biochemical remission. ${ }^{18} \mathrm{FDG}-\mathrm{PET} / \mathrm{CT}$ was evaluated blind to clinical status. Symptoms, C-reactive protein (CRP), HIV viral load (VL) in plasma and KSHV VL in peripheral blood mononuclear cells were assessed; all of these parameters except HIV VL are known markers of KSHV-MCD disease activity. Associations with ${ }^{18} \mathrm{FDG}-\mathrm{PET} / \mathrm{CT}$ maximal standardized uptake value (SUVmax) were explored using Spearman correlations (CRP, symptoms, $\log _{10}[$ KSHV VL]) or exact Wilcoxon rank sum (HIV VL, detectable or not). Results: 26 patients ( 24 male, median age 43 [range 34-56], all with HIV) were studied. In 3 , we identified intercurrent lymphoma; these were excluded from the primary analysis. The remaining 23 underwent 19 studies during disease activity (16 symptomatic, 3 with laboratory manifestations only), and 21 studies at remission. In symptomatic patients, ${ }^{18}$ FDG-PET showed symmetrical hypermetabolic adenopathy (diffuse in 15 [94\%], focal in 1 [6\%]) and increased splenic metabolic activity with splenomegaly (abnormal in 14 [93\%] of the 15 with intact spleens). Marrow and hepatic abnormalities were less common and mild. In patients with laboratory manifestations only, $2(66 \%)$ had mild splenomegaly and limited adenopathy and $1(33 \%)$ isolated adenopathy. During disease activity, median SUVmax was $6(2-8)$, and was associated with symptom severity $(\mathrm{R}=0.61 \mathrm{p}=0.005), \mathrm{CRP}(\mathrm{R}=0.54, \mathrm{p}=0.017)$ and KSHV VL $(\mathrm{R}=0.56$, $\mathrm{p}=0.013)$, but not HIV VL $(\mathrm{p}=0.69)$. Intercurrent lymphomas (2 PEL and 1 diffuse large B-cell) demonstrated intensely hypermetabolic abnormalities involving restricted asymmetrical sites, clearly distinct from KSHV-MCD only cases, with median SUVmax 11 (range 7-38). In addition in $3(38 \%)$ of 8 patients with Kaposi sarcoma in the field of view, corresponding mildly hypermetabolic skin lesions were seen. At remission, 11 (53\%) had normal ${ }^{18}$ FDG-PET/CT; 10 (47\%) had minor nodal abnormalities and 4 (19\%) mildly increased splenic metabolism without splenomegaly. Intercurrent pathologies contributed to some abnormalities. One patient had progressive increase in splenic and nodal SUVmax over 3 scans (not included in primary analysis) over 12 months after primary chemotherapy before relapse. Conclusion: ${ }^{18} \mathrm{FDG}$-PET/CT demonstrated widespread nodal and splenic abnormalities during KSHV-MCD disease activity, improving with remission. Subclinical disease may also be detectable. Findings were distinguishable from suppressed HIV or intercurrent lymphoma by intermediate metabolic intensity and diffuse symmetrical anatomic distribution. $\mathrm{SUV}_{\max }$ increased in association with worsening symptom severity, systemic inflammation, and increasing KSHV viral activity. ${ }^{18}$ FDG-PET/CT may be a useful non-invasive adjunct in the diagnosis of KSHV-MCD (including guiding biopsy site selection and assisting in excluding other diagnoses) and in the monitoring of pathologically confirmed KSHV-MCD. Exploration of its utility in other KSHV-associated tumors may be warranted.

\section{4}

Suppression of Akt/mTOR pathway in cervical cancer cell line C33A using allosteric Akt inhibitors. R. Ramachandran, J.K. Schwarz; Washington University School of Medicine, St. Louis, MO

Background: Akt signaling is frequently found to be de-regulated in human cancers. We have previously shown that overexpression of pAkt is associated with decreased progression free survival after chemoradiation in human cervical cancer. PI3K/Akt/mTOR pathway inhibitors are currently being evaluated in clinical trials for cancers in other sites. The purpose of this study was to evaluate the effects of two allosteric Akt inhibitors, SC-66 and MK-2206 in human cervical cancer cell lines. Experimental Design: C33A cells were treated with the allosteric Akt inhibitors SC-66 $(0.001 \mu \mathrm{g} / \mathrm{ml}-5 \mu \mathrm{g} / \mathrm{ml})$ and MK-2206 $(250 \mathrm{nM}-30 \mu \mathrm{M})$ with or without the glucose analogue (2-DG) 2-deoxyglucose (5-20mM) using dose titration and time courses to test viability using Alamar Blue. Phosphorylation of Akt and downstream targets of Akt and mTOR pathway with or without SC-66 $(6-10 \mu \mathrm{g} / \mathrm{ml})$ and MK-2206 $(0-2.5 \mu \mathrm{M})$ were determined by western blot. Percent apoptosis was determined by flow cytometry using Annexin-7-AAD staining (BD Biosciences) $24 \mathrm{~h}$ post treatment. Wound healing assay was performed to assess their effects on migration upon SC-66 and MK-2206 treatment. Results: Decreased C33A cell viability was observed using both SC-66 $(1 \mu \mathrm{g} / \mathrm{ml}-56 \%, 43 \%$ and $5 \mu \mathrm{g} / \mathrm{ml}-15 \%$ and $2 \%$ by 24 and $48 \mathrm{~h}$ respectively) and MK-2206 $(5 \mu \mathrm{M}-80 \%, 78 \%$ and $30 \mu \mathrm{M}-49 \%$ and $2 \%$ by 24 and $48 \mathrm{~h}$ respectively) in a dose and time dependent manner. Annexin staining was used to determine whether cell death was occurring by apoptosis. SC-66 treatment resulted in very few cells with Annexin only stain (16.5\%) and the fraction of cells with both staining was 34\%. MK-2206 treated cells showed $5 \%$ Annexin positivity and the percentage of cells with both Annexin and 7-AAD was $12 \%$. 7-AAD only staining was close to $80 \%$ in MK-2206 treated cells thus indicating that cells are dying through a non-apoptotic pathway. Treatment with SC-66 resulted in de-phosphorylation of pAkt and mTORC1 downstream targets $\mathrm{p} 70 \mathrm{~s} 6 \mathrm{~K}$ and 4E-BP1. MK-2206 exhibited a very modest inhibition on the Akt substrates. All the three forms of $\mathrm{p}-\mathrm{Akt}$ were inhibited with partial reactivation of the Thr450 form by $4 \mathrm{~h}$. MK-2206 inhibited the mTORC1 substrate 4E-BP1 completely. The inhibitory effect of MK-2206 on p70s6K was transient as evidenced by rebound Akt activation by $4 \mathrm{~h}$. The combination of 2-DG and MK2206 was found to kill $\mathrm{C} 33 \mathrm{~A}$ cells in a dose and time dependent manner $(5 \mu \mathrm{M}$ MK-2206 only$81 \%$ and $5 \mu \mathrm{M}$ MK-2206 plus 2-DG-50\%). Further we found that MK-2206 inhibited glucose uptake but not SC-66 in C33A cells. SC-66 was found to partially inhibit glut 4 migration from the cytosol to membrane. The wound healing assay showed a substantial reduction in wound healing capacity of C33A cells upon SC-66 treatment whereas there was no noticeable effect with MK-2206. Conclusions: SC-66 and MK-2206 potently inhibited proliferation of cervical cancer cell line C33A by nonapoptotic mechanism. SC-66 resulted in inhibition of phosphorylation of Akt and its substrates along with mTORC1 substrates mTOR, 4EBP1 and p70S6K. SC-66 partially inhibited the translocation of glut4 from cytosol to membrane and also decreased the expression of glut1 in cells. SC-66 also inhibited migration of cells as observed in wound healing assay. MK-2206 displayed synergistic effects with glucose analogue 2-DG and also inhibited 
glucose uptake by C33A. Since high levels of pAkt are associated with poor outcome after concurrent chemo-radiation in cervical cancer, SC-66 and MK-2206 could prove to be effective therapeutic adjuncts in these patients.

\section{5}

Novel bispecific ${ }^{111}$ In-labeled radioimmunoconjugates (bsRICs) for SPECT/CT imaging of EGFR and HER2 receptors in breast cancer. E.J. Razumienko, L. Dryden, R.R. Reilly; University of Toronto, Toronto, ON, Canada

Objective: Our objective was to construct novel bsRICs capable of binding EGFR and HER2 labeled with ${ }^{111}$ In for imaging EGFR-HER2 heterodimerization in breast cancer (BC) possibly through higher avidity for dimeric vs. monomeric complexes. Methods: bsRICs were composed of trastuzumab Fab recognizing HER2 linked to the $6.2 \mathrm{kDa}$ endogenous ligand for EGFR, epidermal growth factor (EGF). Fabs were produced by digestion of trastuzumab $\operatorname{IgG}$ with papain. Fab were modified with Sulfosuccinimidyl-4-(N-maleimidomethyl)cyclohexane-1-carboxylate containing a 24 length polyethyleneglycol (PEG) spacer (SM-PEG $24-\mathrm{NHS}$ ) to introduce maleimide groups for cross-linking to EGF thiolated with Traut's reagent. bsRICs were derivatized with diethylenetriaminepentaacetic acid (DTPA) for labeling with ${ }^{111} \mathrm{In}$. The ability to independently bind HER2 or EGFR was determined in competition assays using ${ }^{111}$ In-bsRICs against unlabeled Fab or EGF on cells expressing HER2, EGFR or both receptors. The tumour and normal tissue uptake of the bsRICs was examined in mice bearing BC xenografts that expressed HER2, EGFR or both receptors, and blocking with Fab and HRG performed to determine specificity for the target receptors. Results: Conjugation of Fab to EGF was confirmed by SDS-PAGE, Western blot and size-exclusion HPLC. bsRICs were labeled with ${ }^{111}$ In to a high radiochemical purity. bsRICs showed specific binding to HER2 and EGFR in cell lines expressing either receptor. In MDA-MB-231 cells (EGFR+/HER2-) binding was reduced to $95.3 \pm$ $7.1 \%, 20.5 \pm 2.6 \%$ or $10.1 \pm 2.8 \%$, respectively by Fab, EGF or both ligands. The binding to SK-OV-3 cells (EGFR-/HER2+) was reduced to $12.6 \pm$ $2.7 \%, 95.9 \pm 8.8 \%$ or $10.1 \pm 1.7 \%$ by Fab, EGF or both ligands. The binding to MDA-MB-231 cells transfected to express HER2 (231-H2N, EGFR+/ HER $2+$ ) was reduced to $52.7 \pm 2.8 \%, 65.5 \pm 2.9 \%$ or $4.4 \pm 0.9 \%$ by Fab, EGF or both ligands. The highest uptake of bsRICs $[7.3 \pm 3.5 \% \mathrm{ID} / \mathrm{g}]$ in 231-H2N human breast cancer xenografts (HER2+/EGFR+) occurred at 48 $\mathrm{h}$ post-injection (p.i.). Pre-administration of trastuzumab Fab decreased uptake in SK-OV-3 (HER2+/EGFR-) human ovarian cancer xenografts from $7.1 \pm 1.2 \% \mathrm{ID} / \mathrm{g}$ to $2.4 \pm 1.5 \% \mathrm{ID} / \mathrm{g}$. Pre-administration of excess EGF decreased uptake in MDA-MB-231 (HER2-/EGFR+) human breast cancer xenografts from $5.9 \pm 0.5 \% \mathrm{ID} / \mathrm{g}$ to $2.0 \pm 0.1 \% \mathrm{ID} / \mathrm{g}$. All tumors were imaged by microSPECT/CT. Conclusion: ${ }^{111}$ In-bsRICs composed of trastuzumab Fab and EGF bound specifically to EGFR and HER2 both in vitro and in vivo. These ${ }^{111}$ In-bsRICs may be useful for imaging EGFR-HER2 heterodimerization in $\mathrm{BC}$, and would prove useful for monitoring response to treatments with HER dimerization inhibitors such as Pertuzumab.

\section{6}

Targeted liposomes for cell death imaging and amplified drug delivery. D.R. Rice, S. Turkyilmaz, R.O. Palumbo, K.M. Harmatys, B.D. Smith; Department of Chemistry and Biochemistry, The University of Notre Dame, Notre Dame, IN

The goal is to develop liposomes that have selective affinity for dead and dying cells, and then use the liposomes as targeted imaging agents for rapidly assessing the efficacy of anticancer therapies. A subsequent application is to use the liposomes to deliver anticancer drugs to tumors and initiate cell death processes that amplify the liposome targeting at later time points in the therapy. The liposome surface is decorated with $2 \% \mathrm{Zn}$-DPAPEG2000-PE, an anchored phospholipid with an extended polyethylene chain that terminates with a zinc-dipicolylamine (Zn-DPA) targeting group. Previous studies with fluorescent Zn-DPA molecular probes have shown that they have high selective affinity for dead and dying cells. Specifically, they target the anionic phospholipid, phosphatidylserine (PS), a cell death biomarker that is exposed on the surface of dead and dying cells. Zn-DPA liposomes are composed of 2\% Zn-DPA-PEG2000-PE, 67\% 1-palmitoyl-2oleoyl-sn-glycero-3-phosphocholine (POPC), 30\% cholesterol and 1\% near-infrared fluorophore (DiR). Untargeted liposomes are composed of $69 \%$ POPC, $30 \%$ cholesterol and $1 \%$ DiR. The ability of Zn-DPA liposomes to target a chemical model of the PS cell death biomarker was assessed using a fluorescence quenching assay. These studies revealed that the PEG chains do not inhibit the affinity of the Zn-DPA units. In vitro fluorescence microscopy of several cancer model cell-lines (MDA-MB-213, Jurkat E6-1) showed that the Zn-DPA liposomes strongly stain dead and dying cancer cells that were treated with the anticancer agent etoposide. Multicolor imaging of dead and dying cells using the small molecule DPA probe PSVue ${ }^{\mathrm{TM}} 480$ demonstrates that Zn-DPA liposomes co-localize to the dead cell periphery, a definitive feature of surface targeting. A rat thymus atrophy model was used to access the in vivo cell death targeting capability of Zn-DPA liposomes. Rats were dosed with intraperitoneal dexamethasone to induce thymocyte cell death. Zn-DPA and untargeted liposomes were intravenously injected into separate cohorts $(n=3)$ and after 24 hours, the rats were euthanized and their organs were excised and imaged. The uptake of targeted liposomes into the dying tissue was more than 4-fold greater than the untargeted liposome system. In summary, liposomes coated with multiple copies of Zn-DPA targeting groups are effective cell death imaging agents for fluorescence microscopy and they can target dead and dying tissue in living animals. With further development, these targeted liposomes have potential utility for rapid clinical evaluation of anticancer drug efficacy in individual patients.

\section{7}

Linking LDH-A, metastases and lactate identified by MRS imaging. I. Serganova, A. Rizwan, R. Khanin, X. Ni, H. Karabeber, K. Zakian, S. Thakur, J.A. Koutcher, R. Blasberg; Memorial Sloan-Kettering Cancer Center, New York, NY

Background: Tumor progression and the development of a metastatic phenotype are closely coupled to metabolic changes in primary tumors. A well-recognized characteristic of patients with aggressive cancer is an elevation in serum Lactate Dehydrogenase LDH, and the upregulation of the gene for LDH-A protein in the tumors (1). High tumor LDH-A expression has been linked to poor prognosis and a greater metastatic potential. LDH-A provides a link between several metabolic pathways involving both glucose and glutamine. Lactate, the product of LDH-A activity, can be assessed noninvasively and quantitatively using magnetic resonance spectroscopic imaging (MRSI). The low sensitivity and overlapping peaks of the lactate and lipid MR spectra present technical difficulties during MR spectroscopy, but these challenges can be overcome by the use of spectral editing methods, such as SELective Multiple Quantum Coherence (SEL-MQC) techniques (2). We investigated the relationship between LDH-A expression and lactate production using lactate MRSI in two isogenic breast cancer lines (3), and showed that small orthotopic 4T1 tumors (high metastatic potential) generate 10-fold more lactate than corresponding 67NR tumors (no metastases). We also showed that Lactate-MRSI had a greater dynamic range than $\left[{ }^{18} \mathrm{~F}\right] \mathrm{FDG}-\mathrm{PET}$ and may be a more sensitive measure with which to evaluate the aggressive and metastatic potential of primary breast tumors. Experimental Design: To further assess the role of LDH-A in the aggressive/metastatic phenotype, we sought to inhibit LDH-A expression in 4T1 breast tumors using LDH-A shRNA knockdown (KD) technology. Stable LDH-A knock-down clones of 4T1 were developed and directly compared to a scrambled-shRNA control cell line (NC). A stable KD clone was selected (KD9), based on LDH-A mRNA expression (qRT-PCR) and LDH-A protein levels (immunoblots), for further in vitro and in vivo studies. We then studied the relationships between KD9 and NC LDH-A expression, lactate concentration, cell metabolism, tumor growth and metastases formation. Results: KD9 cells showed significantly less glucose utilization and glycolysis, lower LDH enzyme activity, less lactate and acid production, and a compensatory increase in oxygen consumption and maximum respiratory capacity, and increased ROS and cellular ATP levels, compared to NC cells. KD9 cells had a lower proliferation rate and decreased transwell migration and invasion than $\mathrm{NC}$ cells. In vivo studies showed that moderate levels of lactate persist in small KD9 tumors, although the levels were significantly less than that in comparable NC and wild-type 4T1 tumors $(\mathrm{p}<0.01)$, and a linear relationship between tumor LDH-A protein expression and tissue lactate concentration (as measured by MRSI) was observed. However KD9 tumor LDH-A silencing was incomplete, and KD9 tumor LDH-A protein and lactate levels gradually increased as KD9 tumors grew to the level observed in NC tumors. Nevertheless, the initial growth 
rate was lower and formation of metastases was delayed in KD9 tumors Conclusions: Inhibition of LDH-A induced primary and metastatic tumor growth delay with enhanced survival, that was accompanied by changes in metabolism (enhanced oxygen consumption and decreased glycolysis). Since elevated LDH-A is a component of many aggressive tumors, it is a potential drug target for cancer therapy $(4,5)$. LDH-A targeted drug therapy may avoid the loss of shRNA-based LDH-A silencing, that we observed during tumor growth and the development of metastases, since LDH-A drug-targeted therapy will have an effect on both tumor and stromal components. Nevertheless, our LDH-A shRNA silencing experiments in a well-studied animal model show a strong association between LDH-A gene and protein expression, tumor lactate levels and the development of metastases. MRSI monitoring can provide a non-invasive imaging strategy to monitor treatment response. References: 1. Koukourakis, M.I., et al., Lactate dehydrogenase-5 (LDH-5) overexpression in non-small-cell lung cancer tissues is linked to tumour hypoxia, angiogenic factor production and poor prognosis. Br J Cancer, 2003. 89(5): p. 877-85. 2. He, Q., et al., Singlescan in vivo lactate editing with complete lipid and water suppression by selective multiple-quantum-coherence transfer (Sel-MQC) with application to tumors. J Magn Reson B, 1995. 106(3): p. 203-11. 3. Serganova, I., et al., Metabolic imaging: a link between lactate dehydrogenase A, lactate, and tumor phenotype. Clin Cancer Res, 2011. 17(19): p. 6250-61. 4. Le, A., et al., Inhibition of lactate dehydrogenase A induces oxidative stress and inhibits tumor progression. Proc Natl Acad Sci U S A, 2010. 107(5): p. 2037-42. 5. Zhou, M., et al., Warburg effect in chemosensitivity: targeting lactate dehydrogenase-A re-sensitizes taxol-resistant cancer cells to taxol. Mol Cancer, 2010. 9: p. 33.

\section{8}

Cyanoquinoline-based tracers and their interaction with the $\mathrm{ABC}$ transporters for the imaging of EGFR in cancer. R. Slade ${ }^{1}, F$. Pisaneschi $^{1}$, M. Kaliszczak ${ }^{1}$, G. Smith ${ }^{2}$, Q. Nguyen ${ }^{1}$, E. Aboagye ${ }^{1}$; ${ }^{1}$ Imperial College London, London, United Kingdom, ${ }^{2}$ Hull University, Hull, United Kingdom

The epidermal growth factor receptor (EGFR) plays a key role in biological processes including proliferation and cell cycle regulation through the activation of downstream signal transduction pathways. Furthermore, this tyrosine kinase receptor is overexpressed in many cancers including ovarian, breast, head and neck and brain. Imaging EGFR using positron emission tomography (PET) linked to EGFR mutational status could help select patients who are more likely to respond to small molecule tyrosine kinase inhibitors such as Gefitinib. We previously showed by PET that ${ }^{18} \mathrm{~F}$-FED6, a radiolabeled cyanoquinoline EGFR antagonist, had higher uptake in A431 xenograft tumors (high EGFR; high ABCG2, no ABCB1) compared to HCT116 xenograft tumors (low EGFR; low ABCG2, no ABCB1). However the overall tumor radiotracer uptake is low possibly due to the ATP binding cassette transporters, frequently overexpressed in tumor cells and actively extruding drugs from the cytosol. The aim of this study is to further characterise cyanoquinoline tracers for ${ }^{18} \mathrm{~F}$ PET imaging of EGFR, investigating the role of the $\mathrm{ABC}$ transporters. Following exposure of isogenic wild-type and $\mathrm{ABC}$ transporter over-expressing cells to the cyanoquinoline series (FED1-9) several of the compounds were found to be potential substrates of ABCB1 (P-glycoprotein) and ABCG2 transporters. Transwell caco 2 cell permeability assays confirmed compound efflux and hence $\mathrm{ABC}$ transporter substrate specificity. Efflux of the cyanoquinolines was significantly decreased in the presence of inhibitors of both ABCB1 and $\mathrm{ABCG} 2$ transporters, further confirming specificity. Radiolabeled ${ }^{18} \mathrm{~F}-$ FED6, was shown to have a four-fold lower in vitro uptake in $\mathrm{ABCB} 1$ or ABCG2 overexpressing cell lines compared to the isogenic counterpart. Finally, in vivo PET imaging in A431 xenografts with ${ }^{18} \mathrm{~F}$-FED6, showed over a two-fold increase in the tumor uptake of the radiolabeled cyanoquinoline, when mice were pre-treated with gefitinib. In vitro studies confirmed gefitinib's role as an ABCG2 inhibitor. We confirm that $\mathrm{ABC}$ transporter expression can confound PET imaging of EGFR using ${ }^{18} \mathrm{~F}$ FED6. Further development and investigation into a second generation of compounds, devoid of $\mathrm{ABC}$ transporters interaction and improved tumor uptake are required. Initial studies have commenced on one such second generation cyanoquinoline.

*AACR-Aflac, Incorporated, Scholar-in-Training Award winner.
Molecular imaging of the hedgehog signaling pathway in breast cancer. D.L. Smith ${ }^{1}$, R. Larson ${ }^{1}$, F. Kong ${ }^{1}$, D. Yang ${ }^{1}$, W. Woodward ${ }^{1}$, J. SimsMourtada $^{2}$; ${ }^{1}$ The University of Texas MD Anderson Cancer Center, Houston, TX, ${ }^{2}$ Helen F. Graham Cancer Center, Newark, DE

Introduction: The hedgehog $(\mathrm{Hh})$ signaling pathway plays a critical role in embryonic development and wound healing, and its aberrant activity is associated with several malignancies. Recent studies implicate Hh signaling in breast cancer growth and metastasis, and high tumor Sonic Hedgehog (SHh) expression is correlated with poor prognosis in invasive ductal carcinoma. SHh binds to the suppressive receptor Patched-1 (Ptch-1) and relieves the inhibition of the transmembrane protein Smoothened (Smo) by Ptch-1, resulting in the translocation of Gli transcription factors to the nucleus and activation of $\mathrm{Hh}$ target genes. In tumors with activated $\mathrm{Hh}$ signaling, Ptch-1 is overexpressed due to a feedback loop; therefore, we sought to image tumors with active Hh signaling by using radiolabeled peptides which bind the SHh docking site on Ptch-1. These molecular imaging probes have the potential to identify Hh-induced changes in Ptch-1 expression, which is useful for the imaging of aberrant Hh signaling in malignancies. Methods: For correlating Ptch-1 expression with the Ptch-1targeting peptide, the breast cancer cell line SKBR3 was co-stained with Ptch-1 and cultured with $100 \mathrm{nM}$ of the FITC-labeled peptide for four hours, after which cells were analyzed under a fluorescent microscope. To image Ptch-1 expression in vivo, peptides were conjugated with the chelator ethylenedicysteine (EC) and radiolabeled with ${ }^{99 \mathrm{~m}} \mathrm{Tc}$. Pilot studies were performed to examine the potential of imaging Ptch-1 receptor expression in a rat model of breast cancer. Fisher rats were inoculated with the mammary carcinoma cell line 13762, and after tumors grew for two weeks, rats were injected with approximately $250 \mu \mathrm{Ci}$ of the ${ }^{99 \mathrm{~m}} \mathrm{Tc}$-labeled peptide. Planar scintigraphy was conducted at 1,2, and 4 hours after injection of the radiolabeled peptide, and tumor-to-muscle ratios were calculated. After the first set of images were acquired, rats were treated with taxol and imaged again one week later. Results: The FITC-labeled peptide displays high correlation under a fluorescent microscope when co-stained with Ptch-1, suggesting specificity of the peptide targeting Ptch-1. In vivo imaging studies displayed greater accumulation of ${ }^{99 \mathrm{~m}} \mathrm{Tc}$-labeled peptides within tumors as compared to adjacent muscle tissue. Before treatment with taxol, tumor-to-muscle ratios were approximately 4 through all time points. After taxol treatment, the tumor-to-muscle ratio decreased slightly, albeit insignificantly. Significant peptide uptake was also observed in liver tissue, likely due to the conjugated FITC tag and endogenous Ptch-1 liver expression. Conclusions: These studies suggest that peptides which bind the SHh docking site in Ptch-1 correlate with Ptch-1 expression and can be used to image Ptch-1 in vivo. Further, our data suggests that radiolabeled peptides may enable us to examine the activity of the Hh signaling pathway and to evaluate response to anticancer therapies.

\section{0}

Melanin production by oncolytic vaccinia virus strains allows MR and optoacoustic deep-tissue imaging and laser-induced thermotherapy of cancer. J. Stritzker ${ }^{1}$, L. Kirscher ${ }^{2}$, M. Scadeng ${ }^{3}$, N.C. Deliolanis ${ }^{4}$, S. Morscher ${ }^{5}$, P. Symvoulidis ${ }^{4}$, K. Schaefer ${ }^{4}$, Q. Zhang ${ }^{1}$, M. Hess ${ }^{2}$, L. Buckel ${ }^{2}$, U. Donat ${ }^{2}$, W.G. Bradley ${ }^{3}$, V. Ntziachristos ${ }^{4}$, A.A. Szalay ${ }^{1}$; ${ }^{1}$ Genelux Corp., San Diego, CA, ${ }^{2}$ University of Würzburg, Würzburg, Germany, ${ }^{3}$ University of California San Diego, La Jolla, CA, ${ }^{4} \mathrm{Helmholtz}$ Zentrum München, Munich, Germany, ${ }^{5}$ iThera Medical GmbH, Munich, Germany

Here, the gene-evoked production of melanin as a new class of optical, optoacoustic and magnetic resonance imaging agent was tested using an oncolytic vaccinia virus platform in preclinical settings. Melanin was shown to be an excellent reporter for optical imaging without addition of substrate. In addition, melanin production resulted in strong signals in deep tissue optoacoustic imaging as well as magnetic resonance imaging. Moreover, melanin was used as theranostic agent, by using it as a target for laserinduced thermotherapy to enhance oncolytic viral therapy. In conclusion, melanin has several advantages over other reporters so far used in virotherapy and even in general. After ongoing extensive preclinical studies, 
oncolytic virus strains overproducing melanin might be used in clinical trials in cancer patients. Taken together, the melanogenic genes could replace other reporter genes especially in small animal studies and even in the clinic.

\section{1}

Evaluation of candidate near-infrared dyes for clinical translation of a tumor paint. S. Hansen ${ }^{1}$, M. Stroud ${ }^{1}$, J. Parrish-Novak ${ }^{1}$, C. Jochheim ${ }^{1}$, J. Olson'; ${ }^{1}$ Blaze Bioscience, Inc., Seattle, WA, ${ }^{2}$ Fred Hutchinson Cancer Research Center, Seattle, WA

Tumor Paints are being developed as real-time intraoperative guides for cancer surgeries. The first Tumor Paint being developed is a conjugate of a chlorotoxin (CTX) peptide and a near-infrared fluorophore. It specifically binds to cancer cells, causing the tumor tissue to fluoresce in the nearinfrared range while adjacent normal tissue is not fluorescent. Use of this Tumor Paint during resection has the potential to assist surgeons in obtaining negative tumor margins and sparing normal surrounding tissues. The broad tumor recognition of the CTX peptide justifies initial clinical trials in a variety of solid tumor types. Intraoperative imaging of the tumor, surrounding tissues, lymph nodes, and tumor bed following primary tumor excision would provide additional value in early clinical studies. Existing near-infrared intraoperative imaging devices are designed to detect indocyanine green (ICG), which is used in vascular surgeries, sentinel lymph node mapping, and perfusion studies. A variety of near-infrared dyes were conjugated to the targeting peptide, in order to produce a conjugate with excitation and emission spectra near those of ICG. ICG (Dojindo), IRDye800 (LiCor Biosciences), and DyLight-750 (Thermo Fisher Scientific) were conjugated to the peptide, and reaction products were analyzed by HPLC. The chlorotoxin based conjugates were tested in the ND2:SmoA1 mouse model of medulloblastoma. Each conjugate (20 nmole per mouse) was administered to 3 tumor-bearing and 3 wild type control mice via tail vein injection. Mice were imaged 1 day later using a Xenogen Spectrum imaging system. Mice were euthanized, the brain and all major organs were removed, frozen in OCT, sectioned, imaged using an Odyssey Near-Infrared imaging system (Li-Cor). All conjugates bound to tumor tissue and not areas of normal brain. The ratio of signal in tumor to that in normal brain from the same animal varied from 3.2-7.8. The ratio of signal in tumor of injected animals to that in wild type brain was 3.5-8.4 Fluorescence signals were detected using $765 \mathrm{~nm}$ excitation and 800-820 $\mathrm{nm}$ emission, settings consistent with those of imaging devices currently in clinical use. Use of conjugates fluorescing in these wavelengths would facilitate intraoperative imaging in early clinical trials.

\section{2}

Intrinsic indium-111 radiolabeled multidentate zwitterionic quantum dots for single-photon emission computed tomography and fluorescence imaging. M. Sun, G. Sundaresan, P. Jose, L. Yang, J. Zweit; Virginia Commonwealth University, Richmond, VA

Development of molecular or nano-scale probes combining multiple emission properties is fundamental for multimodal molecular imaging and cancer theranostics. Here we describe a strategy to incorporate indium-111 into near infrared (NIR) emission ultra-small Cu-In-Se (CIS) quantum dots (QDs) to make intrinsically radiolabeled nanoparticles for real time in vivo single-photon emission computed tomography (SPECT) and fluorescence imaging. To enhance stability and improve pharmacokinetics of $\mathrm{Cu}-\mathrm{In}(111)$-Se radiolabeled quantum dots (rQDs), a multidentate zwitterionic polymer ligand was synthesized and used to functionalize the CIS rQDs. Three-component micro-emulsion system was applied for successful ligand exchange of rQDs with multidentate zwitterionic polymer ligand. The stability of polymer ligand coated CIS rQDs was greatly enhanced in comparison with small zwitterionic rQDs. Reduced plasma/cell membrane nonspecific interaction of zwitterionic rQDs was observed. High passive uptake of zwitterionic rQDs $(\sim 0.5-0.6$ million rQDs/cell) was obtained in both COLO 205 and KB 3-1 cancer cell lines in 15 min determined by gamma counting, which could be due to direct cell membrane penetration of small zwitterionic nanoparticles. No obvious cytotoxicity was observed when COLO 205 and KB 3-1 cell lines were incubated with CIS rQDs for $24 \mathrm{hrs}$ (concentrations up to $75 \mathrm{nM}$ ). In vivo SPECT and fluorescence imaging of tumor bearing nude mice injected with CIS rQDs was acquired at different time points $(1 \mathrm{hr}, 4 \mathrm{hrs}$, $24 \mathrm{hrs}$ and $48 \mathrm{hrs}$ ). The SPECT signal corresponded well to fluorescence signal in all time points, which indicates high stability of multidentate zwitterionic rQDs in vivo. The pharmacokinetics of rQDs and their accumulation in tumor through enhanced permeability and retention (EPR) effect was monitored by SPECT/fluorescence imaging. Highest uptake of CIS rQDs in tumor at $4 \mathrm{hrs}$ post-injection was acquired and quantified by SPECT imaging. Multiplexed and sensitive fluorescence imaging combined with anatomical, quantitative SPECT/CT imaging demonstrated the advantage of multiple emissive CIS rQDs used for multimodal imaging. The intrinsically radio-labeling strategy provides a robust methodology to fabricate highly biological stable nanoprobes for long term in vivo biomedical imaging as well as reduce the interference of further surface modification compared with chelator radiolabel method. Feasible and reproducible synthetic procedures, low toxicity, high stability, tunable chemical/physical properties and improved physiological properties of multidentate zwitterionic intrinsic CIS rQDs make it a promising nanoprobe for multimodal molecular imaging and theranostic reagent for cancer diagnosis and therapy. Tumor targeting investigations are underway to enhance tumor accumulation and retention of these novel dual nano-probes.

\section{3}

Pegylated recombinant human hyaluronidase PH20 (PEGPH20) reduces ${ }^{18}$ FDG-PET uptake in mouse xenografts and phase 1 cancer patients. C. Thompson ${ }^{1}$, R. Korn ${ }^{2}$, X. Li ${ }^{1}$, J. Czernin 3 , C. Radu 3 , M. Borad ${ }^{4}$, A. Bessudo ${ }^{5}$, J. Zhu ${ }^{1}$, L. Wei ${ }^{3}$, D. Maneval ${ }^{1} ;{ }^{1}$ Halozyme, Inc., San Diego, CA, ${ }^{2}$ Imaging Endpoints, Scottsdale, AZ, ${ }^{3}$ University of California, Los Angeles, CA, ${ }^{4}$ Mayo Clinic, Scottsdale, AZ, ${ }^{5}$ California Cancer Associates for Research Excellence, Encinitas, CA

Pegylated recombinant human hyaluronidase PH20 (PEGPH20) is an investigational therapeutic agent under clinical development for use alone or in combination with other cancer therapies for the treatment of patients with non-hematologic malignancies that may accumulate hyaluronan (HA), a nonsulfated glycosaminoglycan that is a significant component of the extracellular matrix of many solid tumors. HA accumulation has been correlated with local invasion, the presence of distal metastasis, higher tumor grade, and poorer overall survival. Preclinical studies have demonstrated that sustained HA removal, accomplished with PEGPH20, inhibits tumor growth and enhances chemotherapeutic activity in HA-rich xenografts and genetically engineered mouse tumor models. As positron emission tomography (PET), following ${ }^{18}$ Fluorodeoxyglucose (FDG) administration, is a powerful modality for monitoring treatment response in solid cancers, we aimed to use ${ }^{18}$ FDG-PET imaging to evaluate the effects of PEGPH20 treatment on tumor metabolism. In brief, subcutaneous PC3 tumors were established in nude mice, and animals subsequently received a single intravenous dose of PEGPH20 $(4.5 \mathrm{mg} / \mathrm{kg})$ or vehicle. FDG was administered, and uptake was quantified in tumor tissue at $24 \mathrm{~h}, 72 \mathrm{~h}$ and $168 \mathrm{~h}$ post-dosing. Relative to the $24 \mathrm{~h}$ post dose time point, FDG tumor uptake $(\% \mathrm{ID} / \mathrm{g})$ in the PEGPH 20 treated cohort decreased by $15.6 \%$ at $72 \mathrm{~h}$ (from $9.6 \pm 2.1$ to $8.1 \pm 2.8$ ), and by $38.5 \%$ at $168 \mathrm{~h}$ (from $9.6 \pm 2.1$ to $5.9 \pm$ 2.1); whereas, in the vehicle treated group FDG uptake decreased by $9.2 \%$ at $72 \mathrm{~h}$ (from $7.9 \pm 0.73$ to $6.9 \pm 11$ ) and then remained unchanged at $168 \mathrm{~h}$ $(6.9 \pm 0.49)$. Concomitant to preclinical studies, ${ }^{18} \mathrm{FDG}$-PET imaging was conducted pre- and post-PEGPH20 treatment with 4 patients in a Phase 1 dose-escalation, safety, tolerability, PK/PD study of PEGPH20 treatment in patients with advanced solid tumors (NCT01170897). Patients presented with metabolically active tumors and were treated with $3.0 \mu \mathrm{g} / \mathrm{kg}$ PEGPH20 twice/week. Standardized uptake values (SUVs) were determined for multiple tumor lesions in all patients. Baseline SUVs were quantitatively compared to those measured at scheduled times up to 4 weeks after initiation of PEGPH20 treatment. Decreases in SUVs were noted in all patients, with maximum reductions in total SUVs exceeding $29 \%$ in each patient. Decreased SUVs ( $>30 \%$ baseline) in two patients were measured after 4-weeks of treatment, consistent with a partial metabolic response. Additional preclinical and clinical studies, complete with ${ }^{18}$ FDG-PET imaging, are ongoing to evaluate PEGPH20 for the treatment HA-rich solid tumors. 
approach due to associated toxicities may be successfully addressed with

Dissection of the role of the tumor microenvironment in oncogene addiction by ex vivo and in situ imaging. L. Tong, J. Jeon, B. Shen, R. Jianghong, F. Chin, S. Gambhir, D. Felsher; Stanford University, Stanford, CA

The targeted inactivation of oncogenes can elicit profound tumor regression through the mechanism of oncogene addiction. Previously, we have shown that oncogene addiction is associated with proliferative arrest, apoptosis, differentiation and senescence of tumor cells and the recruitment of immune effectors and the shutdown of angiogenesis in the host. Now, to dissect the contribution of the local tumor microenvironment to the mechanism of oncogene addiction we have used both ex vivo and in situ imaging approaches. We are using bioluminescence imaging, whole body fluorescent imaging, and microPET imaging to quantitatively measure the tumor growth and regression kinetics, apoptosis and proliferation changes upon oncogene inactivation with specific apoptosis and proliferation probes. We have developed an intravital microscopic imaging (IVM) strategy to monitor the dynamic and complex physiological processes and cell-cell interactions at a high resolution in the same tumor over time. We have designed imaging windows for IVM and molecular reporters to visualize multiple parameters during oncogene addiction including proliferation, apoptosis, senescence, angiogenesis, immune cell infiltration and trafficking. We have applied IVM to interrogate the spatiotemporal relationships between tumor cell-intrinsic and hostdependent changes and identify the specific role of immune effectors in regulating oncogene addiction. We have found that CD4+ $\mathrm{T}$ cells coordinated with other immune cells to regulate senescence, angiogenesis and tumor regression upon MYC inactivation in transgenic lymphoma mice. Through a novel PET-based approach we are able to monitor changes in proliferation and apoptosis in tumor cells upon oncogene inactivation. We have used these probes to perform a detailed analysis of the kinetics of changes in cellular proliferation and apoptosis. Moreover, through intravial imaging approaches to examine the time course of changes in the host immune response, we are able to measure changes in host immune cell homing to the site of the tumor before and after oncogene inactivation as well as changes in angiogenesis. Our results provide the first in situ examination of the mechanisms of oncogene addiction.

\section{5}

In vivo imaging of targeted antibody activation at the site of disease. O. Vasiljeva; CytomX Therapeutics Inc., South San Francisco, CA

Increased proteolysis is a hallmark of cancer; the progression, invasion and metastasis of tumors result from several interdependent processes in which proteases have been implicated. The increased proteolytic activity found in the vicinity of growing primary and metastatic tumors offers the opportunity for exquisitely specific targeting of tumor by using protease-activated antibody therapeutics, termed Probodies. Probodies combine the wellestablished power and specificity of monoclonal antibodies with a second level of tissue selectivity, driven by the action of endogenous disease enzymes. Probodies contain a masking peptide that blocks the antigenbinding site and is fused to the antibody through a substrate-containing linker. Through selection of a substrate linker that is cleaved by protease(s) preferentially localized or overexpressed in diseased tissue, but not in healthy tissues, a Probody can be constructed that is systemically stable, but cleaved locally at the site of disease. To monitor the protease activity required for Probody activation we developed, and validated in xenograft tumor models, a set of near infrared (NIR) quenched probes containing protease specific substrates. Moreover, the activation and binding of Probodies was monitored in vivo by use of fluorescently labeled Probodies. We have demonstrated efficacy of such molecules in xenograft tumor models and are actively pursuing preclinical development. Our data confirm that antibody activity can be more specifically targeted to diseased tissue by utilizing locally overexpressed proteases as activating agents, suggesting that a variety of antigens not previously amenable to an antibody therapeutic

*SNMMI/AACR Young Investigator Award winner. the Probody ${ }^{\mathrm{TM}}$ platform.

\section{6}

Molecular imaging reveals a role for AKT in resistance to cisplatin for ovarian endometrioid adenocarcinoma. H. Wang, S. Galbán, R. Wu, B. Bowman, A. Witte, K. Vetter, C.J. Galbán, B.D. Ross, K.R. Cho, A. Rehemtulla; The University of Michigan, Ann Arbor, MI

Purpose: Ovarian cancer is the fifth leading cause of cancer deaths among American women. Platinum-based chemotherapy, such as cisplatin, represents the standard of care for ovarian cancer. However, toxicity and acquired resistance to cisplatin have proven challenging in the treatment of ovarian cancer patients. Experimental Design: Using a genetically engineered mouse (GEM) model of ovarian endometrioid adenocarcinoma (OEA) in combination with molecular imaging technologies, we studied the activation of the AKT serine/threonine kinase in response to long-term cisplatin therapy. Results: Treatment of cells in culture and tumor-bearing animals with cisplatin resulted in activation of AKT, a key mediator of cell survival. Based on these results we investigated the therapeutic utility of AKT inhibition in combination with cisplatin, which resulted in enhanced and prolonged induction of apoptosis and in significantly improved tumor control compared to either agent alone. Conclusion: These results provide an impetus for clinical trials using combination therapy. To facilitate these trials, we also demonstrate the utility of diffusion-weighted MRI as an imaging biomarker for evaluation of therapeutic efficacy in OEA.

\section{$77^{\star}$}

Optical detection of viable tumor cells in liquid biopsies and their therapy in body fluids with oncolytic vaccinia virus. H. Wang ${ }^{1}$, N.G. $\mathrm{Chen}^{2}$, B. Minev ${ }^{2}$, M. Zimmermann ${ }^{3}$, M. Fells ${ }^{4}$, R. Aguilar ${ }^{4}$, Q. Zhang ${ }^{2}$, J. Sturm ${ }^{3}$, F. Feng ${ }^{3}$, U. Lauer ${ }^{3}$, A.A. Szalay ${ }^{1}$; ${ }^{1}$ University of Wuerzburg, Wuerzburg, Germany, ${ }^{2}$ University of California, San Diego, CA, ${ }^{3}$ Medical University Hospital, Tuebingen, Germany, ${ }^{4}$ Genelux Corporation, San Diego, CA

Nearly all cancer deaths result from the metastatic spread of cancer. Circulating tumor cells (CTCs), constituting each and every metastatic event, have been shown to be a useful surrogate marker for evaluating cancer prognosis and monitoring therapeutic response. Here, we report that viable migrating tumor cells including CTCs can be detected and eliminated by tumor-targeting oncolytic vaccinia virus (VACV). Tumor cell-specific recombinant VACVs that were derived from the LIVP strain and overexpress a bright far-red fluorescent protein TurboFP635 (Katushka) or GFP were used for imaging and enumeration of viable tumor cells, in an epithelial biomarker-independent manner, in body fluids. Using these virus strains, infected viable CTCs in the blood from mice bearing human prostate and lung cancers as well as in the blood from human patients with metastatic breast, colorectal, lung cancers, and melanoma were successfully detected and enumerated by optical imaging of the TurboFP635 or GFP protein. Similarly, viable tumor cells in the cerebrospinal fluid of a patient with latestage metastatic breast cancer were specifically detected. The VACV-based viable tumor cell infection approach compared favorably with the CellSearch ${ }^{\circledR}$ system regarding detection and enumeration of CTCs. In addition, CTCs identified by VACV were also shown to express cancer stem cell and epithelial-mesenchymal transition markers, exhibiting larger nuclei than the neighboring white blood cells under the microscope. Furthermore, we showed that treatment with a single intravenous injection of VACV resulted in elimination of CTCs in mice bearing late-stage human prostate cancer within two weeks. More importantly, a single intra-peritoneal delivery of VACV resulted in complete disappearance of cancer cells from the ascitic fluid of a gastric cancer patient with peritoneal carcinomatosis 8 days after treatment, in contrast to before-treatment ascites that contained $90 \%$ tumor cells. Taken together, VACV was shown to be a reliable tool for quantitative detection of viable tumor cells in liquid biopsies as well as a highly efficient therapeutic drug for treating viable tumor cells in body fluids from mice bearing human tumor xenografts and in ascitic fluids from human cancer patients. 
respectively, with $>95 \%$ purity. $\mathrm{C} / \mathrm{P}$ ratios were $2-3$ and did not significantly impact immunoreactivity. In vitro studies showed similar uptake profiles that were reduced by the presence of unlabeled agent. Tumors were clearly visualized by PET/CT with both radiotracers, although ${ }^{64} \mathrm{Cu}$-NODAGA-mAb7 showed slightly higher tumor/muscle ratios and lower liver signal. From the biodistribution data, we found similar tumor uptake $(>13 \% \mathrm{ID} / \mathrm{g})$ for both agents at 24 and $48 \mathrm{~h}$. Lower blood activity was observed with ${ }^{64} \mathrm{Cu}$-DOTA-mAb7, whereas ${ }^{64} \mathrm{Cu}-\mathrm{NODAGA}-\mathrm{mAb} 7$ had less accumulation in the liver and pancreas. Tumor/muscle ratios were comparable (15.9-17), while slightly higher tumor/blood ratios $(3.1 \pm 0.5$ vs. $1.9 \pm 0.6)$ were achieved with ${ }^{64} \mathrm{Cu}$-DOTAmAb7. Conclusions: To the best of our knowledge, this study represents the first comparison of ${ }^{64} \mathrm{Cu}$-labeled DOTA and NODAGA immunoconjugates in vivo. Our results showed that the use of NODAGA did not significantly change targeting properties of the EpCAM specific antibody when compared to DOTA. The blood activity of each radiotracer decreased from 1-48 h suggesting minimal transchelation of ${ }^{64} \mathrm{Cu}$ and comparable in vivo stability. Tumor uptake observed in imaging and biodistribution studies indicates the utility of both chelation schemes for agent development. However, the ability to form ${ }^{64} \mathrm{Cu}$-NODAGA complexes at room temperature could be advantageous for heat-labile compounds, such as engineered antibody fragments, that have excellent potential for molecular imaging but may degrade during radiolabeling procedures that require elevated temperatures.

effusion. In GLV-1h68-treated tumor-bearing mice, ME was highly attenuated compared to untreated mice, judged by MRI. Virus-mediated reduction of tumor cell-derived VEGF levels in tumors, decreased invasion of tumor cells into the peritumoral tissue, and viral infection of blood vessel-invading tumor cells were contributing to this process. Moreover, we showed that the use of oncolytic VACV encoding for a single-chain antibody against VEGF (GLV-1h108) significantly enhanced mono-therapy of the oncolytic agent. Fluorescence-imaging of PC14PE6-RFP tumor growth (RFP) and GLV-1h68 or GLV-1h108 infection (GFP) revealed that in GLV1h68-infected animals both the RFP and the GFP signal increased during the 21-day observation period. However, the RFP signal outgrew the GFP signal at later time points indicating that the viral infection progressed more slowly than tumor growth per se. In contrast, in GLV-1h108-injected animals both the RFP and the GFP signal simultaneously declined indicating the progress of virus-dependent tumor growth elimination. Taken together, the presented results suggest oncolytic virotherapy using tumor-specific VACV encoding for a single-chain antibody against VEGF to be a promising treatment option of ME in the future with the potential to improve the currently palliative care of patients.

\section{9}

Comparison of ${ }^{64} \mathrm{Cu}$-labeled DOTA- and NODAGA-immunoconjugates that target epithelial cell adhesion molecule in prostate cancer. $\mathrm{N}$. Wilganowski, B.R. Harvey, K. Pinkston, H. Robinson, K. Gore, G. Dickinson, S. Ghosh, E. Sevick-Muraca, A. Azhdarinia; University of Texas Health Science Center Houston, Houston, TX

Objectives: Bifunctional chelators have been shown to impact the biodistribution of antibody-based imaging agents. Recently, radiolabeled NODAGA-peptide complexes have demonstrated improved in vivo stability compared to DOTA counterparts, encouraging similar characterization with antibody-based agents. Here, we developed and compared ${ }^{64} \mathrm{Cu}$-labeled NODAGA- and DOTA-antibody conjugates for the detection of epithelial cell adhesion molecule (EpCAM) in a prostate cancer model. Methods: DOTA- and NODAGA-conjugates of an EpCAM targeting antibody (mAb7) were synthesized using standard NHS chemistry and purified by size exclusion. Biological activity of the resulting immunoconjugates was determined by flow cytometry and ELISA assays. The agents were radiolabeled with ${ }^{64} \mathrm{Cu}$ in $0.1 \mathrm{~N}$ sodium acetate (DOTA: $40^{\circ} \mathrm{C}$ for $1 \mathrm{~h}$; NODAGA: $25^{\circ} \mathrm{C}$ for $1 \mathrm{~h}$ ), purified by size exclusion, and analyzed by radio-TLC and radio-HPLC. Chelator/protein $(\mathrm{C} / \mathrm{P})$ ratios were quantified using non-radioactive $\mathrm{Cu}$ as a carrier. $\mathrm{PC} 3$ prostate cancer cells expressing the DsRed gene reporter were used to characterize receptor binding in vitro and to establish a xenograft tumor model in nude mice. PET/CT imaging and tissue biodistribution studies were performed to assess tumor targeting, non-target uptake, pharmacokinetics and stability. Results: ${ }^{64} \mathrm{Cu}-\mathrm{NODAGA}-\mathrm{mAb} 7$ and ${ }^{64} \mathrm{Cu}$-DOTA-mAb7 were obtained in radiochemical yields of 60 and $70 \%$,

80

Patient-specific model of NaF PET to facilitate multicenter clinical trial PET imaging protocols and analysis: preliminary results of a CT-based simulation. J.M. Wilson, K.L. Chisholm, J.D. Oldan, W.P. Segars, T.G. Turkington, B.B. Chin; Duke University Medical Center, Durham, NC

Purpose: ${ }^{18} \mathrm{~F}$ sodium fluoride $(\mathrm{NaF}) \mathrm{PET} / \mathrm{CT}$ is a highly sensitive, accurate, and clinically available method to identify osseous metastases, particularly in low FDG avidity tumors such as prostate cancer. Multicenter clinical trials using NaF PET to assess the efficacy of novel therapies for bone metastases could be greatly facilitated by standardized acquisition and processing protocols that can reduce variability. A patient-specific model of $\mathrm{NaF}$ PET is being developed that simulates NaF PET images from CT, allowing PET image quality to be estimated as a function of injected dose, uptake time, and scan technique. This patient-specific model of normal uptake, when compared with actual NaF PET uptake, can identify abnormalities. This facilitates the development of a semi-automated quantification of disease burden. Procedures: All anonymized NaF PET/CT datasets $(n=4)$ were obtained after IRB approval. The osseous structures were isolated by CT segmentation that included the following steps: 1) masking out non-anatomic structures including the patient bed, 2) applying median filtering, 3) automated region dilation and erosion, and 4) manual correction of osseous contours. Tissue compartments were assigned by segmentation of adipose (FAT), soft tissue (ST) and air (A) by Hounsfield units (HU); trabecular bone (TB) and cortical bone (CB) were further subclassified based on HU. After segmentation, normal NaF PET radioactivity was simulated by assigning uptake values to the above compartments based on literature estimates of bone turnover (FAT:ST:CB:TB :: 0.5:1:1-10:10-30) and observed normal uptake in actual normal $\mathrm{NaF}$ scans. Simulated data was further processed by spatially blurring to simulate PET resolution, forward projecting, and attenuating by the CT to obtain sinogram data. Sinograms were scaled by a factor that represented the scanner sensitivity, resulting in realistic count levels. At this sinogram level, Poisson noise (at several levels) was then added to simulate detected counts of several scan durations. The simulated PET sinogram was then reconstructed with attenuation correction. To test the method, simulated $\mathrm{NaF}$ data were visually compared to actual $\mathrm{NaF}$ data from the same patients by image subtraction after normalization. With a working patient-specific CT-based simulation of $\mathrm{NaF}$ images, we can study the impact that injected dose, uptake time, scan time, and other key parameters have on final image quality, including variability. This simulation platform of normal NaF uptake can also be used in comparison with the true acquired $\mathrm{NaF}$ data to identify abnormalities, and serve as the foundation of a semi-automated quantification technique of disease burden. Data: Using the determined single scaling factor, the simulated to actual $\mathrm{NaF}$ count ratios were 0.944 , 
$1.01,1.10$, and 0.96 , where 1.0 represents the same number of acquired and simulated counts. For the above dataset $(n=4)$, several Poisson noise levels were introduced into the simulated PET sinograms, resulting in realistic high to low noise images. For the four datasets, $[6,7,1,5]$ abnormalities were counted by visual inspection on the acquired data, and $[6,6,1,5]$ abnormalities were counted on the subtraction image sets. Conclusions: This platform produces simulated normal NaF PET images that are realistic in appearance and reveal plausible lesions when subtracted from actual $\mathrm{NaF}$ images. Such a framework allows parameterizations of the physical parameters (spatial resolution, noise, imaging time), and can model physiologic effects (differences in tumor size, tumor location, levels of contrast, radiotracer uptake, background clearance) to estimate quantitative effects on the final reconstructed PET image. Finally, mismatches between the ideal simulated normal NaF PET image and the actual acquired PET image can be used to identify abnormalities and facilitate a semi-automated quantification of disease burden. Future work will be to introduce artificial tumors of a range of sizes and uptake ratios into the simulation model to evaluate requisite levels for detectability. Analysis algorithms will count and quantify disease burden, and will be compared with current clinical methodologies.

\section{1}

Noninvasive imaging of tumor glycogen storage by ${ }^{18}$ F-NFTG positron emission tomography. T.H. Witney, L.S. Carroll, I.S. Alam, R. Sala, R. Agarwal, E.O. Aboagye; Imperial College London, London, United Kingdom

The majority of tumours exhibit substantially increased levels of glucose uptake and utilisation in comparison to non-neoplastic tissues. As well as increased glycolytic flux, tumour cells have also been shown to accumulate glycogen at levels far higher than their normal corresponding tissues. Interest in the role of glycogen storage in malignant cells has arisen following the discovery that increased expression of the small GTPase, $R a b 25$, in ovarian adenocarcinoma resulted in significantly increased glycogen storage, correlating to increased tumour aggressiveness and resistance to therapy-induced apoptosis. Development of a novel noninvasive measure of tumour glycogen would therefore be highly advantageous. It has been shown previously that the fluorescent glucosamine analogue, 2-NBDG, can be used to assess glycogen storage in vitro. In this study we show that tumour glycogen storage can be imaged in vivo by positron emission tomography with ${ }^{18} \mathrm{~F}-\mathrm{N}$-(methyl(2fluoroethyl)-1H-[1,2,3]triazole-4-yl)glucosamine $\left({ }^{18} \mathrm{~F}-\mathrm{NFTG}\right)$. Temporal uptake of ${ }^{18} \mathrm{~F}$-NFTG was compared in isogenic HEY pcDNA and HEY Rab25 cells over a 4-h time course. A significant increase in ${ }^{18} \mathrm{~F}-\mathrm{NFTG}$ was observed in Rab25-transfected cells in comparison to empty vector controls at $4 \mathrm{~h}$, with uptake of $3.2 \pm 0.25 \%$ radioactivity $/ \mathrm{mg}$ protein and $2.4 \pm 0.17 \%$ radioactivity $/ \mathrm{mg}$ protein, respectively. Increased uptake in HEY Rab25 paralleled elevated punctate 2-NBDG intracellular localization, shown by fluorescence microscopy $(500 \mu \mathrm{M} ; 3 \mathrm{~h})$, indicative of glycogen labeling. It has been shown previously that glycogen storage rapidly increases at the beginning of the stationary growth phase in cultured colorectal adenocarcinoma cells. Here we showed that total glycogen, 2-NBDG fluorescence and ${ }^{18} \mathrm{~F}$-NFTG uptake increased with increasing HEY Rab25 cell density, whereas ${ }^{18}$ F-FDG uptake did not change when cells reached stationary phase. In vivo imaging of implanted HEY Rab25 xenografts showed rapid tumor labeling, followed by slow wash-out of the tracer. Unlike FDG, liver-specific retention was observed, with low brain uptake. In a comparative study with FDG, muscle uptake was elevated 1.5 -fold with ${ }^{18} \mathrm{~F}-\mathrm{NFTG}$. In a further study, IGROV-1 ovarian carcinoma cells, containing a heterozygous PTEN mutation, were found to display a 4.8-fold increase in total glycogen in vitro when compared to HEY Rab25, corresponding to a 4.3 -fold increase in ${ }^{18} \mathrm{~F}-\mathrm{NFTG}$ uptake. This increase in ${ }^{18} \mathrm{~F}$-NFTG uptake translated to a doubling of tumor uptake in vivo, measured from the area under the time vs. radioactivity curve. We have demonstrated here a novel method for the non-invasive imaging of tumor glycogen, both in cells and in vivo. Given the correlation between glycogen storage and tumor aggressiveness, the use of ${ }^{18} \mathrm{~F}-\mathrm{NFTG}$ may provide complementary information to FDG. Enhanced ${ }^{18} \mathrm{~F}-\mathrm{NFTG}$ uptake by quiescent cells may have clinical relevance - for detection of

*AACR-Aflac, Incorporated, Scholar-in-Training Award winner. residual disease and predicting efficacy of drugs that target only cycling cells - and warrants further evaluation.

\section{2}

Noninvasive imaging of tumor apoptosis in NSCLC with ${ }^{18}$ F-ICMT-11 positron emission tomography. T.H. Witney, A. Chandrashekran, R. Fortt, E.O. Aboagye; Imperial College London, London, United Kingdom

Tumor response to therapy is assessed primarily in the clinic by monitoring reductions in tumor size. However, this approach lacks sensitivity since in many cases several weeks may elapse before there is evidence of tumor shrinkage. There is therefore a need to develop noninvasive imaging techniques for monitoring tumor treatment response in the clinic. It has been shown recently that the uptake of ${ }^{18} \mathrm{~F}$-ICMT- 11 can be used to detect response to therapy by positron emission tomography (PET) in murine lymphoma. The aim of the present study was to determine whether ${ }^{18} \mathrm{~F}$-ICMT-11-PET could be used to detect response to therapy in two models of non-small cell lung cancer (NSCLC) with varying sensitivities to carboplatin. Carboplatin treatment resulted in a linear dose-dependent increase in caspase-3/7 activation in sensitive PC-9 cells, confirmed by measurements of caspase-3-mediated polyADP-ribose polymerase cleavage. In the resistant A549 cells, apoptosis was not induced up to $200 \mu \mathrm{M}$ carboplatin. Carboplatin treatment induced a proportional increase in ${ }^{18} \mathrm{~F}$-ICMT-11 uptake in PC-9 cells in vitro; a nine-fold increase in uptake was measured with $200 \mu \mathrm{M}$ carboplatin in comparison to vehicle treated cells $(\mathrm{P}<0.01)$. No changes in uptake were observed in A549 cells following carboplatin treatment. ${ }^{18} \mathrm{~F}$-ICMT-11 uptake was shown to correlate well with caspase-3/7 activity, whose activation is pivotal for the execution of the apoptotic programme. These data show that ${ }^{18} \mathrm{~F}-\mathrm{ICMT}-11$ can probe tumour apoptosis and has the potential to differentiate between responders and non-responders in NSCLC following therapy.

\section{$83^{\star}$}

Analyzing the response to metabolic therapy in a mouse model of malignant glioma using multimodality imaging. E.C. Woolf, G. Turner, M.C. Preul, Q. Liu, A.C. Scheck; Barrow Neurological Institute, Phoenix, AZ

The ketogenic diet $(\mathrm{KD})$ is a therapeutic high-fat, low-carbohydrate diet that alters metabolism by increasing the level of ketone bodies in the blood. We have used a bioluminescent intracranial mouse model of malignant glioma and the IVIS ${ }^{\circledR}$ Spectrum in vivo imaging system (Caliper Life Sciences, a PerkinElmer Co, Hopkinton, MA) to demonstrate that a rodent ketogenic diet given ad libitum increases blood ketones without altering animal weight or overall blood glucose levels. Animals maintained on a KD have extended survival following tumor implantation, and the KD potentiates the therapeutic effects of radiation and chemotherapy. We previously demonstrated in vivo that these tumors cause a local increase in reactive oxygen species (ROS), but the KD reduced ROS in the tumor when quantitatively analyzed ex vivo. We now hypothesize that ketosis, which is known to have a neuroprotective effect in various diseases, may also reduce peritumoral edema and/or inflammation. Cyclooxygenase $2(\mathrm{COX}-2)$ is a known mediator of inflammation, and expression profiling analysis showed that animals maintained on a SD have a significant increase in expression of the gene encoding COX-2 in their tumor, while animals maintained on KD showed no such increase. MRI analyses using a Bruker Biospec 70/30 MRI (Bruker Daltonics Inc., Freemont, CA) showed that animals fed KD also have a significant reduction in peritumoral edema. We previously demonstrated that commercially available fluorescent probes such as AngioSense 680 EX (PerkinElmer, Inc.) can be used in concert with in vivo imaging of tumor bioluminescence to demonstrate vascular permeability that can lead to increased peritumoral edema. We are now using the XenoLight ${ }^{\circledR}$ RediJect COX-2 fluorescent probe (PerkinElmer, Inc) and the AngioSense 680 EX fluorescent probe in combination with the bioluminescent indication of tumor size and MRI analyses of tumor size and peritumoral edema. Co-registration of these images will be done using a mouse imaging shuttle and multi-modality software module (Caliper Life Sciences), thus facilitating in vivo analysis of the mechanisms of action of the $\mathrm{KD}$ on tumor gene expression and growth. 
Endosomalytic anionic polymer for the cytoplasmic delivery of siRNA in localized in vivo applications. B. $\mathrm{Xu}^{1}, \mathrm{~S}$. Khormaee ${ }^{2}, \mathrm{Y}$. $\mathrm{Choi}^{2}, \mathrm{M}$. $\mathrm{Shen}^{2}$, H. Wu ${ }^{1}$, G. Griffiths ${ }^{3}$, R. Chen ${ }^{4}$, N. Slater ${ }^{4}$, J. Park ${ }^{2}$; ${ }^{1}$ IPDC/NHLBI/ $\mathrm{NIH}$, Rockville, MD, ${ }^{2} \mathrm{NINDS} / \mathrm{NIH}$, Bethesda, MD, ${ }^{3} \mathrm{MIP} / \mathrm{NCI} / \mathrm{NIH}$, Bethesda, MD, ${ }^{4}$ University of Cambridge, Cambridge, United Kingdom

Introduction: RNA interference (RNAi) has emerged as an important too for gene therapy. RNAi directed against the chemoresistance protein stathmin reduces its level and increases the sensitivity of malignant glioma cells to specific types of chemotherapeutic agents, holding promise for improved malignant glioma treatments. However, for small interfering RNA (siRNA) effectiveness depends on escape from endosomal compartments once internalized. Furthermore, dissociation of siRNA from polymer carriers in cytoplasm is a critical step for efficient siRNA delivery. At present, efficient delivery of siRNAs remains a major barrier to their clinical application. In this study, a novel polymer, phenylalanine-grafted poly(L-lysine iso-phthalamide) (PPLI) was examined for its ability to deliver siRNA to glioma cells. Methods: Conjugation of PPLI with Alexa Fluor 647 was performed to examine the mechanism of PPLI internalization prior to undertaking siRNA delivery. For delivery of siRNA, we introduced a disulfide bond between carrier PPLI and siRNA to allow for effective dissociation of siRNA from PPLI in the reductive conditions of the cytoplasm. Results: PPLI is effective at mediating endosomal disruption in over $95 \%$ of cells studied. For delivery of siRNA, A PPLI-siRNA conjugate against stathmin silences its expression both in vitro and in vivo Furthermore, our results indicate that the conjugate is highly effective at inhibiting tumor growth when combined with the nitrosourea chemotherapy agent carmustine in a subcutaneous malignant glioma model. Conclusions: PPLI is effective at mediating endosomal pore formation which presents as a promising new vehicle for the safe and efficient delivery of siRNAs and potentially other nucleic acids for therapeutic purposes.

\section{5}

An improved noninvasive imaging method for the assessment of changes in tumor microstructure using temporal diffusion spectroscopy MRI. J. Xu, D.C. Colvin, K. Li, R.A. Smith, H.C. Manning, J.C. Gore; Vanderbilt University, Nashville, TN

A novel magnetic resonance imaging method for the assessment of tissue microstructure has been developed, and its potential role as a quantitative imaging biomarker has been evaluated in studies of tumors and their responses to treatments. The method provides information on the various scales that characterize cellular structures that restrict the free movement of water within tissues, and in a proliferating tumor can report on changes within cells that occur prior to gross changes in cell density. Diffusionweighted magnetic resonance imaging (DW-MRI) is currently in widespread use as a non-invasive, non-radioactive approach to measure tumor cellularity, and therefore has been suggested as a promising imaging biomarker for characterizing tumors and assessing their therapeutic responses to cancer treatments. Conventional DW-MRI reports primarily on changes in overall cell density, which usually decreases when tumors are successfully treated. However, in general, tumors may show increased or decreased cellularity depending on the type of cancer, its state of development and the treatment type, so single measures using DW-MRI may be misleading. We have developed an improved DW-MRI method that uses diffusion gradients oscillating at different frequencies in place of conventional static gradients, which we term temporal diffusion spectroscopy (TDS). Such a method is capable of separating the influence of small-scale structures from variations in gross cell density. TDS is in principle able to distinguish different microstructural variations in tissues from subcellular to supracellular levels. To demonstrate the capabilities of TDS and its potential role in imaging tumors, we have performed (1) elaborate computer simulations of diffusion in arrays of cells of various complexities, which suggest that changes in cell nuclear size are detectable; (2) in vitro cell studies showing the effects of various drugs and cell synchrony on temporal diffusion spectra; (3) in vivo imaging studies in a rat glioma model, showing that the new approach can reveal greater detail and heterogeneity within tumors than conventional methods, and (4) preliminary studies of early treatment response in mouse SW620 (a human colon cancer) tumor xenografts treated by barasertib, a selective inhibitor of Aurora B kinase. These confirm that diffusion measurements at different length scales are crucial for the full interpretation of diffusion changes, and suggesting that intracellular changes that occur early in response may be detected before there are gross changes in cell density. In continuing studies, the response of different tumor types to different treatment regimens is being more comprehensively evaluated, and histological and immunohistochemical data are being acquired for validation and correlation with in vivo imaging results. Temporal diffusion spectroscopy is readily implemented on small animal MRI systems and appears to provide novel information for high resolution imaging assessments of tumor status and for monitoring tumor early response to treatment non-invasively.

\section{INVITED SPEAKER ABSTRACTS}

\section{1}

Location, location, location-visualizing interactions between Helicobacter pylori and the epithelium of the gastric glands. M.R. Amieva; Stanford University School of Medicine, Stanford, CA

Chronic infection of the stomach by Helicobacter pylori is the strongest known risk factor for the development of gastric cancer. The more virulent strains of $\mathrm{H}$. pylori adhere to the gastric epithelium and use a macromolecular syringe, a type four secretion system, to inject the virulence factor CagA into the epithelial cells. Cell culture experiments show that CagA acts as a signaling molecule inside host cells. It alters cell polarity and perturbs the cell junctions, activates growth factor signaling, inhibits apoptosis, and can promote tumor development. However it is unknown when and to which cells CagA is delivered in vivo. We used several imaging approaches to study how bacteria associate with the epithelium and their role in disease. Using time-lapse microscopy of infected polarized cells in culture we found that adhered bacteria can replicate directly on the epithelial junctions forming microcolonies on the cell surface. CagA aides the bacteria to survive in this niche by altering cell polarity and allowing micronutrients to reach the bacteria. To study this process in vivo, we developed a murine model of gastric infection with an $\mathrm{H}$. pylori strain that delivers CagA. Over a period of 3-6 months infected mice develop metaplastic lesions in the stomach mucosa. To find where $\mathrm{H}$. pylori interact with the gastric epithelium we reconstructed these infected murine stomachs in 3D using confocal microscopy at high resolution. We discovered two distinct populations of infecting bacteria. One population is found free-swimming in the surface mucus and occasionally adhered to the entrance of the glands. A second and distinct population is found deep in the gastric glands. These H. pylori form microcolonies directly on the epithelial junctions of cells in the mid-zone of the glands. This region of the glands is populated by gastric epithelial precursor cells that are actively dividing, and $\mathrm{H}$. pylori directly colonizes proliferating host cells. Bacterial mutants that can colonize the surface mucus but cannot form microcolonies in the glands fail to cause pathology. We hypothesize that $\mathrm{H}$. pylori use the gastric precursor cell niche as a reservoir site of bacterial colonization, and that its effects on this cell population contribute to neoplastic progression.

\section{2}

Imaging the tumor microenvironment: an ever-changing landscape. Z.M. Bhujwalla; Johns Hopkins University School of Medicine, Baltimore, MD

The networks of blood and lymphatic vessels, stromal cells, and the extracellular matrix (ECM) that are collectively termed the tumor microenvironment (TME) are frequently co-opted and shaped by cancer cells to survive, invade and form distant metastasis. Abnormalities in vasculature, lymphatics, and metabolism impose additional heterogeneities in hypoxia, interstitial pressure and acidic microenvironments. The TME therefore presents an ever-changing landscape of spatial and temporal heterogeneities in physiology, metabolism and stromal cell trafficking. With an enviable capacity to adapt and survive continually changing environments, cancer represents the epitome of a successful organization. Many of the lethal consequences of these changing landscapes such as hypoxia create a cascade of changes in multiple pathways and networks, some of which may have lethal repercussions such as recurrence and 
metastasis that are only evident several years later. Multi-modal molecular and functional imaging fill important niches in the dynamic visualization of many characteristics of the TME such as the extracellular matrix (ECM), cancer associated fibroblasts (CAFs), adipocytes, pericytes, multiple immune cells such as tumor associated macrophages (TAMs), and vascular and lymphatic endothelial cells. Integrated advances incorporating imaging with molecular biology and chemistry are providing unique capabilities to understand the role of the TME in several aspects of tumor growth and treatment, and to image the interactions between cancer cells and stromal cells. Several aspects of the TME can be mined for targets to exploit for treatment and for the purpose of theranostic imaging where noninvasive imaging based detection of a target is combined with the delivery of a therapeutic payload to that target. Image-guided delivery of multiple siRNA selectively to a tumor, visualization of this delivery via liposome or nanocarrier technology, and detection of a response are now well within current imaging capabilities. Targeting specific microenvironments or stromal compartments with theranostic agents, imaging cancer stem cells, and imaging and targeting permissive or preventive microenvironmental niches for cancer stem cells are areas that are likely to have significant impact on cancer research and treatment. A major challenge is to identify primary tumors that are likely to metastasize. For this to be achieved, imaging biomarkers of specific microenvironments and molecular pathways that identify tumors with a predilection for metastasis will have to be identified. This would allow image-guided targeting of specific microenvironments that are most likely to permit metastatic dissemination.

\section{3}

Imaging abnormal metabolism in cancer: association with an altered tumor microenvironment, immunological dysfunction and a propensity to develop metastases. R. Blasberg ${ }^{1,2,3}, \mathrm{R}$. Khanin ${ }^{* 4}$, J. Koutcher*3,5,6,7; ${ }^{1}$ Department of Neurology, Memorial Sloan-Kettering Cancer Center, New York, NY, ${ }^{2}$ Department of Radiology, Memorial Sloan-Kettering Cancer Center, New York, NY, ${ }^{3}$ Molecular Pharmacology and Chemistry Program, Memorial Sloan-Kettering Cancer Center, New York, NY, ${ }^{4}$ Bioinformatics Core, Memorial Sloan-Kettering Cancer Center, New York, NY, ${ }^{5}$ Department of Medical Physics, Memorial Sloan-Kettering Cancer Center, New York, NY, ${ }^{6}$ Department of Medicine, Memorial Sloan-Kettering Cancer Center, New York, NY, ${ }^{7}$ Department of Physiology and Biophysics, Weill Cornell Graduate School of Medical Sciences, New York, NY

Abnormal glucose, pyruvate/lactate and glutamine metabolism in tumors can be studied optimally by multi-modality imaging, including multinuclear MRS/MRSI studies ${ }^{1} \mathrm{H},{ }^{13} \mathrm{C},{ }^{31} \mathrm{P}$ etc), hyperpolarized-MRS/MRSI, and by ${ }^{11} \mathrm{C} /{ }^{18} \mathrm{~F}$ PET). These assessments can be used to characterize an abnormal tumor microenvironment that is associated with an altered immune response ( $\mathrm{T}$ cell dysfunction) and a propensity to develop metastases in human tumors. Metabolic changes occur in solid tumors as the disease evolvesduring tumor initiation, growth, progression and the development of metastases. It has been shown that tumors with high glucose utilization, high tissue lactate concentrations and high expression of lactate dehydrogenase A (LDH-A) have been linked to poor prognosis, and are associated with a greater propensity to develop metastases. We recently showed that high expression of LDH-A, LDH-B and monocarboxylate transporters (MCTs) is associated with a propensity to develop metastases and poor outcome in an analysis of 4 clinical data sets (671 breast cancer patients). It has also been shown recently that changes in tumor metabolism and the tumor microenvironment can have a profound effect on $\mathrm{T}$ cell tumor-infiltration and function. The ability of tumors to evade effective immune responses remains a major impediment to successful treatment of most malignancies. The development and testing of novel strategies to overcome this impediment is a very active area of current research. Much of this research focuses on inhibitory molecules, cytokines and the infiltration of immune inhibitory cells. However, we are taking a somewhat different approach by focusing on the effects of abnormal tumor metabolism on $\mathrm{T}$ cells; specifically the effects of extracellular $\mathrm{pH}(\mathrm{pHe})$ and lactate in the tumor microenvironment. It is well known that $\mathrm{T}$ cells rapidly transition from a mitochondrial-dominant oxidative phosphorylation phenotype to an aerobic glycolytic phenotype following activation, resulting in an $\sim 20$-fold increase in glucose utilization and an $\sim 40$-fold increase in lactate production. New strategies for modulating the tumor microenvironment, including $\mathrm{pHe}$ and lactate, are being developed and some are being translated to the clinic using specific drugs. We expect that "normalization" of the tumor microenvironment (pHe and lactate, and possibly hypoxia) will significantly enhance adoptive $\mathrm{T}$ cell therapy. Furthermore, the recent associations between specific genetic alterations and specific metabolic changes in cancer cells have stimulated renewed interest in the role of cancer cell metabolism. Lactate accumulation in tumor cells is associated with aerobic glycolysis (Warburg effect), and metabolic changes in primary tumors have a significant impact on the development of the metastatic phenotype. We have focused on several components of cell metabolism that have significant effects on the tumor microenvironment. LDH-A is a bridge between anaerobic and aerobic glycolysis, as well as glutamine metabolism, whereas CAIX and MCT4 are primarily involved in the export of lactate from cancer cells and acidification of the extracellular space. Hyperpolarized ${ }^{13} \mathrm{C}$-MRS, multinuclear MRS and PET have unique capabilities that are complementary when used together. PET is exquisitely sensitive, capable of measuring concentrations in the picomolar range, and is quantitative in absolute terms. However, PET cannot directly determine the identity of the molecule bearing the radiolabel, whereas hyperpolarized ${ }^{13} \mathrm{C}$-MRS is able to distinguish multiple metabolites. Combining PET with hyperpolarized ${ }^{13} \mathrm{C}$ MRS of the same compound, for example, $\left[1-{ }^{11} \mathrm{C}\right]$-pyruvate and $\left[1-{ }^{13} \mathrm{C}\right]$-pyruvate, can overcome this deficiency and improve the accuracy of the PET pharmacokinetic model parameters. While the combination of ${ }^{11} \mathrm{C}$ PET and hyperpolarized ${ }^{13} \mathrm{C}$-MRS promises to improve the accuracy and robustness of measurements of enzyme and transporter activity in vivo, neither is able to measure the concentration of endogenous pools. ${ }^{1} \mathrm{H}-\mathrm{MRS}$ (e.g., lactate), however, is capable of making these measurements. Thus, the combination of all three modalities is most robust. For example, this combination will allow us to determine in vivo whether a given chemotherapeutic agent targeting LDH-A is successful in reducing its metabolic activity. Also, we will be able to ascertain whether the changes in LDH-A activity effect changes in the lactate levels, the pyruvate/lactate metabolites and $\mathrm{pHe} .{ }^{*}$ Coauthors.

\section{4}

Cerenkov luminescence imaging: a new tool for molecular imaging? S. Cherry; University of California, Davis, CA

Cerenkov radiation is a phenomenon where optical photons are emitted when a charged particle moves through a dielectric medium faster than the speed of light in that same medium. Recently, a number of laboratories have shown that measurable visible light due to the Cerenkov effect is produced in vivo following administration of $\beta$-emitting radionuclides to small animals. Furthermore, the amounts of injected activity required to produce a detectable signal are consistent with small animal molecular imaging applications. This observation has led to the development of a new hybrid molecular imaging modality known as Cerenkov luminescence imaging (CLI) that allows the spatial distribution of biomolecules labeled with $\beta$-emitting radionuclides to be imaged in vivo using sensitive chargecoupled device (CCD) cameras. CLI allows many common radiotracers to be imaged on widely available in vivo optical imaging platforms and, more importantly, provides a pathway for directly imaging $\beta$-emitting radionuclides that are being developed for therapeutic applications in cancer and that are not readily imaged by existing methods. This presentation will review the physics of Cerenkov radiation as it relates to in vivo molecular imaging and present computational results that predict the light yield and spatial distribution for a range of biomedically-relevant radionuclides. In vivo studies in mouse models using both $\beta^{+}$and $\beta$-emitting radionuclides will be shown in order to illustrate possible applications of the technique. Methods to spectrally shift the Cerenkov light also will be described. Finally, the potential for clinical applications will be discussed, and the strengths and weaknesses of Cerenkov imaging compared with other nuclear imaging techniques defined.

Imaging analysis of targeted adenoviral vectors for rational design advancement and for clinical monitoring. D.T. Curiel; Washington University School of Medicine, St. Louis, MO

Adenoviral vectors (Ad) have been increasingly employed for cancer therapy applications in the context of gene therapy, virotherapy and vaccine 
approaches. For each of these applications, it has been recognized that modifications of the virus tropism to accomplish target cell specific delivery can accrue direct therapeutic index gains. On this basis, a range of vector modification strategies has been endeavored to alter adenoviral tropism towards this goal. In this regard, imaging analysis has allowed direct determination of vector gains in the context of in vitro and in vivo model systems. Such assessment requires assay of the modified distributions of both vector particles and delivered transgenes. For the former aspect, we have employed a strategy of direct incorporation of fluorescent markers into the viral capsid via genetic methods. For the latter assay, we exploit a range of imaging reporters expressed as vector genome-incorporated transgenes for non-invasive monitoring. Translation of these various approaches into the human clinical context has mandated imaging modalities consonant with hospital-based capacities. To this end, we are exploring methods for imaging of human clinical gene therapy and virotherapy approaches for cancer. Data acquired via these imaging modalities can provide critical surrogate endpoints indicative of the biologic behavior of adenovirus agents in the human clinical contexts. Such imaging-based data is critical for the advancement of vector design modifications to improve therapeutic outcomes.

\section{6}

Metabolic imaging in cancer: what should we look for? R. DeBerardinis; Children's Medical Center Research Institute, UT Southwestern Medical Center, Dallas, TX

Changes in nutrient metabolism are hallmarks of malignant transformation, and are a potential source of therapeutic targets and diagnostic biomarkers. We are interested in understanding which metabolic characteristics differentiate tumor tissue from normal, surrounding tissue, and in developing a repertoire of techniques to monitor these features in vivo. We use stable isotope tracing and other methods to identify and quantify metabolic fluxes in cancer cells. Applying these same techniques to tumorbearing mice and human cancer patients has provided a unique view of tumor metabolism in vivo. By infusing mice and humans with ${ }^{13} \mathrm{C}$-glucose, then extracting metabolites and analyzing for ${ }^{13} \mathrm{C}$ enrichment, we determined that human gliomas oxidize glucose in the tricarboxylic acid (TCA) cycle, in addition to using glucose carbon to supply a number of other pathways, including lactate and nonessential amino acid synthesis. The complexity of metabolism in these tumors has stimulated the development of additional approaches to image informative aspects of tumor metabolism in humans and mice. These approaches include proton magnetic resonance spectroscopy to quantify glutamine, glutamate, 2-hydroxyglutarate, and other metabolites; nanoparticles tuned to activate a fluorescent signal in the acidic extracellular microenvironment of glycolytic tumors; and dynamic nuclear polarization (hyperpolarization) of ${ }^{13} \mathrm{C}$-labeled metabolites to detect specific enzyme-catalyzed reactions in vivo. We are currently attempting to combine the speed and sensitivity of ${ }^{13} \mathrm{C}$ hyperpolarization with the detail of conventional ${ }^{13} \mathrm{C}$ isotopomer analysis to produce a quantitative assessment of several biologically relevant metabolic fluxes from a single sample of tissue.

\section{7}

Assessing and monitoring therapeutic response of breast cancer and cervical cancer with FDG-PET. F. Dehdashti; Washington University, St. Louis, MO

Detection of biological changes as a result of therapy is the most sensitive method for early assessment of response to therapy. In solid tumors, the conventional approach for assessing response to therapy is based on the change in tumor size, as evaluated by physical examination or conventional imaging methods. However, change in tumor size may not occur until several courses of therapy are given and while a decrease in tumor size typically indicates a favorable response, little or no change in tumor size is not necessarily indicative of a poor response to therapy. Early assessment of the effectiveness of therapy is crucial in identifying nonresponders in order to avoid unnecessary cost and morbidity of ineffective therapy and to prevent progression of disease as a result of such therapies. Positron emission tomography (PET), a functional imaging method, offers the ability to detect early changes in tumor metabolism that are predictive of responsiveness to therapy. While many different PET radiopharmaceuticals can be used to assess tumor biology and evaluate the effectiveness of therapy, the most widely used is $\left[{ }^{18} \mathrm{~F}\right]$ fluoro-2-deoxy-D-glucose (FDG). More recently, the updated RECIST guideline (version 1.1) has included FDG-PET and stated that FDG-PET can be used to complement CT to assess disease progression. It also suggested using FDG-PET to distinguish residual disease from normal (scar) tissue when it is difficult to make the distinction by conventional imaging. Breast Cancer: The choice of systemic therapy in breast cancer is based on the presence of receptors; estrogen-receptor positive (ER+) and/or progesterone-receptor positive $(\mathrm{PgR}+)$ tumors are more likely to respond to endocrine therapy than are receptor-negative tumors. Thus, receptor-negative tumors are typically treated with chemotherapy. Patients with HER2-positive tumors are typically treated with trastuzumab-based therapy. Early assessment/ prediction of response to systemic therapy is difficult clinically and radiologically, as described above. FDG-PET has shown promise in assessment and prediction of responsiveness to both endocrine and chemotherapy of breast cancer. Typically, marked decrease in tumor FDG uptake has been associated with positive response to therapy. Changes in FDG uptake can be assessed as early as after one cycle of therapy. Unfortunately, there is no standardized protocol with an SUV cut-off value that is generally accepted to implicate a response. Similarly, FDG-PET can be used to assess and monitor response to endocrine therapy. Metabolic flare, defined as an increase in tumor FDG uptake early after therapy with estradiol or an antiestrogen agent with initial agonist effect, has been shown to be effective in distinguishing responders from nonresponders. Cervical Cancer: Response assessment in patients treated for cervical cancer based on clinical evaluation is suboptimal. Current recommendations include symptom evaluation and physical examination, which are often followed by serial pelvic examinations and cervical cytology, typically beginning 3 months after the completion of therapy. However, this strategy does not address response early after therapy or early detection of disease recurrence. Moreover, screening Pap smear is of limited utility in the posttherapy setting because of radiation-related pathological effects. Recently, FDG-PET approximately 3 months after therapy has been shown to be predictive of response and long-term outcome. Patients with complete response have significantly better survival compared with partial responders, and patients with partial response have significantly better survival compared with those who developed progression of disease. A recent study of gene expression profiling has been correlated with metabolic response. This study showed that patients with incomplete metabolic response have activation of the $\mathrm{PI} 3 \mathrm{~K} / \mathrm{Akt}$ pathway. Thus, future therapies that target inhibition of PI3K/Akt may improve response to chemoradiation.

\section{8}

FLT-PET imaging for early identification of antigen-specific immune responses in cancer patients. E. Aarntzen, M. Srinivas, C. Figdor, W. Oyen, I.J.M. DeVries; Radboud University Medical Centre, Nijmegen, The Netherlands

Current biomarkers are unable to adequately predict vaccine-induced immune protection in humans with infectious disease or cancer. However, timely and adequate assessment of antigen-specific immune responses is critical for successful vaccine development. Therefore, we have developed a method for the direct assessment of immune responses in vivo in a clinical setting. Melanoma patients with lymph node (LN) metastases received dendritic cell (DC) vaccine therapy, injected intranodally, followed by $\left[{ }^{18} \mathrm{~F}\right]-$ labeled $3^{\prime}$-fluoro-3'-deoxy-thymidine $\left(\left[{ }^{18} \mathrm{~F}\right] \mathrm{FLT}\right) \mathrm{PET}$ at varying time points after vaccination. Control LNs received saline or DCs without antigen. De novo immune responses were readily visualized in treated LNs early after the prime vaccination, and these signals persisted for up to $3 \mathrm{wk}$. This selective $\left[{ }^{18} \mathrm{~F}\right]$ FLT uptake was markedly absent in control LNs, although tracer uptake in treated LNs increased profoundly with as little as $4.5 \times 10^{5}$ DCs. Immunohistochemical staining confirmed injected DC dispersion to T-cell areas and resultant activation of CD4(+) and CD8(+) T cells. The level of LN tracer uptake significantly correlates to the level of circulating antigen-specific IgG antibodies and antigen-specific proliferation of $\mathrm{T}$ cells in peripheral blood. Furthermore, this correlation was not observed with $\left[{ }^{18} \mathrm{~F}\right]$-labeled fluoro-2deoxy-2-D-glucose. Therefore, $\left[{ }^{18} \mathrm{~F}\right] \mathrm{FLT}$ PET offers a sensitive tool to study the kinetics, localization, and involvement of lymphocyte subsets in response to vaccination. This technique allows for early discrimination of 
responding from non-responding patients in anti-cancer vaccination and aids physicians in individualized decision-making.

\section{9}

Imaging contributions of the microenvironment to chemoresistance. E. Nakasone, H. Askautrud, T. Kees, J. Park, J. Park, M. Fein, M. Egeblad; Cold Spring Harbor Laboratory, Cold Spring Harbor, NY

It is well appreciated that factors intrinsic to the cancer cells, such as specific mutations, regulate response to chemotherapy. However, there is limited knowledge on the dynamics of cancer cell death in response to therapy in the naturally developing tumor microenvironment. Using intravital microscopy (microscopy in live mice) of tumors, we show that factors within the microenvironment, extrinsic to the cancer cells, support the development of chemoresistance by regulating drug distribution and the inflammatory response. Specifically, intravital microscopy of chemotherapytreated mouse mammary carcinomas allowed us to follow drug distribution, cell death, and tumor-stroma interactions. We observed associations between vascular leakage and response to the chemotherapeutic drug doxorubicin, including improved response in matrix metalloproteinase- 9 null mice that had increased vascular leakage. Furthermore, we observed CCR2-dependent infiltration of myeloid cells after treatment and that Ccr2 null host mice responded better to treatment with doxorubicin or cisplatin. These data show that the microenvironment contributes critically to drug response via regulation of vascular permeability and innate immune cell infiltration. These results have clinical implications, as myeloid cell infiltration is increased in human breast tumors after chemotherapy and the cellular composition of the immune infiltrate is a strong predictor of overall survival. Our data further suggest that the response to classical chemotherapeutic drugs can be improved by changing the tumor microenvironment with agents that modify matrix metalloproteinase activity and chemokine signaling. Thus, intravital imaging can be used to gain insights into drug responses in situ.

\section{0}

Imaging the role of the immune system during oncogene-induced tumorigenesis. D.W. Felsher; Stanford University, Stanford, CA

The targeted inactivation of oncogenes can elicit oncogene addiction. Previously, my laboratory has identified both cell autonomous/tumor intrinsic and cell non-autonomous host-dependent mechanisms of oncogene addiction including: proliferative arrest, apoptosis, differentiation and senescence of tumor cells and the recruitment of immune effectors and the shutdown of angiogenesis in the host. Our most recent work suggests a critical noncanonical role for host immune cells in regulating many of these mechanisms. To directly interrogate the role of the host immune system in modulating the local tumor microenvironment as a mechanism of oncogene addiction, we have used multiple ex vivo and in situ imaging approaches including bioluminescence imaging, whole body fluorescent imaging, and microPET imaging. We have been able to measure the tumor growth and regression kinetics, apoptosis and proliferation changes upon oncogene inactivation with specific apoptosis and proliferation probes. Moreover, through an intravital microscopic imaging (IVM) strategy, we can monitor the dynamic processes and cell-cell interactions at a high resolution in the same tumor over time. We have applied IVM to interrogate the spatiotemporal relationships between tumor cell-intrinsic and hostdependent changes and identify the specific role of immune effectors in regulating oncogene addiction. Through a novel PET-based approach we are able to monitor changes in proliferation and apoptosis in tumor cells upon oncogene inactivation. We have used these probes to perform a detailed analysis of the kinetics of changes in cellular proliferation and apoptosis. Our work suggests a complex role for host immune cell homing to the site of the tumor before and after oncogene inactivation as well as changes in angiogenesis. Our results provide the first in situ examination of the mechanisms of oncogene addiction.

\section{1}

Radiomics: informing cancer heterogeneity. R.J. Gillies; H. Lee Moffitt Cancer Center, Tampa, FL
It is becoming increasingly appreciated that malignant cancers can be characterized by genetic instability within highly selective local microenvironments. This combination promotes somatic evolution and the emergences of clades of cells in spatially explicit micro-habitats. Malignancy can be defined by these habitats, which increase the probability that cancers will develop therapy resistant phenotypes (1). Heterogeneity across cancers has been known at the nuclear level for years (2) and is recognized as a strong predictor of poor prognosis. This increases the probability of therapy resistance. For most advanced cancers and most patients, response to therapy is fleeting, owing to the inevitable evolution and proliferation of a resistant population. Induction of genomic alterations and localized selection by heritable and/or environmental factors will result in phenotypic heterogeneity. Heterogeneity can be viewed radiographically, wherein a non-uniform pattern of enhancement or attenuation can be associated with poor outcome $(3,4)$. In order to systematically address this issue, we have created a database structure that can be populated with images, as well as quantitative image feature data (e.g. texture, shape, density features) that can be mined in combination with patient outcomes and genetic data from biopsies. This is to allow real-time data analyses and association of features with prognostic, diagnostic and predictive models (5). The goal of radiomics is to convert images to mineable data, with high fidelity and high throughput. The radiomics enterprise can be divided into five processes with definable inputs and outputs, each with its own challenges that need to be overcome: (i) image acquisition and reconstruction; (ii) image segmentation and rendering; (iii) feature extraction and feature qualification; (iv) databases and data sharing; and (v) ad hoc informatics analyses (6). Each of these steps must be developed de novo and, as such, poses discrete challenges that have to be met. Even though this field is in its infancy, meaningful classifier models have been generated in detecting and diagnosing a number of cancer subtypes. Foremost among these are lung cancers for whom large amounts of PET and CT data are available. References: 1. Gillies RJ, Verduzco D, Gatenby RA. Evolutionary dynamics of carcinogenesis and why targeted therapy does not work. Nature Reviews. 2012;12(7):487-493. 2. Winge O. Zytologische untersuchungen uber die natur maligner tumoren. II. Teerkarzinome bei mausen. Z Zellforsch Mikrosk Anat. January 22, 1930;10:683-735. 3. Iwamoto FM, Abrey LE, Beal K, et al. Patterns of relapse and prognosis after bevacizumab failure in recurrent glioblastoma. Neurology. 2009;73:1200-1206. 4. Kawata Y, Niki N, Ohmatsu H, et al. Quantitative classification based on CT histogram analysis of non-small cell lung cancer: correlation with histopathological characteristics and recurrencefree survival. Medical Physics. Feb 2012;39(2):988. 5. Lambin P, RiosVelazquez E, Leijenaar R, et al. Radiomics: extracting more information from medical images using advanced feature analysis. Eur $\mathrm{J}$ Cancer. 2012;doi:10.1016/j.ejca.2011.11.036. 6. Kumar V, Gu Y, Basu S, et al. Radioomics: the process and the challenges. Magnetic Resonance Imaging. 2012; in press.

\section{2}

MRI to assess breast cancer response to treatment: experience in the I-SPY/ACRIN trials. N. Hylton; University of California, San Francisco, $\mathrm{CA}$

Contrast-enhanced MRI is now used routinely for the detection and diagnosis of breast cancer. Compared to mammography and ultrasound, MRI has particular advantages for demonstrating the anatomic extent of cancer in the breast, essential information for disease staging and treatment planning. MRI can also provide quantitative in vivo information about the state of disease and can be used to measure changes associated with therapy. Quantitative MRI techniques such as dynamic contrast-enhanced (DCE)MRI and diffusion-weighted MRI (DWI) have been applied to measure breast tumor changes associated with response to neoadjuvant treatment. These techniques add functional information about breast cancer by sensitizing the MRI signal to tumor vascularity, known to be associated with tumor grade and aggressiveness, and water diffusion, a property affected by cell density that can reflect tumor growth as well as cell death from cytotoxic treatment. These methods are being tested in the I-SPY TRIAL integrating biomarkers and imaging to maximize effectiveness of neoadjuvant treatment for patients with locally-advanced breast cancer. The first phase of the ISPY TRIAL, I-SPY-1, was conducted as an observational study with patients on standard neoadjuvant treatment enrolling on two correlative science trials 
designed to evaluate imaging and tissue biomarkers (ACRIN 6657 and CALGB 150007, respectively). ACRIN 6657 is designed to test whether MRI measurements of tumor response can be used to predict recurrencefree survival, and whether MRI measurements after 1 cycle of treatment can identify those patients whose tumors are non-responsive after treatment. I-SPY-1 opened in May 2002 and completed accrual of 237 patients in March 2006; 5-year follow-up was completed in 2011. In initial results, tumor volume measured by MRI was shown to be a sensitive measure of tumor response to treatment and was able to provide early prediction of response after only one cycle of chemotherapy. The continuing trial, I-SPY2 , is a breast cancer treatment study and uses an adaptive phase 2 design to evaluate targeted drugs in the setting of neoadjuvant therapy. As part of the adaptive design of ISPY-2, change in tumor volume measured by DCE-MRI at serial time points during chemotherapy is used to adjust the randomization schema as the trial proceeds. I-SPY-2 opened in March 2010 and is currently open at 20 sites, with 2 additional sites anticipated. A concurrent imaging study, ACRIN 6698, was developed to evaluate diffusion-weighted imaging (DWI) for breast tumor response assessment, and opened in August 2012. Results from I-SPY 1 and the ongoing I-SPY 2 trial will be discussed as well as the experience implementing standardized MRI protocols in the multi-center setting.

Imaging signaling pathways and tumor-stromal interactions in cancer metastasis. Y. Kang; Princeton University, Princeton, NJ

Metastasis represents the most devastating stage of cancer progression. In particular, bone metastasis is a frequent occurrence in breast cancer, affecting more than $70 \%$ of late stage cancer patients. Discovering bone metastasis genes that are clinically relevant and functionally important is critical for the development of novel therapeutics for high-risk breast cancer patients. It is important to understand the functional dynamics of novel metastasis proteins in mediating tumor-stromal interactions essential for the formation of osteolytic bone metastasis. We have developed methods to image real-time signaling pathways activities during the development of metastatic diseases. Using in vivo imaging technology, we showed that TGF $\beta$ is released from bone matrix upon bone destruction, and signals to breast cancer to further enhance their malignancy, in part through increasing Jagged1/Notch signaling from tumor to stroma. While blocking TGF $\beta$ signaling prevents or reduces the development of bone metastasis at the early stage, well established bone metastases are much less sensitive to TGF $\beta$ inhibitor treatments. These studies suggest the therapeutic benefit of intercepting the TGF $\beta$-dependent vicious cycle in bone metastasis, particularly when treatments are applied in the adjuvant setting or on early bone lesions. In breast cancer patients, disseminated tumor cells from the primary site can maintain in a dormant state in the secondary organ for a long period before starting to grow into life-threatening metastasis. However, the molecular understanding of this process is still very limited. We recently discovered that Vascular Cell Adhesion Molecule-1 (VCAM1) is essential for the conversion from dormant bone micrometastases to macrometastasis in a xenograft mouse model of breast cancer bone metastasis. Using an ex vivo live imaging model of bone metastasis at the single cell level, we showed that VCAM1 mediates metastasis transition by promoting recruitment of preosteoclasts via binding to the receptor integrin VLA-4 expressed on osteoclast precursors. We successfully blocked the initiation and progression of bone metastasis using purified monoclonal antibodies for VCAM1 or VLA-4. These studies demonstrated the power of combining in vivo imaging with mechanistic studies of metastasis proteins to develop novel treatment approach to prevent or reduce metastatic disease.

\section{4}

Targeted microbubbles: ultrasound contrast agents for molecular imaging. Z. Du ${ }^{1}$, S. Unnikrishnan ${ }^{1}$, D. Smith ${ }^{2}$, J.J. Rychak ${ }^{2}$, A.L. Klibanov'; ${ }^{1}$ University of Virginia, Charlottesville, VA, ${ }^{2}$ Targeson, Inc., San Diego, CA

Urgent need exists to broaden clinical use of molecular imaging and obtain crucial information on molecular markers of disease, especially in oncology. This information should be acquired safely, quickly, non-invasively and inexpensively. Ultrasound molecular imaging can get the required data for a subset of targets located at the tumor vascular endothelium in soft tissues. Prostate and breast cancer imaging should be considered as first clinically suitable targets. Fluorocarbon gas-filled microbubbles (mean size $\sim 2 \mu \mathrm{m}$ ) are stabilized with a thin shell (typically, $\sim 2 \mathrm{~nm}$ lipid monolayer). Ultrasound contrast formulations are biocompatible: they are used as blood pool agents worldwide and have been injected in millions of patients. Targeting ligands can be linked to bubbles noncovalently, via avidin-biotin coupling, for simplified early stage preclinical studies. Covalent attachment of targeting ligands to the bubble shell is necessary for ultrasound contrast formulations to be considered for clinical development. Use of a spacer arm (flexible PEG polymer or larger extender structures on the microbubble surface) improves targeting efficacy and allows conversion of transient adhesion events to permanent retention of microbubbles on the target surface. A wide variety of targeting ligands has been successfully attached to the bubbles, including antibodies, other proteins, peptides, carbohydrates $(\sim 100,000$ ligand molecules per one microbubble are placed typically). Combination of ligands on the microbubble surface is possible. These ligand-carrying microbubble particles can freely circulate in the bloodstream and accumulate in the areas where respective receptors are upregulated: VCAM-1, ICAM-1, alphaVbeta3, VEGFR2, E- and P-selectins. Clinical diagnostic ultrasound can visualize single microbubble contrast particles, with sub-picogram mass each. It is therefore possible to delineate the areas with high level of endothelial expression of the disease markers, which results in selective adhesion of microbubbles and their detection by ultrasound imaging. A typical molecular imaging scenario consists of administering an intravenous bolus of targeted microbubble construct, followed by nondestructive realtime ultrasound imaging of tissue perfusion in the region of interest. Circulating ultrasound contrast clears from the bloodstream in 10-15 min: fluorocarbon gas leaves bubble shell and is exhaled via lungs. Ultrasound imaging of the targeted microbubbles in the tumor vasculature is then performed to delineate the location of the nodes and guide biopsy and/or therapeutic interventions. After that, targeted microbubbles can be "wiped" from the tissue by increasing the transmitted acoustic power of the imaging system probe, and then a formulation directed towards another receptor can be administered. The amount of administered ultrasound contrast needed for molecular imaging is quite low: only a small fraction of microbubbles reaches the target, yet excellent detection sensitivity makes it possible to inject sub-mg dose. A choice of fast-adhering ligands aids targeting in highshear flow conditions. Acoustic radiation force application improves targeting efficacy - it allows more efficient probing of vessel wall by targeted bubbles and may thus help reduce the required contrast dose well under microdosing limits. A more complex task now under investigation is to use molecular ultrasound imaging to obtain quantitative receptor information. Indeed, the increase of disease marker surface density on the target vascular endothelium results in the increase of targeted bubble accumulation. However, in vitro flow chamber calibration curve may not find immediate applicability in vivo: presence of red blood cells strongly influences targeted microbubble adhesion, and more detailed studies are needed. Overall, molecular ultrasound imaging with targeted microbubble contrast is rapidly advancing from preclinical testing towards widespread application in the clinic.

\section{5}

Imaging glutaminolysis in cancer. H.F. Kung ${ }^{1}$, K. Ploess $1^{1}$, B.P. Lieberman $^{1}$, L. Wang ${ }^{1}$, W. Qu ${ }^{1}$, J.S. Lewis ${ }^{2}$, M. Dunphy ${ }^{2}$, C.B. Thompson ${ }^{2}$; ${ }^{1}$ University of Pennsylvania, Philadelphia, PA, ${ }^{2}$ Memorial Sloan-Kettering Cancer Center, New York, NY

Position emission tomography (PET) with 2-[ $\left.{ }^{18} \mathrm{~F}\right]$ fluoro-2-deoxy-glucose (FDG) is a common imaging procedure for mapping cellular glucose metabolism. The uptake of FDG in tumors with higher rates of glycolysis leads to high signal to noise ratios. Enhanced anatomical identification with PET/CT has significantly improved the identification of tumor with a higher rate of glycolysis. Despite the tremendous promise of using FDGPET to detect and monitor tumor metabolism, a significant portion of malignant tumors in cancer patients $(\sim 30 \%)$ is not FDG-positive. Many indolent tumors are FDG-negative; the tumors may have switched their energy source from glucose to glutamine, thus escaping detection. To improve cancer diagnosis we have prepared and tested a series of novel fluorinated glutamine derivatives mimicking glutamine, which is fueling 
the tumor growth. Four different isomers of $\left[{ }^{18} \mathrm{~F}\right] 4$-fluoro-glutamine $(2 \mathrm{~S}, 4 \mathrm{~S} ; 2 \mathrm{~S}, 4 \mathrm{R} ; 2 \mathrm{R}, 4 \mathrm{~S} ; 2 \mathrm{R}, 4 \mathrm{R})$ were tested by in vitro cell uptake assays and in vivo PET imaging in rats. Only the $2 \mathrm{~S}$ isomers, the naturally occurring glutamine configuration, showed a significant glutaminespecific uptake. Cell uptake in SF188 and 9L glioblastoma tumor cells displayed a higher rate of uptake, which was higher than that of FDG. Dynamic small animal PET imaging studies of $\left[{ }^{18} \mathrm{~F}\right](2 \mathrm{~S}, 4 \mathrm{R}) 4$-fluoroglutamine were conducted in 9L tumor-cell-xenografts produced in F344 rats. Biodistribution and PET imaging studies showed that $\left[{ }^{18} \mathrm{~F}\right](2 \mathrm{~S}, 4 \mathrm{R}) 4$ fluoro-glutamine localized in tumors with a higher uptake than in surrounding muscle and liver tissues. In addition, at 30 and 60 minutes after an iv injection into tumor bearing rats, $\left[{ }^{18} \mathrm{~F}\right](2 \mathrm{~S}, 4 \mathrm{R}) 4$-fluoroglutamine displayed a high incorporation (30\% and $70 \%$, respectively) into macromolecules suggesting a rapid intracellular metabolism. Preliminary results of animal toxicity and radiodosimetry studies suggest that $\left[{ }^{18} \mathrm{~F}\right](2 \mathrm{~S}, 4 \mathrm{R}) 4-$ fluoro-glutamine is safe for human application. This imaging agent may be useful for detecting and monitoring tumor metabolism based on glutamine utilization.

\section{6}

Cancerous stem cells with genomic instability: the best vehicle with the best engine for cancer. J. Chandler, E. Lagasse; McGowan Institute for Regenerative Medicine, University of Pittsburgh School of Medicine, Pittsburgh, PA

Human cancers have been found to include transformed stem cells that may drive cancer progression to metastasis. In metastatic colon cancer, we identified tumor cells with all of the critical properties expected of stem cells, including self-renewal and lineage maturation. Additionally, these cancer stem cells initiated tumors in mice that closely resemble human cancer. Karyotypic analyses performed on parental and clonallyderived tumor cells expressed many similar and some different chromosomal aberrations, suggesting the presence of chromosomal instability in the cancer stem cells. Thus, the model proposed here for cancer and its metastatic progression includes both the features of the hierarchy model with cancerous stem cells and the stochastic model, driven by the observation of chromosomal instability, to explain tumor cell heterogeneity and possibly the origin of metastases. Interestingly, cancer of the intestine is almost exclusively found in the large intestine, although the intestine is often thought to be one organ, with one stem cell. Due to the differences found between the small and large intestine in cancer incidence rate, we hypothesize that there are intrinsic differences in resident stem cells in the small versus large intestine, leading to the increased frequency of human colon cancer. Preliminary studies indicate shared and disparate characteristics among the stem cells of normal small and large intestine and colon cancer stem cells, suggesting that colon cancer could be considered a stem cell disease whose treatment requires targeting colon cancerous stem cells to eliminate the chance for recurrence and metastases.

\section{7}

Image-guided siRNA and miRNA therapies. A. Moore; Massachusetts General Hospital, Boston, MA

RNA interference is an innate cellular mechanism for post-transcriptional regulation of gene expression in which double-stranded ribonucleic acid inhibits the expression of genes with complementary nucleotide sequences. Its potential for tumor therapy is indisputable, considering that one can use this mechanism to silence virtually any gene, including genes implicated in tumorigenesis, with single-nucleotide specificity. Major obstacles in applying RNA interference in vivo are presented by the short circulation half-life of the siRNA molecule, its vulnerability to degradation by nucleases (elimination half-life 2-6 min), and the need to translocate the siRNA into the cytosol, where the RNA interference process takes place. An essential element in the development and optimization of a siRNA delivery method is the ability to measure the bioavailability and functionality of the siRNA molecule after administration into the body. Noninvasive imaging provides the necessary set of tools to accomplish this in authentic physiologic environments and across time. To facilitate siRNA delivery to tumors and enable in vivo imaging of the delivery, magnetic nanoparticles, which allowed for visualization by magnetic resonance imaging, have been employed. MicroRNAs are a class of post-transcriptional regulators that have been implicated in various cell functions including metastatic potential. Several microRNAs that mediate the process of tumor cell migration and tissue invasion have been identified. In our studies we focused on targeting miR-10b implicated in breast cancer metastasis using magnetic nanoparticles decorated with locked nucleic acid oligonucleotides as a therapeutic moiety. These nanoparticles have an inherent capacity for noninvasive imaging and obtaining semi-quantitative information about nanoparticle bioavailability in target tissues. This information could be invaluable in a clinical setting for determining the need for re-treatment on a patient-by-patient basis, opening up the possibility for designing individualized therapeutic regimens. This presentation will focus on molecular imaging techniques that can be used for noninvasive monitoring of siRNA and microRNA therapies in cancer.

\section{8}

Imaging the cancer stem cell marker AC133. G. Niedermann; University Clinics Freiburg, Freiburg, Germany

Tumor stem cells are highly undifferentiated tumor cells with characteristics similar to normal stem cells. These characteristics include extensive self-renewal and a more or less impaired capacity for multilineage differentiation. Based on that, tumor stem cells are thought to drive tumor growth but also to generate more differentiated tumor cells that are less tumorigenic or non-tumorigenic. In malignant tumors that follow the stem cell model, elimination of tumor stem cells would be particularly crucial for successful tumor treatment. However, tumor stem cells are usually more resistant to conventional radio- and chemotherapy than more differentiated tumor cells. Moreover, there is evidence that tumor stem cells are also important players in tumor invasion and metastasis. Given all these very important potential roles in tumor propagation and progression as well as in treatment resistance, approaches to image tumor stem cells noninvasively may be very useful for monitoring treatment responses and for early detection of relapsed tumors. AC133, a glycosylation-dependent epitope of CD133, is one of the best characterized tumor stem cell markers, and AC133+ tumor stem cells have been described for many tumor entities. Using the AC133 antibody, we have developed methods to image AC133+ tumor stem cells noninvasively by near-infrared fluorescence molecular tomography and positron emission tomography in immunodeficient mice bearing xenografts of human tumors. Noninvasive detection of tumor-associated AC133 could also guide molecularly targeted therapies aimed at eliminating tumor stem cells. Along this line, I will finally present data on the elimination of $\mathrm{AC} 133+$ tumor stem cells with a novel recombinant, therapeutic antibody which we have generated. Since the normal stem cell regions in the adult brain are largely $\mathrm{AC} 133$-negative, we pay particular attention to developing novel options for the local treatment of brain tumors, especially for the very aggressive glioblastoma multiforme which still is invariably fatal. Results obtained using immunodeficient mouse models and orthotopically growing glioblastomas will be presented.

\section{9}

Predictive computational models of cancer metabolism at the genome scale. J. Papin; University of Virginia, Charlottesville, VA

The genomics revolution has led to the generation of an enormous amount of data on the composition, regulation, and physiology of cellular networks. There is a need to integrate this information into a computational framework so that testable predictions can be made with an accounting of the complexity inherent in cellular systems. Recent advances on the integration of expression profiling data with metabolic and transcriptional regulatory network reconstructions will be presented. The resultant genome-scale models have been used to make experimentally testable predictions, and novel methods to identify ideal drug targets will be presented. The potential role of molecular imaging to facilitate the reconstruction and validation of these networks will also be discussed. Systems biology approaches hold the promise of revolutionizing drug discovery efforts and tackling key challenges in cancer. 
and transmit them throughout the cell to elicit appropriate biological

Optical imaging at the nanometer scale: studies of molecular structures and interactions. A. Pertsinidis; Memorial Sloan-Kettering Cancer Center, New York, NY

Objectives: Fluorescence imaging allows probing intra-cellular processes with pristine molecular specificity, under native, physiological conditions. Recently, a new class of techniques has emerged that bypass the diffraction limit of conventional microscopy, achieving a remarkable increasetypically up to 10 -fold-in spatial resolution. Several proof-of-concept experiments in systems with well-defined spatial organization (e.g. cell cytoskeleton) have demonstrated the higher clarity that is possible with the new capabilities. Despite these notable advances however, the ultimate potential of such techniques in deciphering unknown molecular structures and interactions has yet to be fully realized. Methods: The essence of subdiffraction imaging methods is reconstructing an image based on highlyaccurate measurements of the positions of all the fluorescent probes labeling the structure of interest. The effective resolution is thus given by the localization uncertainty, which, in principle, should be able to reach down to arbitrarily small scales, limited only by the signal and background in the detection of single fluorescent molecules. We have employed a variety of approaches to reduce/eliminate the various sources of systematic errors that had prevented previous implementations from reaching the ultimate limit, including drift and vibration of the measurement apparatus, imperfections in the optics and detector, asymmetry in the fluorescence emission and the photostability of the fluorescent dye. Results: We have demonstrated a reduction in the extraneous sources of errors down to the sub-nanometer level, or about $1 / 10$ th the size of a typical protein molecule. This first viable strategy to obtain molecular-scale structural information based on singlemolecule imaging techniques allowed deciphering the relative arrangement of components in a protein complex formed by the cell adhesion molecule E-cadherin. We have also extended the same principles in visualizing cellular ultra-structure in situ, by imaging multiple closely-spaced identical fluorophores that are randomly switched on in time. With the new capabilities, we delineated the correlations of two different distinct intracellular species at nanometer scales, visualizing the association of SNARE and SM-proteins, key players in protein trafficking and membrane fusion reactions in the cell. Finally, we applied the multi-color nanometer co-localization assay to detect the assembly of components of the RNA Polymerase II transcriptional machinery, a complex reconstituted system of $>40$ polypeptides with distinct chemical properties and functionalities, and the accurate production of individual mRNAs, at the single-molecule level. Conclusions: Our results provide a framework for analyzing molecularscale arrangements with sub-diffraction fluorescence imaging and for investigating molecular interactions between sub-cellular systems, in situ. The ultra-high-resolution, multiplex detection capabilities afforded by these techniques create new possibilities for investigating complex biomolecular processes in cells, including signal transduction, cell-cell communication and gene expression regulation, with molecular precision. Acknowledgments: The work reported was partially supported by the Louis V. Gerstner, Jr., Young Investigators Fund and the National Institute of General Medical Sciences of the National Institutes of Health under Award Number DP2GM105443.

\section{1}

Cancer genomic and systems heterogeneity: characterization and challenges. D. Piwnica-Worms; Washington University Medical School, St. Louis, MO

Large-scale sequencing analyses of solid cancers have identified extensive genetic inter-tumor and intra-tumor heterogeneity, which likely contribute to treatment failure and drug resistance. For example, recent unbiased whole genome sequencing approaches have highlighted the complexity of the mutational landscape of ER+ luminal breast cancers. In 46 breast tumors, 82,000 total somatic mutations and 2,000 tier 1 mutations were identified While pathway signatures enabled the evaluation of mutations with low recurrence frequency where statistical comparisons conventionally were underpowered, this massive heterogeneity presents an enormous challenge for therapy and prognostic assessment of cancer. Furthermore, cells have evolved complex molecular networks to sense cues from the environment responses. These signaling pathways require certain elemental properties, such as sensitivity, reversibility, a capacity to be regulated, and robustness, that are crucial to reliably maintaining the organization and function of cells within organisms. These molecular networks comprise sets of recurring regulation patterns, manifest as network motifs, which link together in a variety of combinations to create a web of connectivity within a given signaling pathway or between multiple cascades. Using a $\kappa \mathrm{B}_{5} \rightarrow \mathrm{I} \kappa \mathrm{B} \alpha$-FLuc bioluminescent reporter as one example, we rigorously evaluated the dynamics of $\mathrm{I} \kappa \mathrm{B} \alpha$ degradation and subsequent $\mathrm{NF} \kappa \mathrm{B}$ transcriptional activity in response to diverse modes of TNF $\alpha$ stimulation in isogenic HepG2 tumor cells. Tremendous heterogeneity in the transcriptional amplitudes of responding cells was observed. Thus, systems heterogeneity was observed even in isogenic cells, further adding to the complexity of cellular responses and their associated signaling networks. One must conclude that inter-tumor and intra-tumor heterogeneity at the level of cancer genes and systems responses may have important consequences for targeted molecular imaging strategies that commonly lack spatial and temporal resolution in their depiction of tumor phenotypes.

\section{2}

Imaging of oncolytic viral therapies of cancer. S. Thorne; University of Pittsburgh, Pittsburgh, PA

Oncolytic virotherapies are cancer therapies based on genetically engineered viruses designed to selectively replicate in cancer cells. Several oncolytic viruses, such as those based on vaccinia virus, have demonstrated highly promising Phase II clinical results and have entered randomized testing. However the replicative capacity of these viruses makes dosing complex, and interpretation of clinical results, especially for non-responders, can be difficult. For this reason it has become evident that in vivo pre-clinical and clinical imaging of viral gene expression is critical for development of the therapeutic (including understanding of systemic delivery capacity, therapeutic index and persistence), while the capacity to image gene expression in any approved products would also considerably enhance the clinical application of these agents (through determination of likely success of the therapy prior to any tumor volume endpoint measurements). The capacity of vaccinia virus to incorporate multiple transgenes allows for the easy insertion of reporter genes for imaging. The benefits and limitations of different reporters and imaging modalities for use in oncolytic vaccinia therapies will be discussed. These will include the sodium-iodide symporter and somatostatin receptor gene for radiological imaging, ferritin genes for MRI and several optical reporters for use in pre-clinical development. In addition the possible future application of novel reporters capable of multimodality imaging will be discussed. These genes and imaging approaches might also be applied to other gene therapy or immune cell therapies and the capacity to image multiple different reporters within the same host or patient is likely to be an important future consideration as combinations of biological therapies are examined. Furthermore, the potential use of radioisotopes for additional therapeutic benefit and the use of oncolytic vaccinia as a theranostic agent for detection of metastases and identification of draining lymph nodes will be discussed.

\section{3}

Expediting translation of optical imaging into the clinic. G.M. van Dam; University Medical Center, Groningen, The Netherlands

In recent years, significant progress has been made in both optical imaging systems and fluorescent contrast agents for clinical applications in oncology. NIRF imaging with a free-floating imaging device mounted on the ceiling of the operating theatre or on a microscope articulating arm during surgery for cancer will enable visualization of tumor delineation, locoregional metastases, remnant disease as well as e.g. tumor-containing lymph nodes. Hereby, the surgeon can both detect (diagnostic) and excise (therapeutic) malignant tissue and possible residual disease at the same time. The use of NIRF optical imaging has a range of advantages. Most prominent among these is the fact that it is very safe technology, simple to operate, fast, high resolution, relatively inexpensive and makes use of non-ionizing radiation. Based on the above, it is clear that intra-operative imaging is on the verge of entering standard clinical practice for surgery. Not only the imaging system 
but also the availability of clinical grade tumor-targeted near-infrared probes is of the utmost importance for a successful introduction into clinical practice. This talk will give an overview of the current concepts and future perspectives of intraoperative fluorescence image-guided surgery using non-targeted and targeted optical contrast agents for the first-time ever in patients with ovarian cancer and the anticipated developments within the next 5 years. It will become clear that agencies like the FDA and EMA will play a major role in bringing this technology into the clinic and the necessity of standardization of methodology when applied in multinational multicenter studies.

\section{4}

Photoacoustic tomography: ultrasonically breaking through the optical diffusion limit. L. Wang; Washington University, St. Louis, MO

We develop photoacoustic imaging technologies for in vivo early-cancer detection and functional or molecular imaging by physically combining nonionizing electromagnetic and ultrasonic waves. Unlike ionizing $\mathrm{x}$-ray radiation, non-ionizing electromagnetic waves - such as optical and radio waves - pose no health hazard and reveal new contrast mechanisms. Unfortunately, electromagnetic waves in the non-ionizing spectral region do not penetrate biological tissue in straight paths as x-rays do. Consequently, high-resolution tomography based on non-ionizing electromagnetic waves alone - such as confocal microscopy, two-photon microscopy, and optical coherence tomography-is limited to superficial imaging within approximately one optical transport mean free path ( $\sim 1 \mathrm{~mm}$ in the skin) of the surface of scattering biological tissue. Ultrasonic imaging, on the contrary, provides good image resolution but has strong speckle artifacts as well as poor contrast in early-stage tumors. Ultrasound-mediated imaging modalities that combine electromagnetic and ultrasonic waves can synergistically overcome the above limitations. The hybrid modalities provide relatively deep penetration at high ultrasonic resolution and yield speckle-free images with high electromagnetic contrast. In photoacoustic computed tomography, a pulsed broad laser beam illuminates the biological tissue to generate a small but rapid temperature rise, which leads to emission of ultrasonic waves due to thermoelastic expansion. The short-wavelength pulsed ultrasonic waves are then detected by unfocused ultrasonic transducers. High-resolution tomographic images of optical contrast are then formed through image reconstruction. Endogenous optical contrast can be used to quantify the concentration of total hemoglobin, the oxygen saturation of hemoglobin, and the concentration of melanin. Melanoma and other tumors have been imaged in vivo. Exogenous optical contrast can be used to provide molecular imaging and reporter gene imaging. In photoacoustic microscopy, a pulsed laser beam is focused into the biological tissue to generate ultrasonic waves, which are then detected with a focused ultrasonic transducer to form a depth resolved 1D image. Raster scanning yields 3D high-resolution tomographic images. Super-depths beyond the optical diffusion limit have been reached with high spatial resolution. A skin image was acquired in vivo in a mouse using opticalresolution photoacoustic microscopy. The annual conference on this topic has been doubling in size approximately every three years since 2003 and has become the largest in SPIE's Photonics West as of 2009.

\section{5}

Imaging therapeutic response with PET. W.A. Weber; Universitätsklinikum Freiburg, Freiburg, Germany
Most anticancer drugs are effective only in subgroups of patients, and it is currently not feasible to predict accurately whether an individual tumor will respond and what will be the duration of response. Measuring tumor shrinkage on CT represents the current standard for response assessment, but fundamental limitations remain (e.g. interobserver variability of size measurements, limited ability to differentiate viable tumor from fibrotic tissue). CT is also limited in characterizing responses in tumors that do not decrease in size during therapy. Because the growth rate of untreated human tumors varies tremendously, an unchanged size after some weeks of therapy may represent a drug effect but may also indicate a slowly growing tumor that was not affected by the applied therapy. Molecular imaging with PET and the glucose analogue FDG has been shown to improve response assessment and now plays a key role in defining tumor response in patients with lymphoma. In solid tumors, standardized criteria for response assessment by FDG-PET (PERCIST) have recently been proposed and detailed international guidelines for quantitative PET imaging in clinical trials are available. PET based adaptive therapies are currently being evaluated in lymphomas and several solid tumors. It is expected that adaptive treatment strategies will become increasingly important in the future as more and more alternative targeted drugs are becoming available. In addition to FDG, many other imaging probes for characterizing tumor response to therapy have been developed in recent years. These include markers of amino acid transport and metabolism, multiple receptor ligands (androgen, estrogen, various $\mathrm{G}$ protein coupled receptors, integrins). The use of these promising molecular probes in clinical trials and for patient management is currently hampered by complex regulations and the lack of industrial sponsors for clinical trials.

\section{6}

Imaging cancer and the immune response with positron emission tomography. M.N. McCracken, E. Gschweng, E. Nair-Gill, J. McLaughlin, A. Cooper, M. Riedinger, D. Cheng, C. Nosala, D.B. Kohn, O.N. Witte; UCLA, Los Angeles, CA

Inert reporter gene strategies capable of being transitioned into clinical studies are needed to monitor the location of transplanted cells noninvasively. Positron emission tomography (PET) reporter genes allow visualization of cells by detection with radiolabeled probes. Previous PET reporter studies have used a viral enzyme that is immunogenic in humans leading to the clearance of reporter cells. We utilized a human deoxycytidine kinase containing three point substitutions within the active site (hdCK3mut) as a reporter gene in combination with the PET probe $\left[{ }^{18} \mathrm{~F}\right]$-L-FMAU (1-(2-deoxy-2- ${ }^{18}$ fluoro- $\beta$-L-arabinofuranosyl)-5methyluracil) to monitor models of mouse and human HSC transplantation. These substitutions in hdCK3mut expanded the substrate capacity allowing for reporter specific detection with a thymidine analog probe. There have been minimal studies investigating the long-term effects of reporter gene expression in non-transformed cells. Measurements of long-term engrafted cells (up to 32 weeks) demonstrated that hdCK 3 mut expression is maintained in vivo with no counter selection against reporter labeled cells. Reporter cells retained equivalent engraftment and differentiation capacity, being detected in all major hematopoietic lineages and tissues. This reporter gene and probe should be applicable to non-invasively monitor therapeutic cell transplants in multiple tissues. 